$$
\text { الجمهورية الجزائرية الايمقراطية الثعبية }
$$

République Algérienne Démocratique et Populaire

$$
\text { وزارة التعليم العالي والبحث العلمي }
$$

Ministère de l'Enseignement Supérieure et de la Recherche Scientifique

Université Mohamed Khider- Biskra

Faculté des Sciences et de la Technologie

Département : Génie Mécanique

Ref :
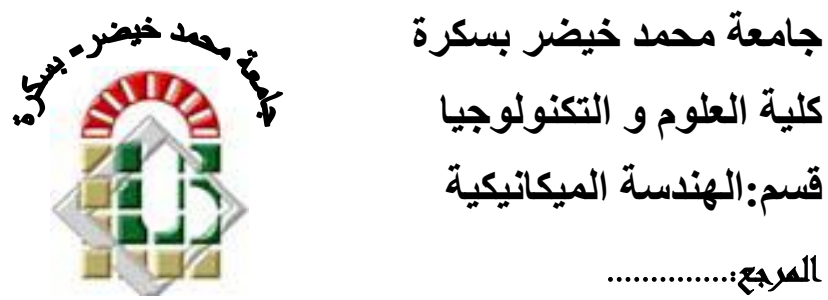

Thèse présentée en vue de l'obtention

Du diplôme de

Doctorat en science en : Génie Mécanique

Option : Génie Mécanique

\title{
Intitulé
}

\section{Congélation solaire par procédé thermochimique à partir d'une source thermique solaire basse température dans le sud Algérien.}

Présentée par :

\section{Nacer HEBBIR}

Soutenue publiquement le : 13-12-2015

\section{Devant le jury composé de :}

Dr. HADID Mohamed
Dr. CHEBBAH Mohamed-Saïd
Dr. KAJA Mahfoud
Dr. RAHMANI Ahmed

Dr. HADID Mohamed

Dr. RAHMANI Ahmed
Professeur

Maitre de Conférences ' $A$ '

Professeur

Maitre de Conférences ' $A$ '
Président Université de Biskra

Rapporteur Université de Biskra

Examinateur Université de Constantine 1

Examinateur Université d'Oum El Bouaghi 


\section{$\underline{\text { Remerciements }}$}

Je tiens à exprimer mes vifs remerciements à Monsieur Mohamed-Saïd CHEBBAH, Maître de Conférences de l'Université de Biskra, pour avoir dirigé ce travail, et pour la confiance et l'intérêt qu'il a témoigné tout au long de la réalisation de ce travail.

Mes remerciements distingués vont à Monsieur Mohamed HADID, Professeur de l'Université de Biskra, pour accepter de présider le jury.

Je remercie vivement Monsieur Mahfoud KADJA, Professeur de l'Université de Constantine 1, pour avoir accepté d'examiner ce mémoire.

Mes vifs remerciements vont aussi à monsieur Ahmed RAHMANI, Maître de Conférences de l'Université d'Oum El Bouaghi pour avoir accepté d'examiner ce travail en me faisant l'honneur de participer à ce jury.

Enfin je remercie tous ceux qui ont participé de près ou de loin à la réalisation de ce travail.

Nacer Helbir. 


\section{dédicace}

JE DÉDIE CE MODESTE TRAVAIL À:

\section{$\checkmark M A$ FEMME}

$\checkmark$ MES FILLES : AMMANI, RYM NOURELHOUDA

$\checkmark$ MES PARENTS

$\checkmark$ MES FRĖRES ET SEEURS

$\checkmark$ TOUS MES AMIS SURTOUT ELOUARDI

$\checkmark$ ET TOUS LES HABITANTS DE LA VILLE DE HENCHIRTOUMGHANI 


\section{Résumé :}

L'utilisation des énergies renouvelables comme source de chaleur montre un double avantage : limiter la pollution et réduire le coût du combustible.

Dans les dernières décennies, la demande du froid est en forte augmentation. Surtout dans les régions dépourvues du réseau électrique.

L'une des applications est l'utilisation des machines frigorifiques à adsorption solaire. Elles utilisent le couple gel de silice-eau, c'est dans cette perspective que s'inscrit ce travail, dans l'objectif est de réaliser et expérimenter un prototype à fonctionnement cyclique.

Les résultats expérimentaux trouvés sont satisfaisante et peuvent construire une plateforme de recherche dans notre payé, dont le but est d'améliorer le rendement de l'installation expérimentale d'une part, et de maitriser ce procédé de production de froid, mal exploité en Algérie, d'autre part.

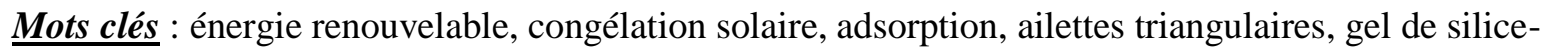
eau.

\section{$\underline{\text { Abstract : }}$}

The use of renewable energy as a heat source shows a double advantage: reduce pollution and reduce the cost of fuel.

In recent decades, the application of cold is rising sharply. Especially in regions without electricity supply.

One application is the use of solar adsorption chillers. The use of silica gel water is the task which fits the objective to realize and experience a cyclically operating prototype.

The experimental results are found satisfactory and can build a research platform in our country, which aims to improve the performance of the test facility on one hand, and to master the cold sore production process operated in Algeria.

Keywords: renewable energy, solar freezing, adsorption, triangular fins, water-silica gel

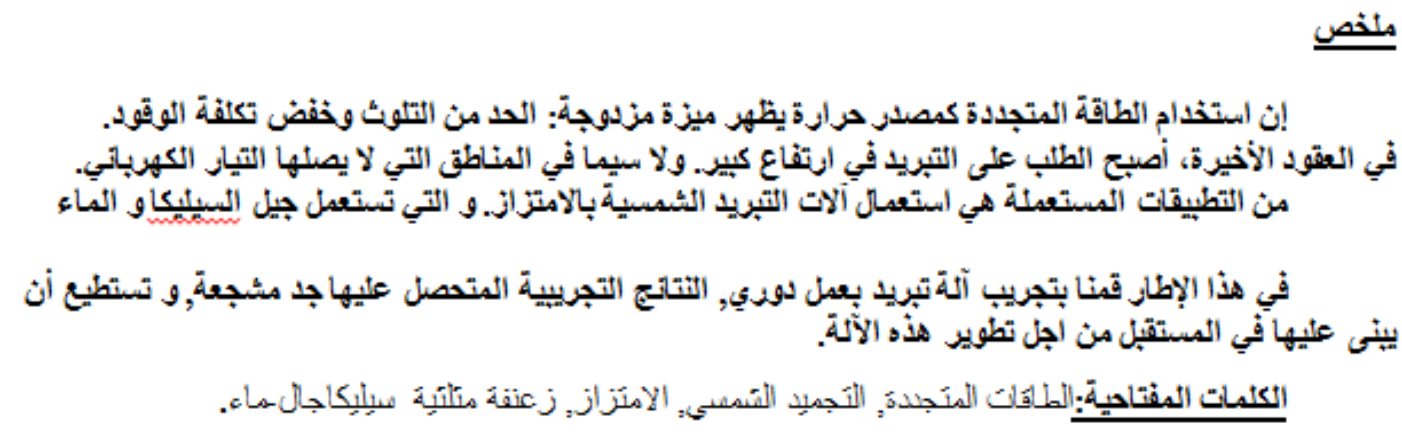




\section{Table des matières}

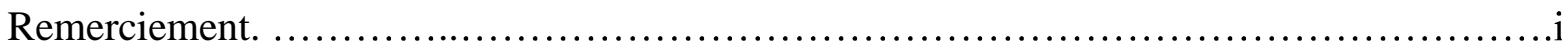

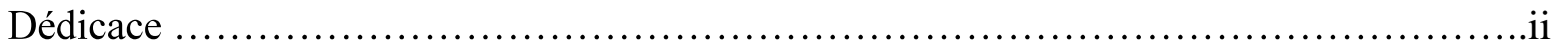

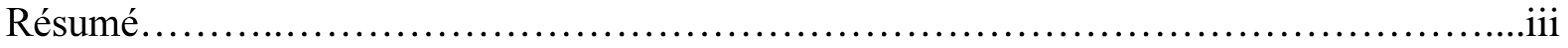

Table des matières........................................................................

Liste des figures et tableaux $\ldots \ldots \ldots \ldots \ldots \ldots \ldots \ldots \ldots \ldots \ldots \ldots \ldots \ldots \ldots \ldots \ldots \ldots \ldots \ldots, \ldots \ldots \ldots$

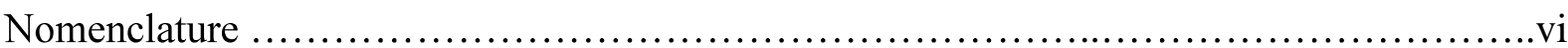

\section{Chapitre 0}

\section{Introduction générale}

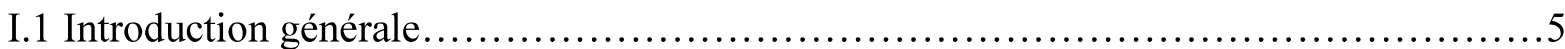

\section{Chapitre I}

\section{Etat de l'art de la production du froid solaire}

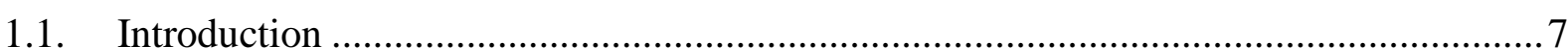

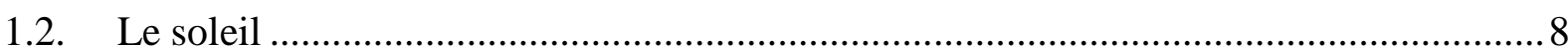

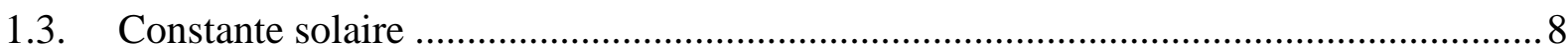

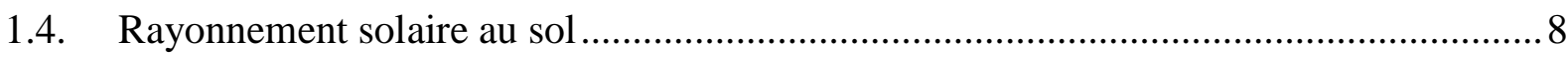

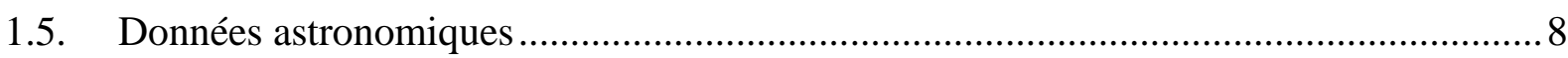

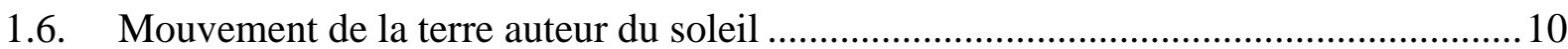

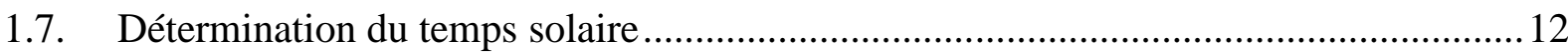


1.9. Bilan radiatif moyen

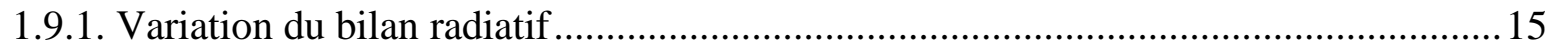

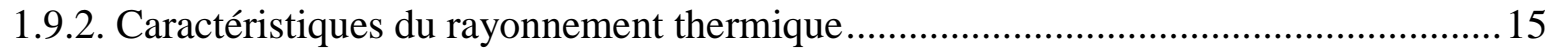

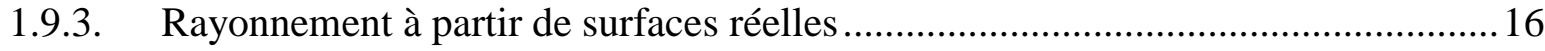

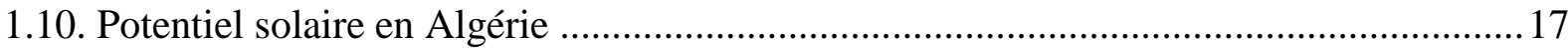

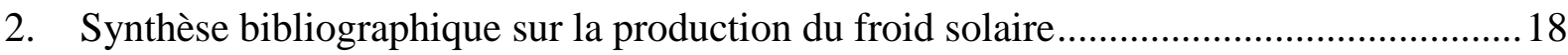

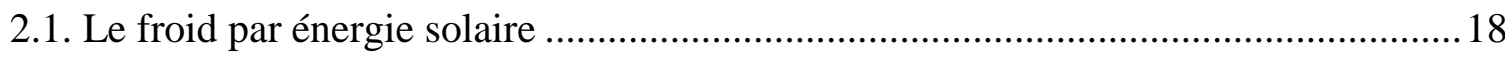

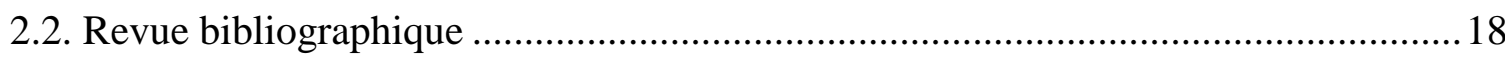

\section{Chapitre II}

\section{La machine Frigorifique solaire à Adsorption}

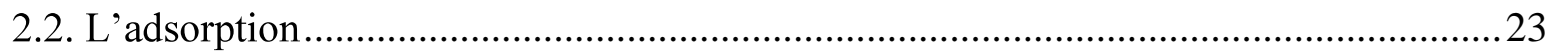

2. 3.Les Fluides frigorigènes utilisés dans le froid solaire.............................................24

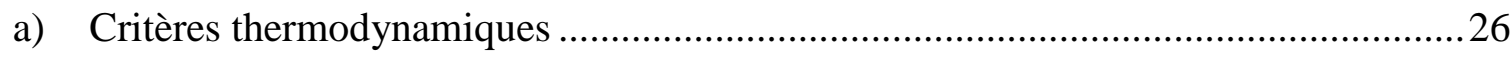

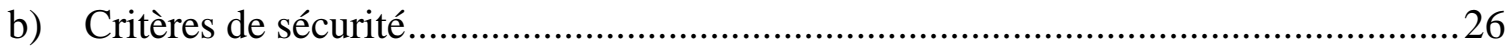

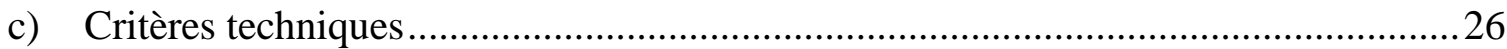

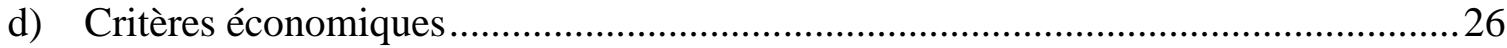

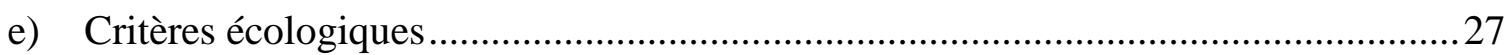

2.4. Choix du couple pour la machine frigorifique solaire à adsorption.............................27

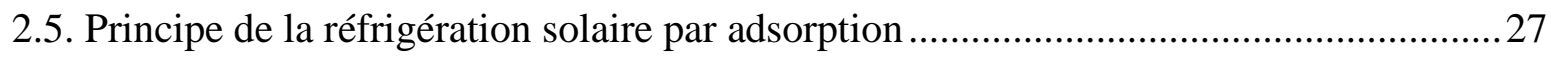

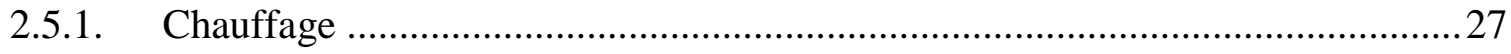

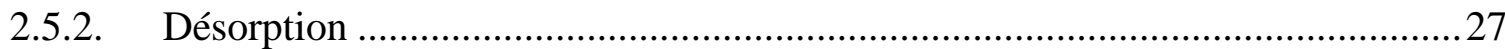

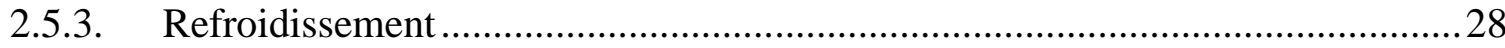

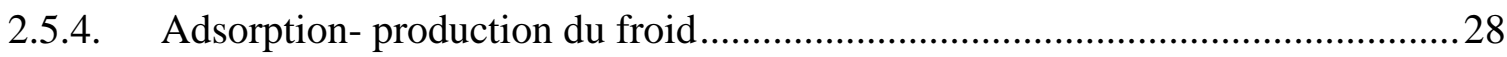

2.6. Définition des procédés thermochimiques de sorption ............................................29

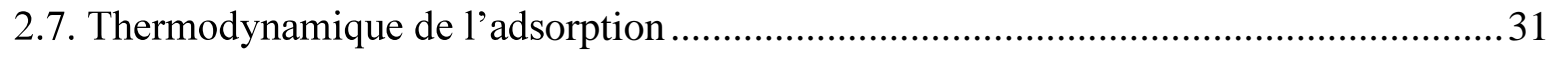

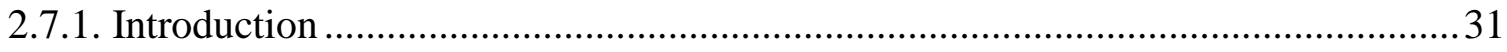

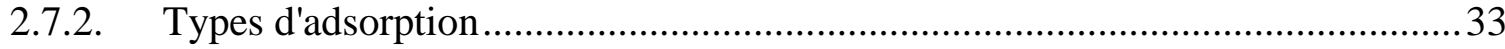

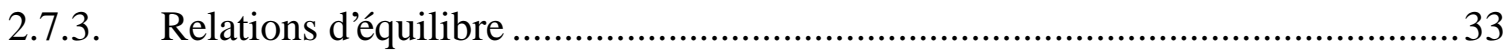

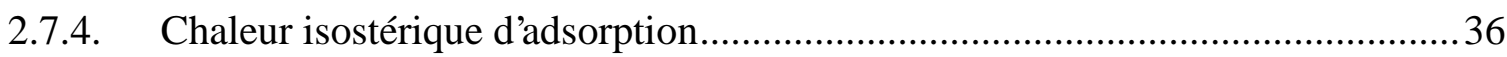




\section{Etude du capteur régénérateur de la machine frigorifique à adsorption solaire}

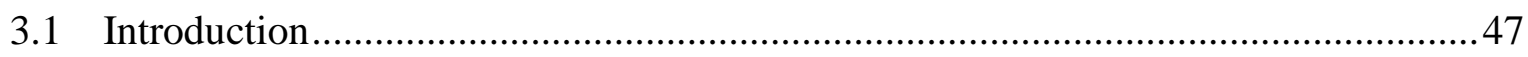

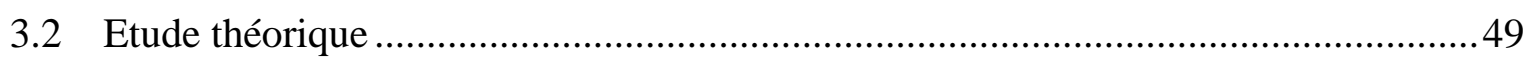

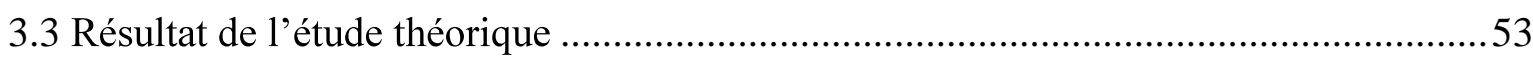

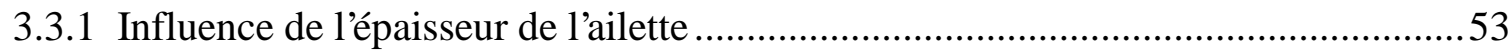

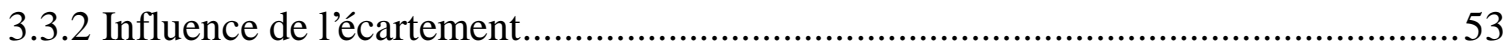

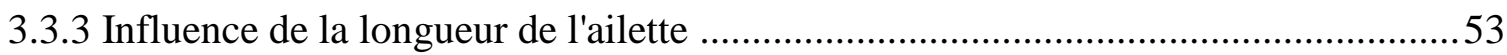

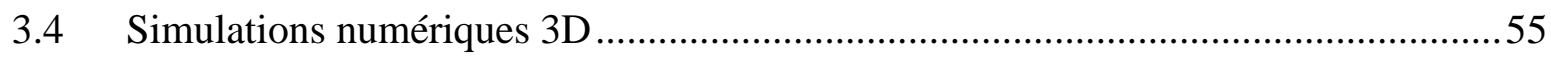

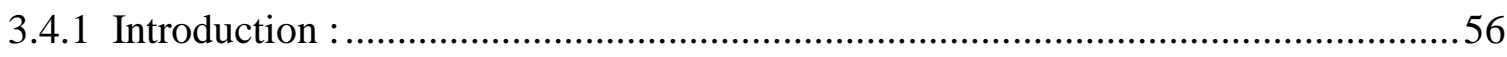

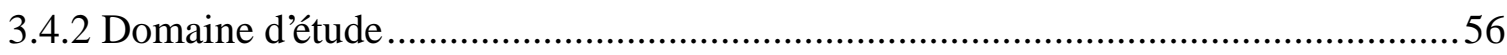

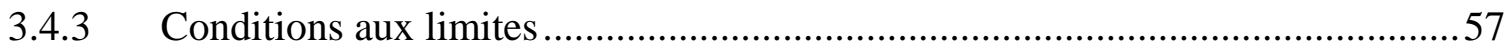

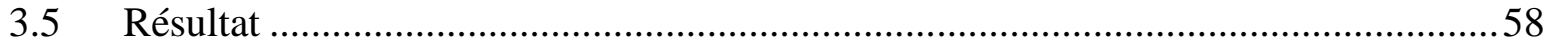

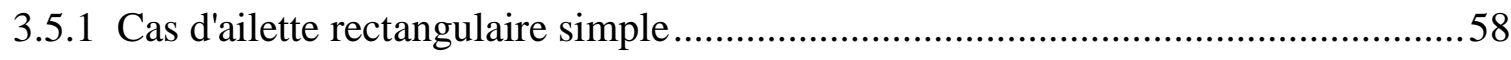

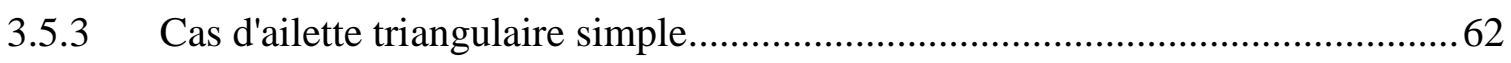

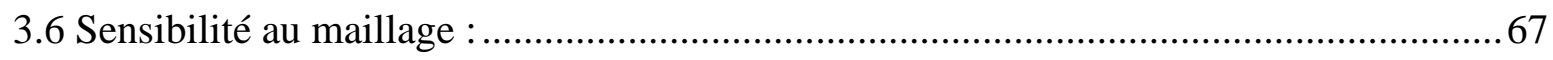

\section{Description de l'installation expérimentale et analyse des résultats}

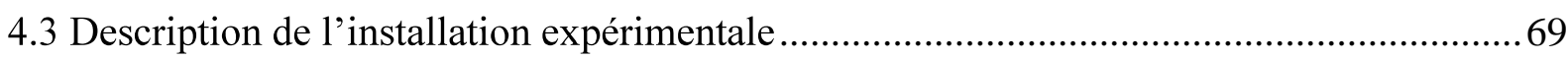

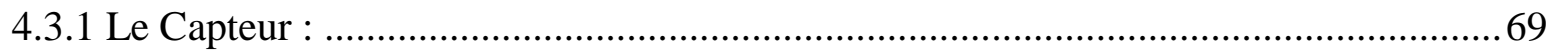

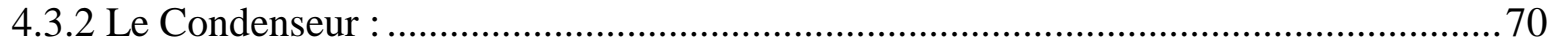

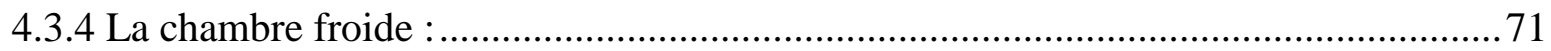

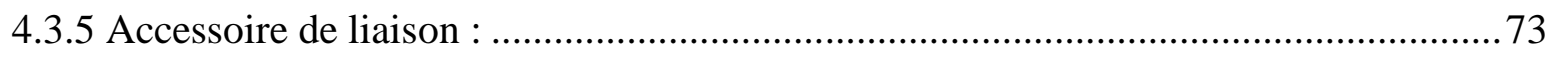

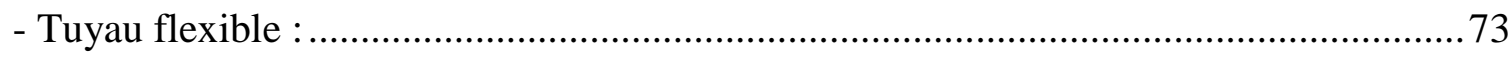

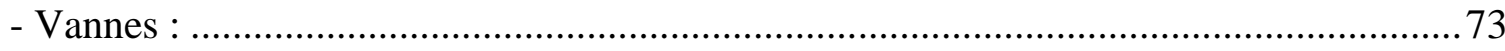

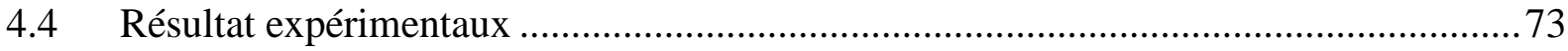

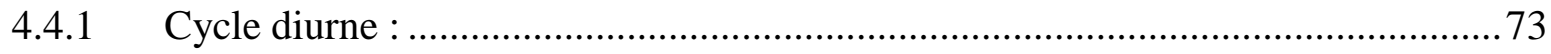

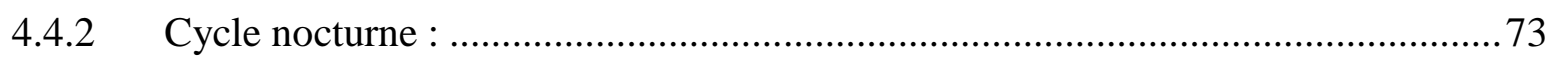

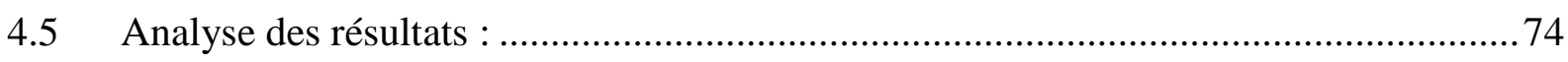


Conclusion générale

Conclusion générale et perspective.

.83

Références bibliographiques

Référence bibliographiques................................................... 85 


\section{Chapitre I}

Figure 1.1 : Coordonnées terrestres.

Figure 1.2 : Déclinaison du soleil [2]. 10

Figure1.3 : Mouvement de la terre autour du soleil [2]. 11

Figure 1.4 : Les deux systèmes de cordonnées sphérique. $\quad 12$

Figure 1.5: Distribution moyenne annuelle de l'éclairement solaire global dans le monde [5].

Figure1.7 : Flux solaire et albédo. $\quad 14$

Figure1.6 : Bilan radiatif. $\quad 15$

Figure 1.7 : Irradiation solaire globale en Algérie [8]. 17

\section{Chapitre II}

Figure 2.1 :Machine frigorifique à adsorption $\quad 22$

Figure 2.2 :Gel de silice. 23

Figure 2.3 : Schéma de principe d'une machine frigorifique solaire à adsorption :

phase de chauffage. $\quad 27$

Figure2.4 : Phase de production du froid. 28

Figure 2.5 : Fonctionnement du système de production de froid par sorption solide/gaz. 29

Figure 2.6 : Cycle théorique d'une machine à adsorption. 29

Figure2.7:Phénomène d'adsorption [39]. 31

Figure2.8 :Allure schématique des isobares d'adsorption [42]. 33

Figure 2.9:Allure schématique des isothermes d'adsorption [42]. 34

Figure. 2.10 : Schéma d'un réseau d'isostères d'adsorption [42]. 34

Figure2.11:Détermination de la chaleur isostérique d'adsorption. 36

Figure 2.12: les différents types d'isothermes d'adsorption [49]. 37

Figure2.13: Modèle d'adsorption en monocouche. 38

Figure.II.14: Représentation de Henry, Langmuir et de Freundlich de l'adsorption en monocouche 39

$\begin{array}{ll}\text { Figure.II.15: Modèle d' adsorption } & 40\end{array}$ 


\section{Chapitre III}

Figure.III.1: ailette et longueurs caractéristiques $\quad 46$

Figure.III.5: réseau d'ailette $\quad 51$

Figure.III.6 : Conditions aux limites $\quad 52$

Figure.III.7 : Profil 3D de vitesse de l'air 53

Figure.III.8 : profil de température sur le plan de symétrie $\left(\mathrm{T}\right.$ en $\left.{ }^{\circ} \mathrm{C}\right)$

Figure.III.9 b: profil de température entre deux ailettes successives vue de dessus $\quad 54$

Figure.III.9 a: profil de température entre deux ailettes successives. $1 \mathrm{~m}$ en hauteur 55

Figure.III.10: profil de température 3D 55

Figure.III.11: profil de température (dernière ailette) 56

Figure.III.12: Ailettes triangulaires

Figure.III.13: profil de vitesse de l'air plan de symétrie avant 58

Figure.III.14: profil de température plan de symétrie avant 58

Figure.III.15: profil de température entre deux ailettes successive. 59

Figure.III.16: profil de vitesse de l'air plan de symétrie. $\quad 60$

Figure.III.17: profil de vitesse de l'air entre deux ailettes successives plan ' $\mathrm{y}=1 \mathrm{~m} \perp$ $\begin{array}{ll}\text { à l'ailette'. } & 60\end{array}$

$\begin{array}{ll}\text { Figure.III.18 : sensibilité au maillage } & 61\end{array}$

\section{Chapitre IV}

Figure. IV.1 : Vue du capteur avant l'assemblage 63

Figure. IV.2 Capteur avec le boité en bois $\quad 63$

Figure. IV.3 : Condenseur à air $\quad 64$

Figure. IV.4 : panneau sandwich $\quad 65$

Figure. IV.5 : chambre froide contenant l'évaporateur 65

Figure. IV.6: photo de l'installation expérimentale 67

Figure. IV.7: Températures enregistrées journée du 01 mars 2015

Figure. IV.8 : Températures enregistrées journée du 24 mars 2015

Figure. IV.8 : Températures enregistrées journée du 27 mars 2015

Figure. IV.8 : Températures enregistrées journée du 29 mars 2015

Figure. IV.9 : Evolution du COP réel en fonction du Température ambiantes $\quad . \quad 82$ 


\section{Chapitre II}

Tableau I.1 : Potentiel solaire en Algérie.

Chapitre II

Tableau II.1 : caractéristique physique du gel de silice et le charbon actif 26

Tableau II.2 : propriété thermodynamique de quelque réfrigérants.

Chapitre IV

Tableau : IV.1 caractéristique technique du panneau sandwich 


\begin{tabular}{|c|c|c|}
\hline $\mathrm{C}$ & Condenseur & \\
\hline $\mathrm{COP}$ & Coefficient de performance & \\
\hline $\mathrm{E}$ & Epaisseur de l'ailette & {$[\mathrm{m}]$} \\
\hline$e^{*}$ & Epaisseur adimensionnel & \\
\hline $\mathrm{H}$ & Hauteur de l'ailette & {$[\mathrm{m}]$} \\
\hline $\mathrm{H}$ & Coefficient d'échange & {$\left[\mathrm{Wm}^{2} \mathrm{k}^{-1}\right]$} \\
\hline $\mathrm{h}_{0}$ & Coefficient d'échange normal & {$\left[\mathrm{Wm}^{2} \mathrm{k}^{-1}\right]$} \\
\hline $\mathrm{h}_{\mathrm{app}}$ & Coefficient d'échange apparent & {$\left[\mathrm{Wm}^{2} \mathrm{k}^{-1}\right]$} \\
\hline I & Ecartement entre deux ailettes successives & {$[\mathrm{m}]$} \\
\hline $\mathrm{L} / \mathrm{G}$ & Liquide /gaz & \\
\hline $\mathrm{La}$ & Longueur de l'ailette & {$[\mathrm{m}]$} \\
\hline $\mathrm{La}^{*}$ & Longueur de l'ailette adimensionnelle & \\
\hline $\mathrm{N}$ & Nombre d'ailette & \\
\hline $\mathrm{Nu}$ & Nombre de Nusselt & \\
\hline PCM & Matériau à changement de phase & \\
\hline $\mathrm{P}_{\mathrm{h}}$ & Haute pression & {$[\mathrm{Pa}]$} \\
\hline $\mathrm{P}_{\mathrm{b}}$ & Basse pression & {$[\mathrm{Pa}]$} \\
\hline $\mathrm{Ra}$ & Nombre de Rayleigh & \\
\hline $\mathrm{Ra}^{*}$ & Nombre de Rayleigh corrigé & \\
\hline $\mathrm{T}$ & Température & {$\left[{ }^{0} \mathrm{~K}\right]$} \\
\hline $\mathrm{X}^{*}$ & Ecartement adimensionnel & \\
\hline $\mathrm{Z}$ & Largeur de la paroi du capteur & {$[\mathrm{m}]$} \\
\hline
\end{tabular}

\section{Symbole grec}

$\begin{array}{lll}\alpha & \text { Angle d'inclinaison du capteur } & \\ \beta & \text { Coefficient de dilatation thermique } & {\left[1 /{ }^{\circ} \mathrm{K}\right]} \\ \theta & \text { Différence de température } & {\left[{ }^{\circ} \mathrm{K}\right]}\end{array}$


Efficacité de l'ailette

\section{Indice}

$\begin{array}{ll}\mathrm{a} & \text { Ambiant } \\ \mathrm{Ab} & \text { Absorption } \\ \mathrm{ad} & \text { Adsorption } \\ \mathrm{h} & \text { Haute } \\ \mathrm{b} & \text { Basse } \\ \mathrm{em} & \text { Emission }\end{array}$




\section{Introduction générale}

Le recours aux énergies renouvelables comme solution de

substitution aux énergies fossiles devient de plus en plus indispensable. Le réchauffement climatique terrestre et le tarissement progressif des ressources sont des facteurs déterminants. L'utilisation de l'énergie solaire reste limitée, surtout dans les pays du sud. Des problèmes liés à la maîtrise technologique de captation et à la transformation de cette énergie constituent un obstacle considérable à sa généralisation malgré sa disponibilité. Les avantages de ce type d'énergie ne sont plus à démontrer. Hormis, sa disponibilité et sa « gratuité », elle reste utilisable même dans les régions rurales ou sahariennes les plus isolées, et donc non raccordées au réseau électrique conventionnel.

Parmi les procédés de transformation thermique de l'énergie solaire, la réfrigération solaire. Le froid produit servira à la climatisation des locaux et au stockage des denrées alimentaires et des produits pharmaceutiques.

L'Algérie est un pays où le potentiel solaire est très important particulièrement dans les régions du sud. Avec l'accroissement de la population et le développement des infrastructures industrielles, surtout pétrolières, les besoins en froid (positif et négatif) sont en forte augmentation. 
Il reste donc à exploiter d'une façon raisonnable ce potentiel solaire afin de limiter le recours aux énergies classiques.

Plusieurs procédés de production de froid par énergie solaire sont disponibles. Les procédés thermochimiques, à absorption ou adsorption sont les plus utilisé. Leur principe est simple : Il repose sur la solubilité d'un gaz, ou d'un solide, dans un liquide qui est fonction de la pression et de la température. C'est un domaine de recherche très vaste et qui nécessite d'importants moyens expérimentaux et de calcul. Il est à signaler aussi, que le marché du solaire est très promoteur pour les années à venir. Les grandes entreprises internationales sont en concurrence pour le développement de machines avec un prix raisonnable du kW.

Plusieurs travaux dans le domaine de réfrigération solaire par adsorption ont été réalisés. C'est dans cette perspective que s'inscrit ce travail dont l'objectif est de concevoir, étudier la faisabilité et expérimenter un procédé de sorption solide/gaz permettant une production de froid inférieure à $-22^{\circ} \mathrm{C}$ en utilisant une source thermique à bas niveau de température (de l'ordre de 60 à $70^{\circ} \mathrm{C}$ ) dans le site de Biskra.

L'originalité de ce travail est de réaliser une installation plus simple que possible, en utilisant des éléments déjà disponible dans le marché national à savoir le condenseur à air fabriquer par ENIEM à Bordj Bou Arreridj, les plaques entrant dans la réalisation de la chambre froide produite par le groupe Rahmani à M'Sila

La présente thèse est constituée de quatre chapitres, d'une introduction générale qui donne une perspective générale du but envisagé et d'une conclusion générale.

Le premier chapitre est une étude bibliographique sur les notions de base sur le gisement solaire en Algérie.

Le principe de fonctionnement des machines frigorifiques solaire à adsorption sera ensuite détaillé dans le deuxième chapitre, une grande importance est donnée dans ce chapitre aux couples adsorbant-adsorbat utilisés dans le domaine de production de froid et la thermodynamique de l'adsorption.

Les problèmes rencontrés dans la phase de production de froid (cycle nocturne) nécessite une étude détaillé afin de déterminer la géométrie du capteur, élément principale de l'installation, c'est l'objet du chapitre trois.

Dans le dernier chapitre on passe à la réalisation expérimentale du prototype de la machine frigorifique solaire à adsorption et a l'analyse des résultats expérimentaux trouvés. 


\section{Chapitre 1}

\section{Etat de l'art de la production du froid solaire}

\section{Etude du gisement solaire en Algérie}

\subsection{Introduction}

Le Soleil rayonne sur la terre une puissance de $161015 \mathrm{kWh}$ par an [1], dans toutes les longueurs d'onde du spectre de la lumière visible. Une énergie renouvelable énorme et intarissable. Dans le cadre du développement durable, cette énergie renouvelable est une préoccupation d'actualité surtout si elle est exploitée sur le lieu de conversion. Les thèmes de développement durable et de la maîtrise de l'énergie sont chaque jour plus vitaux. Les besoins énergétiques et leurs couvertures à long terme jouent dans ce contexte un rôle central. En effet, les grandes surfaces et l'éloignement des agglomérations du sud rendent le coût des installations d'énergie électrique très élevé. Cependant, les applications énergétiques sont nombreuses et variées, et les exigences technologiques sont plus en plus grandes.

La puissance moyenne reçue du soleil par la terre est d'environ $20 \%$ du flux lumineux compte tenu du Jour et de la nuit, de la latitude de l'endroit, des saisons et de la couverture nuageuse. L'utilisation des énergies renouvelables en Algérie, n'a pas dépassé le seuil d'expérience durant trois décennies d'indépendance, malgré la situation géographique, l'Algérie dispose d'un des gisements solaire les plus élevés au monde. La durée d'insolation sur la quasi-totalité du territoire national dépasse les 2000 heures annuellement et peut atteindre les 3900 heures (hauts plateaux et Sahara). L'énergie reçue quotidiennement sur une surface horizontale de 
$1 \mathrm{~m}^{2}$ est de l'ordre de $5 \mathrm{KWh}$ sur la majeure partie du territoire national, soit près de $1700 \mathrm{KWh} / \mathrm{m}^{2} / \mathrm{an}$ au Nord et $2263 \mathrm{kWh} / \mathrm{m}^{2} / \mathrm{an}$ au Sud [1].

\subsection{Le soleil}

Le soleil est une sphère gazeuse composée presque totalement d'hydrogène, son diamètre est de $1391000 \mathrm{~km}$, qui est 100 fois plus grand que celui de la terre, sa masse est par contre de l'ordre de $2.10^{27}$ tonnes [2].

Toute l'énergie du soleil provient des réactions thermonucléaires qui s'y produisent, elles transforment à chaque seconde 564.106 tonnes d'hélium, la différence de 4 millions de tonnes est dissipée sous forme d'énergie $\left(E=m . c^{2}\right)$, ce qui représente une énergie totale de $36.10^{22}$ KW. La terre étant à une distance de $150.10^{6} \mathrm{Km}$ du soleil, elle reçoit une énergie de $1,8 \cdot 10^{17} \mathrm{~W}$ [2]. On notera que $98 \%$ du rayonnement solaire est émis dans des longueurs d'onde inférieures à $4 \mu \mathrm{m}$, et comme première approximation, le rayonnement solaire peut être assimilé au rayonnement d'un corps noir à une température de 5777 K [2].

\subsection{Constante solaire}

L'énergie totale que le soleil envoie à la limite de l'atmosphère terrestre sur une surface de $1 \mathrm{~m}^{2}$ placée perpendiculairement au rayonnement est appelée ',constante solaire '..Elles est fondamentale et indépendante des conditions météorologiques [2].

\subsection{Rayonnement solaire au sol}

On est intéressé, du point de vue utilisation de l'énergie, par l'énergie reçue au voisinage du sol qu'à la limite de l'atmosphère terrestre. Le rayonnement solaire arrivant au sol se compose de:

$\checkmark$ Rayonnement direct : C'est celui qui traverse l'atmosphère sans subir de modifications.

$\checkmark$ Rayonnement diffus : C'est une partie du rayonnement solaire diffusé par les particules solides ou liquides en suspension dans l'atmosphère, il n'a pas de direction privilégiée.

$\checkmark$ Rayonnement global : C'est la somme du rayonnement direct et diffus.

\subsection{Données astronomiques}

Dans ce qui suite nous allons présenter les définitions de quelques paramètres astronomiques.

a) L'équateur: C'est une ligne fictive de séparation entre l'hémisphère nord et l'hémisphère sud (Figure 1.1). 
b) Latitude du lieu $(\Phi)$ : Présente la distance angulaire d'un point quelconque du globe par rapport l'équateur (de $0^{\circ}$ à $90^{\circ}$ dans l'hémisphère nord), mesuré en degré.

c) La longitude $(\varphi)$ : C'est l'angle formé par le méridien de Greenwich et le méridien du lieu considéré. La longitude est comprise entre -180 (vers l'ouest) et +180 (vers l'est). Comme la terre met 24 heures pour faire un tour sur elle-même $\left(360^{\circ}\right)$, chaque heure représente $15^{\circ}$ d'écart de longitude et donc, chaque degré de longitude représente 4 minutes [3].

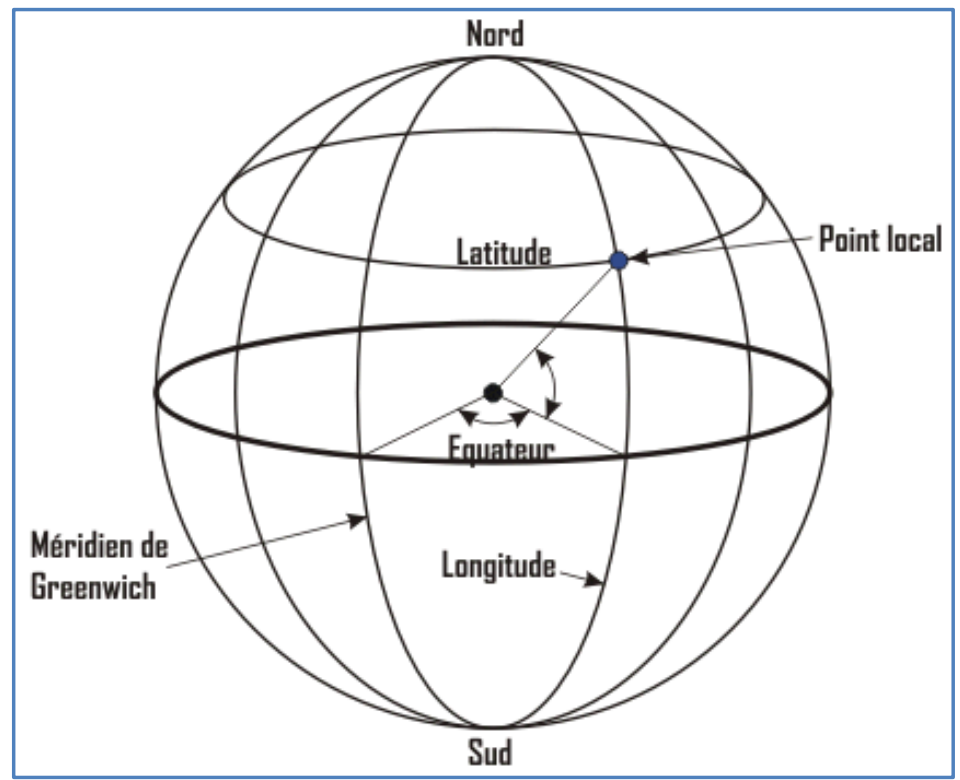

Figure 1.1 : Coordonnées terrestres.

d) L'altitude (z) : C'est la distance verticale exprimé en mètres, séparant le point considéré du relief terrestre du niveau de la mer, pris comme surface de référence.

e) La déclinaison $(\delta)$ : C'est l'Angle entre la direction terre soleil et le plan équatorial de la terre ou bien la latitude du lieu où le soleil est à la verticale à midi solaire. Cet angle varie de $-23^{0} 27^{\prime}$ au solstice d'hiver à $23^{0} 27^{\prime}$ au solstice d'été et il est nul aux équinoxes. La déclinaison qui est fonction du jour de l'année est définie par son quantième $(\mathrm{dj})$, pour le premier janvier $\mathrm{dj}=1$ et ainsi de suite, Elle est donnée par l'expression suivante :

$$
\sin \delta=0.398 \sin [0.986(\mathrm{dj}-82)] \text { ou bien } \delta=23.45 \sin [0.986(284+\mathrm{dj})]
$$


Les angles sont exprimés en degré.

f) L'albédo : C'est la fraction de l'énergie incidente diffusée par un corps lumineux, pour un corps noir parfait.

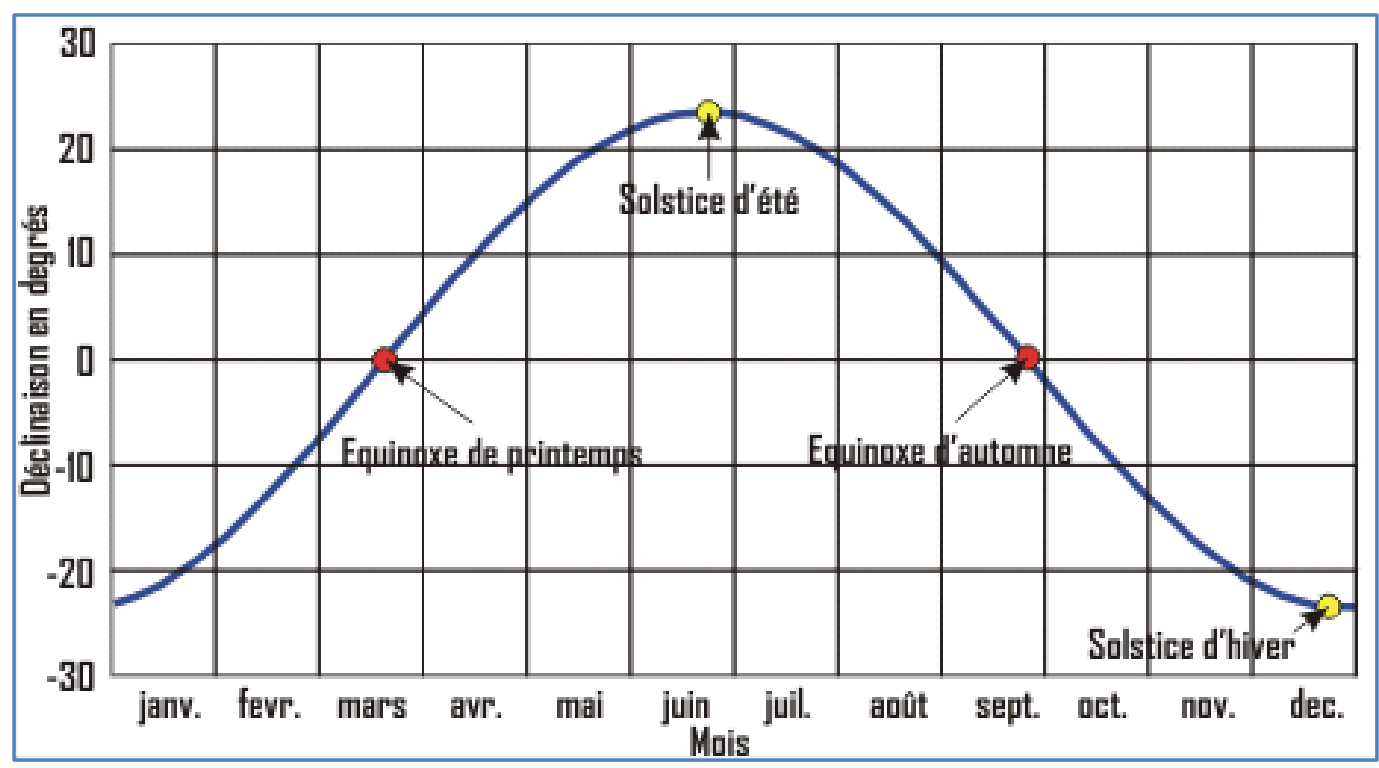

Figure 1.2 : Déclinaison du soleil [2].

\subsection{Mouvement de la terre auteur du soleil}

La trajectoire de la terre autour du soleil est une ellipse dont le soleil est l'un des foyers. Le plan de cette ellipse appelé l'écliptique (Figure 1.3).

L'excentricité de cette ellipse est faible. Ce qui fait que la distance terre- soleil ne varie que de $\pm 1.7 \%$ par rapport à la distance moyenne qui est de $149675.10^{6} \mathrm{~km}$.

La terre tourne également sur elle-même autour d'un axe appelé l'axe des pôles. Le plan perpendiculaire à l'axe des pôles et passant par le centre de la terre est appelé l'équateur. L'axe des pôles n'est pas perpendiculaire à l'écliptique : l'équateur et l'écliptique font entre eux un angle appelé inclinaison et qui vaut $23^{\circ} 27^{\prime}$ [2]. 


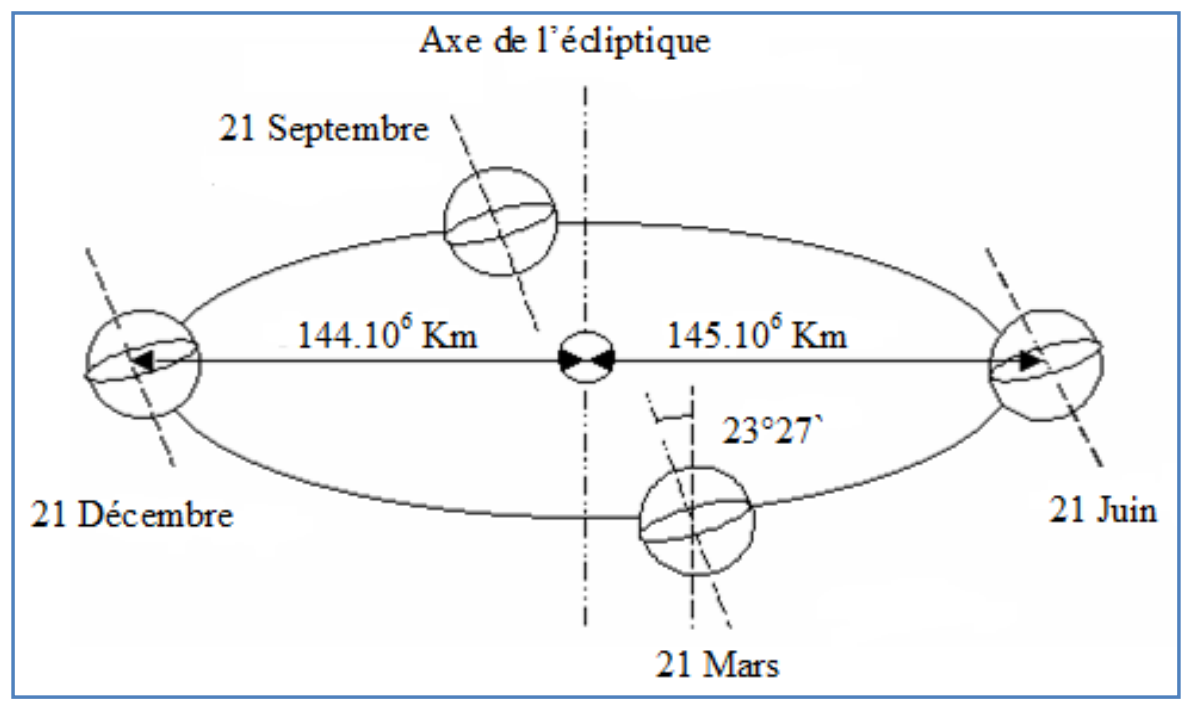

Figure1.3 : Mouvement de la terre autour du soleil [2].

Le repérage du soleil s'effectue par l'intermédiaire de deux angles :

a) Hauteur angulaire du soleil (h) : La hauteur du soleil est l'angle entre la droite joignant le centre du disque solaire ou point d'observations et le plan horizontal, passant par le point d'observations.

$$
\sin h=\sin \Phi \cdot \sin \delta+\cos \Phi \cdot \cos w \cdot \operatorname{Cos} \delta
$$

b) L'azimut $(\omega)$ :C'est L'angle mesuré dans le sens des aiguilles d'une montre entre le point cardinal sud (dans l'hémisphère sud) et la projection sur le plan horizontal local de la droite reliant la terre au soleil. L'angle est mesuré dans le sens des aiguilles d'une montre clans l'hémisphère nord et dans le sens contraire dans l'hémisphère sud. En utilisant les projections sur le plan horizontal du point d'observation [3].

$$
\sin \omega=\cos \delta \cdot \sin \omega / \cos \mathrm{h}
$$

Où $\omega$ est l'angle horaire solaire.

Ces deux angles sont représentés sur la Figure (1.4). 


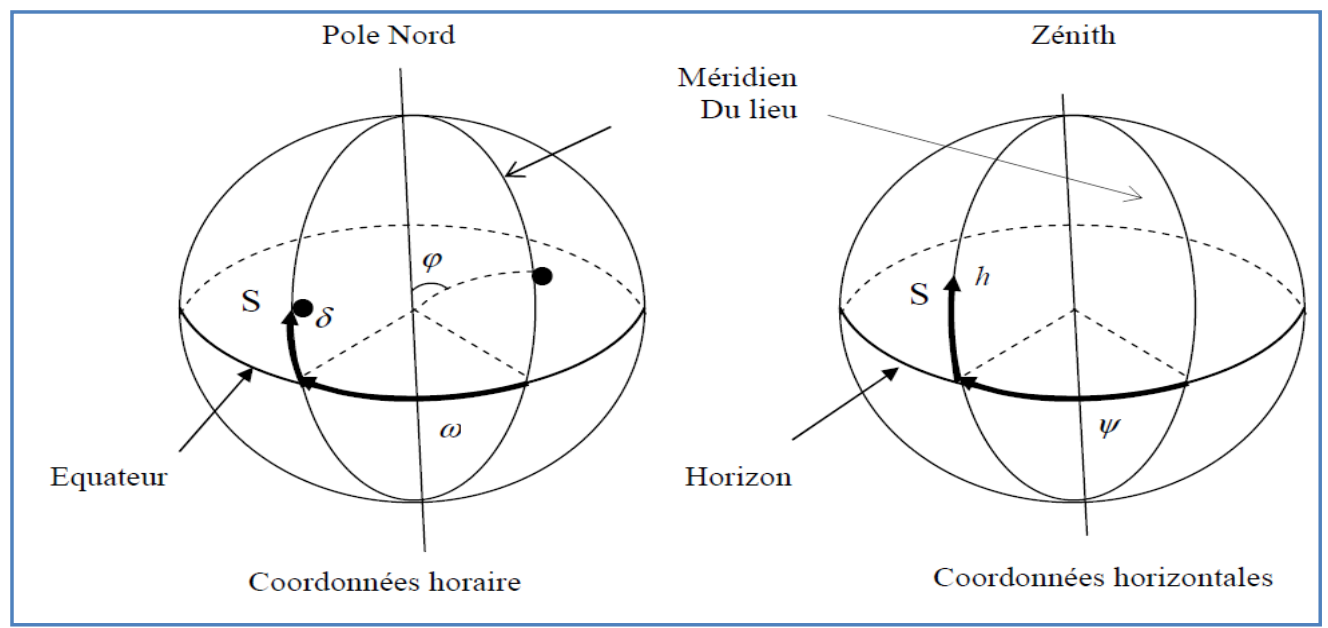

Figure 1.4 : Les deux systèmes de cordonnées sphérique.

\subsection{Détermination du temps solaire}

Toutes les valeurs du temps dans le calcul d'énergie solaire sont exprimées en temps solaire vrai [3].

a) Le temps solaire vrai (TSV) : C'est l'angle dièdre du méridien du soleil et du lieu à un instant donné (TSV) qui est donné par les cadrans solaires et le temps.

$$
\mathrm{TSV}=12-\omega / 15 \text { (heures) }
$$

Où $\omega$ est l'angle horaire formé par le plan méridien passant par le centre du soleil et le plan vertical du lieu méridien, il définit le temps solaire :

Il est midi TSV, si $\omega=0$.

b) Le temps solaire moyen(TSM) : Il est appelé parfois temps local, qui est en relation avec TSV.

$$
\mathrm{TSV}-\mathrm{TSM}=\mathrm{ET}
$$

Où, ET est l'équation de temps (min).Elle est donnée par la relation :

$$
\begin{gathered}
\mathrm{ET}=9,87 \sin (2 . n)-7.53 \cos \mathrm{n}-1,5 \sin \mathrm{n} \\
\mathrm{n}=\left(\mathrm{n}_{\mathrm{j}}-81\right) .360 / 365
\end{gathered}
$$

$\mathrm{n}_{\mathrm{j}}$ est le numéro du jour dans l'année.

c) Le temps civil : c'est le temps qui à comme origine minuit. 
d) Le temps universel (TU) : est le temps civil moyen du méridien de Greenwich (méridien origine) appelé encore Greenwich Mean Time (GMT), pour un lieu situé à la longitude $(\varphi)$.

$$
\begin{aligned}
& \text { TU=TSM- }(\varphi / 15) \text { pour les longitudes Est } \\
& \text { TU=TSM- }(\varphi / 15) \text { pour les longitudes Ouest }
\end{aligned}
$$

e) Le temps légal ou local : C'est une heure liée au "TU" par une différence fixe exprimé en un nombre entier d'heures qui dépend de la longitude, et des coutumes de chaque pays.

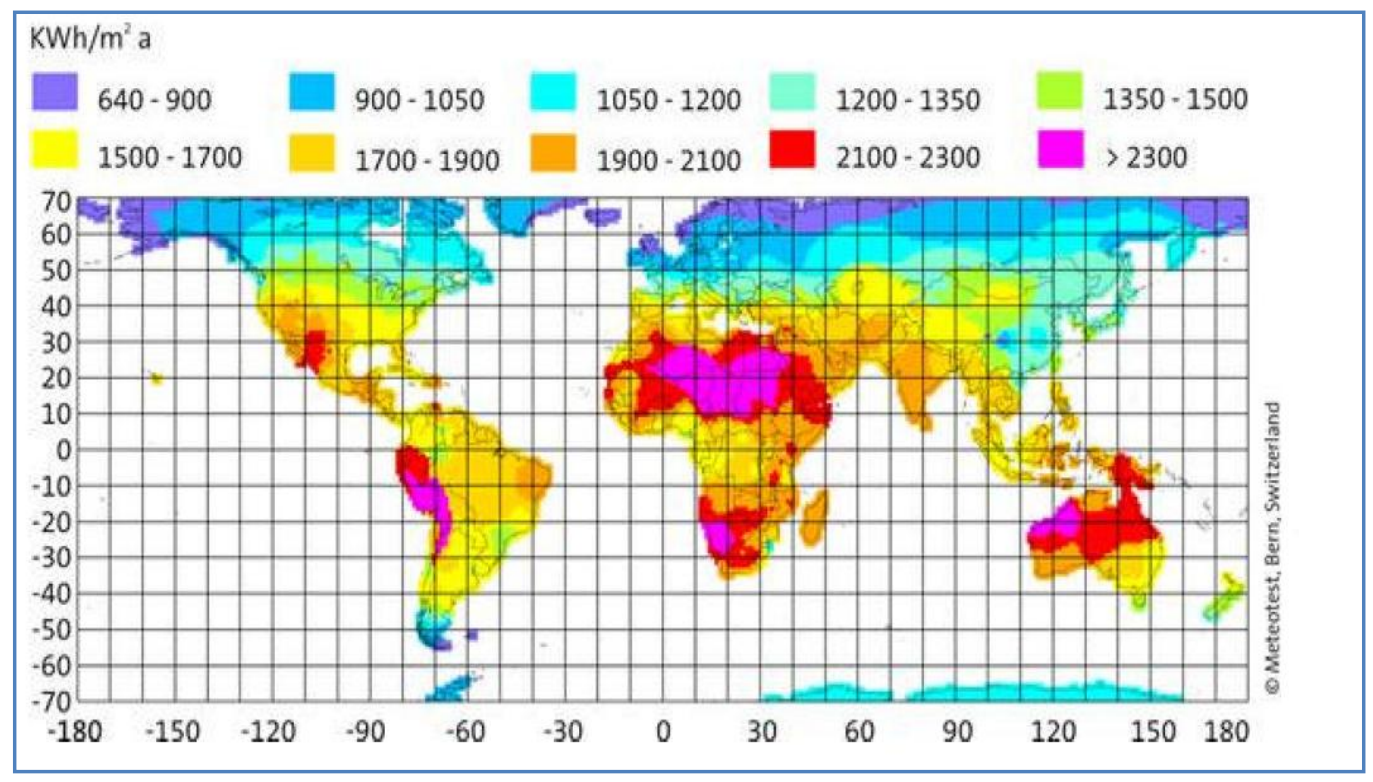

Figure 1.5: Distribution moyenne annuelle de l'éclairement solaire global dans le monde [5].

\subsection{Flux solaire et albédo}

La surface du soleil a une température d'environ $6000{ }^{\circ} \mathrm{K}$. Elle émet des radiations dont la longueur d'onde varie de 0,2 à $4 \mu \mathrm{m}$, c'est-à-dire depuis l'ultra-violet jusqu'au proche infrarouge. Le domaine visible s'étend de 0,3 à $0,7 \mu \mathrm{m}$ environ.

En moyenne : $30 \%$ du flux est réfléchi ou diffusé vers l'espace (par les nuages, les aérosols et la surface de la Terre) ; 70 \% est absorbé par l'atmosphère et par la surface et transformé en chaleur. Cette portion d'énergie solaire convertie en chaleur est le moteur des mouvements des masses d'air et d'eau à la surface du globe, et donc des climats. La Terre, dont la température 
superficielle est beaucoup plus basse, émet à son tour un flux d'infrarouges de forte longueur d'onde (de 3 à $30 \mu$ m environ). [6]

L'état thermique du système Terre-Atmosphère est relativement stationnaire sur une période de plusieurs années : le bilan radiatif global est nul.

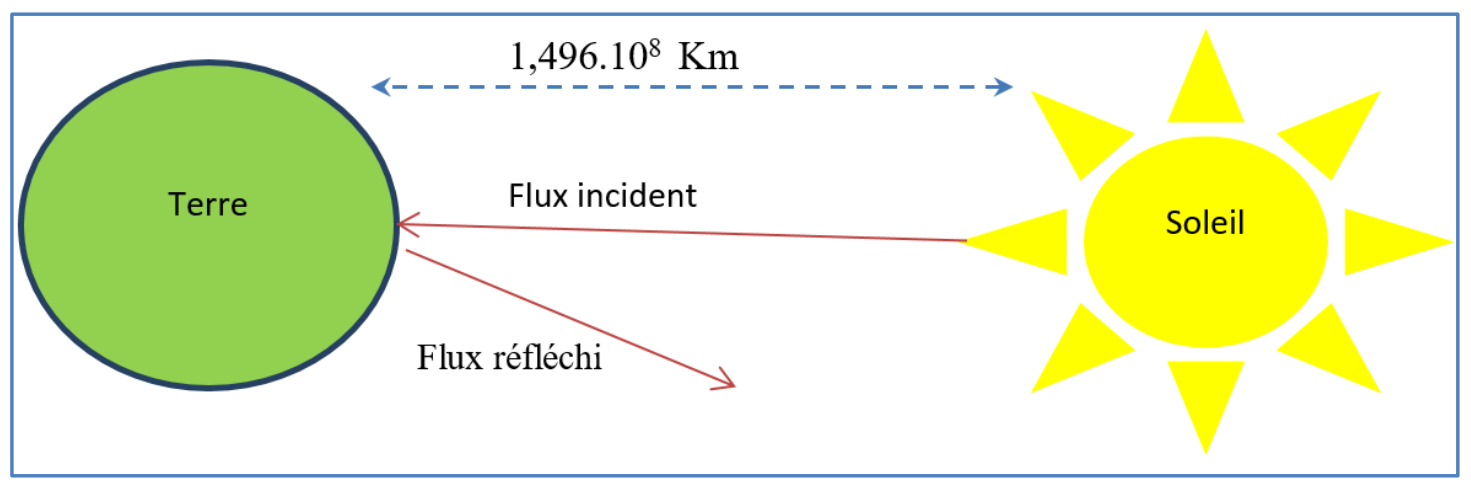

Figure1.7 : Flux solaire et albédo.

Remarque : Albédo =flux réfléchi/ flux incident

L'albédo est en moyenne $30 \%$ pour le globe :

$\checkmark 5-10 \%$ sur les mers sans nuages ;

$\checkmark 10-15 \%$ au-dessus des forêts ;

$\checkmark 30-50 \%$ sur les déserts ;

$\checkmark 60-85 \%$ sur la neige et la glace.

\subsection{Bilan radiatif moyen}

Avec un albédo de 30\%, 70\% du rayonnement solaire est absorbé et transformé en chaleur : Les Ultra-violets sont absorbés par l'ozone de la stratosphère Les Infrarouges sont absorbés par la vapeur d'eau et le gaz carbonique de la troposphère puis réémis vers le sol et vers l'espace. Le Visible et le proche Infrarouge atteignent la surface [6]. 
Sans absorption des radiations par l'atmosphère, la température moyenne globale serait de -18 ${ }^{\circ} \mathrm{C}$ au lieu de $15{ }^{\circ} \mathrm{C}$ : c'est l'effet de serre. Les principaux gaz à effet de serre sont : H2O, CO2, $\mathrm{N}_{2} \mathrm{O}, \mathrm{CH}_{4}, \mathrm{O}_{3}$, Fréons [7]. Le bilan radiatif est nul à l'échelle du globe et pour un an. En revanche il change selon le lieu et la saison. Le flux solaire incident varie en fonction de la latitude, de la saison et de l'heure. L'albédo varie en fonction de la latitude et de la saison.

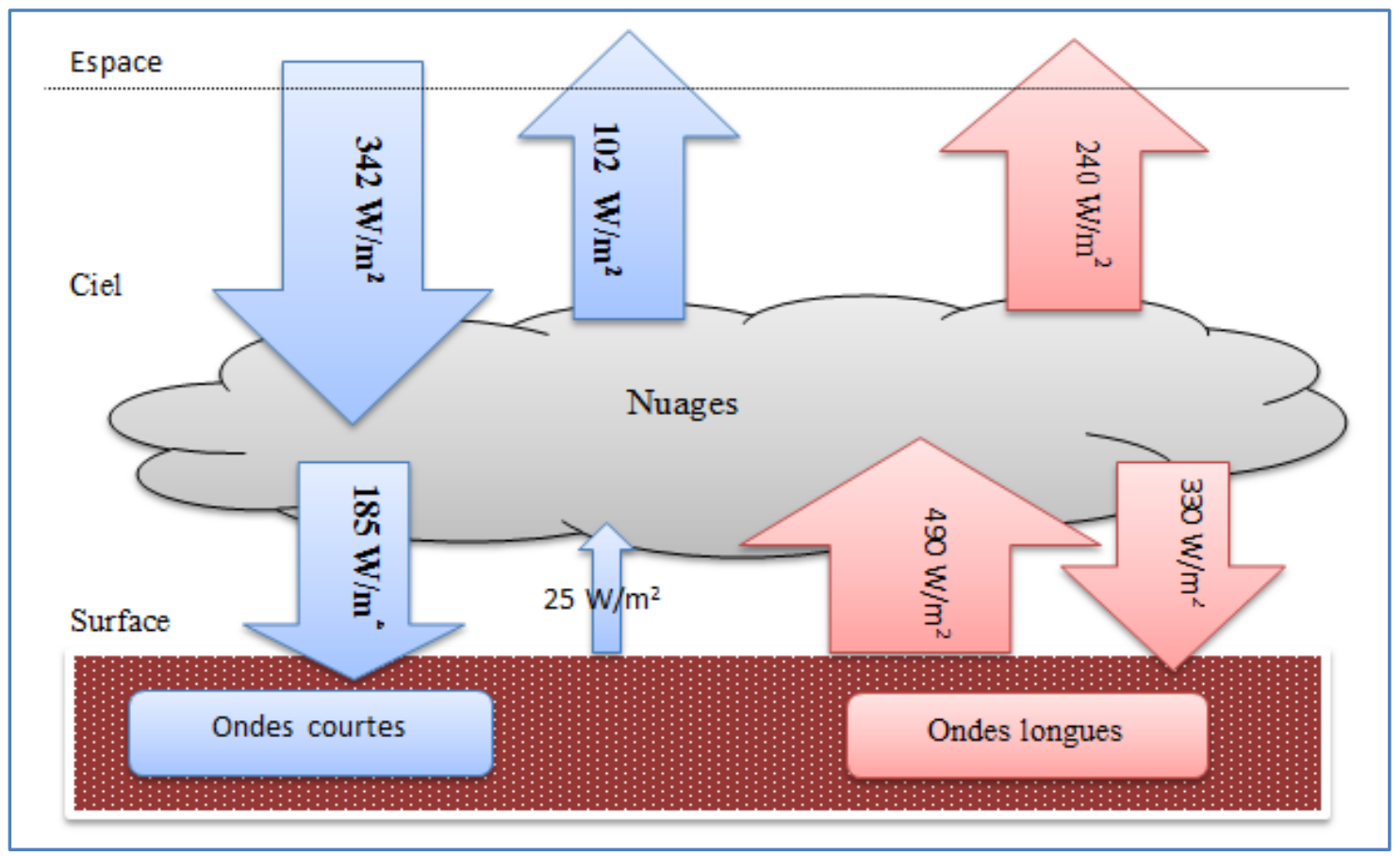

Figure1.6 : Bilan radiatif.

\subsubsection{Variation du bilan radiatif}

\section{- Variations saisonnières}

Le cycle annuel du bilan radiatif local est plus ou moins marqué selon la latitude.

$\checkmark$ Les saisons sont déterminées par l'orientation de l'axe de rotation de la Terre par rapport au plan de l'écliptique et la position de la Terre par rapport au soleil.

Actuellement, l'axe de rotation est incliné de $23^{\circ}$ environ, en direction de l'étoile polaire.

Remarque : si l'axe de rotation était perpendiculaire au plan de l'écliptique, le soleil serait toujours au zénith au-dessus de l'équateur et le bilan radiatif serait constant localement tout le long de l'année avec une orbite circulaire.

L'énergie solaire est répartie de façon très inégale à la surface du globe. Elle est redistribuée par convection selon des transferts méridiens; la chaleur excédentaire de l'équateur est 
distribuée aux latitudes élevées par la circulation atmosphérique et par les courants océaniques.

\subsubsection{Caractéristiques du rayonnement thermique}

Émission, absorption, réflexion-diffusion, transparence et opacité.

Nous allons étudier les différents comportements de la matière vis à vis du rayonnement thermique. Nous rappelons que le rayonnement thermique est un rayonnement électromagnétique, on retrouve donc le vocabulaire des ondes.

$\checkmark$ Émission: un corps porté à une certaine température convertit son énergie interne (énergie microscopique) en rayonnement thermique, une unité de surface d'un corps émet durant une unité de temps une quantité d'énergie appelée flux d'émission. On le note $\mathrm{F}_{\mathrm{emi}}$.

$\checkmark$ Absorption : Il s'agit de l'opération inverse. Quand une surface reçoit un flux d'énergie, la fraction transformée en énergie interne est appelée flux absorbé (noté $\mathrm{F}_{\mathrm{abs}}$ ).

$\checkmark$ Réflexion et diffusion : Au lieu d'être absorbé, le rayonnement incident sur une paroi peut être directement renvoyé par la paroi.

Dans ces conditions on distingue 2 cas :

1. Le renvoi obéit aux lois de l'optique géométrique (un angle d'incidence, un angle de réflexion). Il s'agit alors de réflexion.

2. Le renvoi se fait dans toutes les directions (même si l'on a une seule direction incidente). On parle alors de diffusion.

$\checkmark$ Transparence et opacité : Un milieu peut transmettre intégralement l'onde incidente, il est alors appelé milieu transparent. Le vide est un exemple de milieu transparent. En première approximation, le verre est aussi un milieu transparent pour des longueurs d'ondes dans le domaine du visible. Inversement, un corps ne transmettant aucune partie du rayonnement incident n'est dit corps opaque.

$\checkmark$ Flux incident : Le flux incident $F_{i}$ est défini comme la puissance surfacique du rayonnement incident en un point considéré de la surface du corps étudié Le flux incident est soit réfléchi-diffusé, soit absorbé. On a donc la relation suivante. :

$$
\mathrm{F}_{\mathrm{i}}=\mathrm{F}_{\text {ref }}+\mathrm{F}_{\mathrm{abs}}
$$

Flux partant : Le flux surfacique partant du corps est lui la somme du flux émis et du flux réfléchi. On a donc la relation suivante : 


$$
\mathrm{F}_{\mathrm{p}}=\mathrm{F}_{\mathrm{ref}}+\mathrm{F}_{\mathrm{emi}}
$$

\subsubsection{Rayonnement à partir de surfaces réelles}

Une surface réelle diffère du Corps Noir pour :

L'absorption : Une surface réelle est caractérisée par son pouvoir absorbant. On note $\alpha$ le coefficient d'absorption. Il s'agit du rapport entre le flux absorbé et le flux incident :

$$
\alpha=\mathrm{F}_{\mathrm{abs}} / \mathrm{F}_{\mathrm{i}}
$$

Ce rapport dépend de la longueur d'onde du rayonnement incident.

Pour le Corps Noir, il est égal à 1 .

L'émission : Une surface réelle est caractérisée par son pouvoir d'émission. On note e le coefficient d'émission.

Il s'agit du rapport entre le flux émis réel et le flux émis du corps noir de même température.

$$
e=F_{\text {ref }}^{\text {réel }} / F_{\text {emi }}^{C N}=F_{\text {ref }}^{\text {réel }} / \sigma T^{4}
$$

Ce rapport dépend de la longueur d'onde du rayonnement émis. Pour le Corps Noir, il est égal à 1.Pour une même longueur d'onde, le coefficient d'absorption et le coefficient d'émission sont égaux. Cependant, le rayonnement incident et le rayonnement émis étant souvent dans des domaines de longueur d'ondes très différents, il faudra prendre 2 valeurs différentes [6,7].

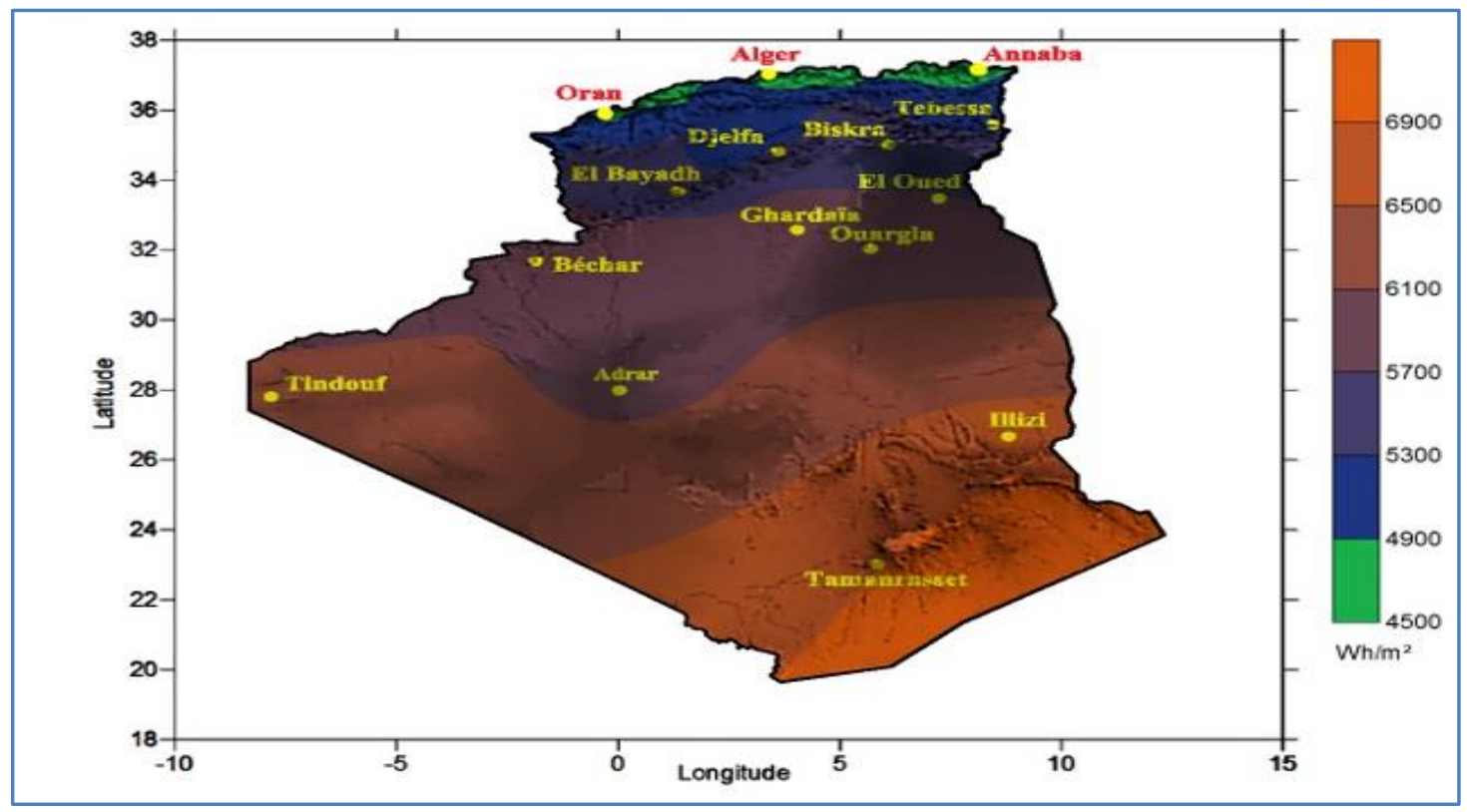

Figure 1.7 : Irradiation solaire globale en Algérie [8]. 


\subsection{Potentiel solaire en Algérie}

L'Algérie possède un gisement solaire parmi les plus élevé dans le monde, la durée moyenne d'ensoleillement dans le Sahara algérien est de 3500 heures, ce potentiel peut constituer un facteur important de développement durable dans cette région, s'il est exploité de manière économique, le tableau suivant indique le taux d'ensoleillement pour chaque région de l'Algérie.

\begin{tabular}{|l|c|c|c|}
\hline Région & Régions Côtières & Haut plateaux & Sahara \\
\hline Superficie & $4 \%$ & $10 \%$ & $86 \%$ \\
\hline Durée moyenne d'ensoleillement (Heures/an) & 2650 & 3000 & 3500 \\
\hline Énergie moyenne reçue (KWh/m2) & 1700 & 1900 & 2650 \\
\hline
\end{tabular}

Tableau 1.1 : Potentiel solaire en Algérie [8].

Le volet de l'énergie solaire le plus utilisé dans notre pays est le solaire photovoltaïque, les autres volets solaire thermique et thermodynamique restent toujours au stade d'expériences.

\section{Synthèse bibliographique sur la production du froid solaire}

\subsection{Le froid par énergie solaire}

Dans le domaine du rafraîchissement solaire (production de froid positif) plusieurs techniques ont pris la place des systèmes à adsorption classique et à compression. Ce sont les systèmes à adsorption $\mathrm{H} 2 \mathrm{O} / \mathrm{LiBr}$ [9], (production d'eau glacée $7^{\circ} \mathrm{C}$ ) les systèmes à adsorption Silicagel/eau, les cycles de refroidissement d'air par déshumidification.

Dans la réfrigération solaire entre 0 et $-10^{\circ} \mathrm{C}$ quelques systèmes seulement sont capables de produire du froid négatif, ils utilisent l'ammoniac, qui est totalement neutre pour l'environnement, comme fluide de travail. Ce sont des systèmes à sorption liquide/gaz : NH3/H2O [10], ou solide/gaz : SrCl2/NH3 [11,12], CaCl2NH3 [13]. Ces systèmes permettent une production de froid à $-10^{\circ} \mathrm{C}$ (glace solaire), leur inconvénient majeur réside dans la nécessite d'une température de source chaude minimale de $120^{\circ} \mathrm{C}$, or ce niveau de température demande une technologie très avancées ce qui rend le coût total de l'installation trop élevé. 
Dans le domaine de la congélation solaire $\left(\mathrm{t}^{\circ}=-20^{\circ} \mathrm{C}\right)$, à notre connaissance, aucun système mis à part les systèmes à compression, n'a été testé, du fait des températures très élevées requises par la source chaude.

Les études préliminaires ont été faites récemment dans des Laboratoires internationaux dans le cadre de la définition d'un nouveau concept de procédé basé sur la sorption solide/gaz par réaction chimique. Ce procédé permet de produire du froid à $-22^{\circ} \mathrm{C}$ (avec un évaporateur à$30^{\circ} \mathrm{C}$ ), tout en utilisant de la chaleur à basse température de l'ordre de $65 / 70^{\circ} \mathrm{C}$ [14].

L'intérêt d'utiliser des capteurs solaires basses températures est d'obtenir des rendements de captation élevés de l'ordre de $50 \%$ à 70\%, Ainsi le coefficient de performance solaire (SCOP) de l'installation de congélation solaire, rapport de l'énergie frigorifique produite à l'énergie solaire captée pourrait atteindre une valeur de10 à 20\%, supérieure à celle des systèmes actuellement développés oscillants entre 8 et $20 \%$.

L'objectif n'est pas seulement de développer un procédé innovant de congélation solaire, mais aussi de proposer un système qui répond aux exigences socio-économique; un coût global faible, une simplicité technologique (systèmes sans vanne, auto adaptif aux conditions extérieures).

\subsection{Revue bibliographique}

Pour les applications de réfrigération (production de froid à des températures inférieures à $\left(5^{\circ} \mathrm{C}\right)$, les procédés de sorption nécessitent des températures de source chaude supérieures à $90^{\circ} \mathrm{C}$.

Tchernev a développé dès les années 1970 un réfrigérateur solaire basé sur l'adsorption zéolithe-eau (Anyanwu, 2003) [15]. Le COP du système réalisé était de 0,15. Les températures de génération du sorbant et de froid produit étaient respectivement de l'ordre de $120^{\circ} \mathrm{C}$ et $3^{\circ} \mathrm{C}$ pour une pression inférieure à 0,004 bar durant la phase de nuit. Ce premier prototype a été suivi de nombreux autres, notamment ceux de Guilleminot et Meunier (1979 à 1986),Boubakri, Grenier et Pons (1985 à 1987), ou Critoph (1986 à 1993) [16] (Dieng et Wang 2001) [17].

Enibe et Iloeje (1997) [18].ont développé un dispositif de production de glace utilisant un capteur solaire plan double vitrage. Ce prototype à sorption chimique fonctionne avec le 
couple $\mathrm{CaCl} 2-\mathrm{NH} 3$. Les températures atteintes lors des phases de désorption sont de l'ordre de 80 à $100^{\circ} \mathrm{C}$ pour un test réalisé au Niger. Ce système a permis de produire de la glace avec un COP solaire expérimental allant jusqu'à 0,06 .

Un autre prototype solaire à réaction chimique solide-gaz entre SrCL2 et NH3 a été conçu et testé en Inde (New Delhi) par Bansal et alen 1997.Ce prototype a permis d'atteindre des températures de production de froid de $-4^{\circ} \mathrm{C}$ après 3 jours de refroidissement de la chambre froide, pour des températures de régénération du sorbant comprises entre 100 et $130^{\circ} \mathrm{C}$. Le COP solaire du cycle a atteint une valeur de 0.08 au cours des journées étudiées [19].

Les procédés solaires à sorption ont fait l'objet d'importantes études au cours des dernières années en Chine. Ce pays présente en effet des régions étendues qui ne bénéficient pas d'approvisionnement électrique sûr. Les machines solaires constituent une véritable alternative d'avenir pour la conservation des denrées dans ces régions. De nombreux prototypes ont été testés.

Des systèmes de production de glace ou de production hybride de froid et l'eau chaude sanitaire par adsorption ont été développés, à partir des couples charbon actif-méthanol ou charbon actif-éthanol et de sources chaudes atteignant des températures de l'ordre de $100^{\circ} \mathrm{C}$. Les COP expérimentaux des prototypes varient entre 0.06 et 0.15 (Li et al, 2002b, Wang 2001 2002, Li et al, 2004a et 2004b, Li et al, 2005) [20,21, 22].

Récemment, Anyanwu et Ezekwe (2003) ont développé au Niger un prototype de production de glace par le procédé d'adsorption charbon actif-méthanol. Un capteur à simple vitrage a été utilisé. Ils n'ont réussi qu'à atteindre une température de $1{ }^{\circ} \mathrm{C}$ pour des températures de régénération du sorbant comprises entre 80 et $100^{\circ} \mathrm{C}$. Le COP du cycle est de 0.056 à 0.093 et le COP solaire de 0.007 à 0.015 au cours des journées étudiées [23].

Un autre prototype à adsorption fonctionnant avec la même paire de réactifs et le même type de capteur solaire également été testé au Burkina-faso (Butcher et al, 2003) [24]. Il a permis de produire de la glace et le COP solaires expérimentaux obtenus sur les 20 jours de tests varient de 0.087 à 0.131 .

En suisse, un prototype haut performance avec le couple Silicagel-Eau utilisant un capteur solaire plan simple vitrage a été développé. Le sorbant est placé directement au sein du 
capteur solaire qui présente également la caractéristique de pouvoir s'ouvrir au cours de la nuit (par ouverture de la face arrière et de l'isolant du capteur) afin d'augmenter la convection naturelle et de favoriser le refroidissement du sorbant au cours de la nuit. Ce système de production de glace et de stockage de froid a présenté des performances intéressantes, avec un COP solaire de 0.16 (Hilbdrand et al, 2004) [25].

Ainsi, les procédés solaires à sorption font actuellement l'objet d'un intérêt de certaines entreprises. Ces procédés, qui permettent d'obtenir des températures de production de froid supérieurs à $-5^{\circ} \mathrm{C}$, sont actuellement développés à l'échelle préindustrielle pour des applications de rafraîchissement, de climatisation ou encore de production de glace.

Pour la production du froid négatif, il n'existe actuellement aucun procédé à sorption de production de froid à très basse température à partir de capteurs solaires basse température. En effet, les procédés qui ont été expérimentés peuvent produire du froid à des températures inférieures à $-10^{\circ} \mathrm{C}$ mais nécessitent des sources chaudes à des températures élevée. Cependant, on peut noter quelques réalisations présentées récemment qui utilisent des ressources basse température.

Tamainot et Critoph (1997) ont développé un réfrigérateur à adsorption utilisant le couple charbon actif-ammoniac. Leur système a permis de produire du froid à des températures jusqu'à $-19^{\circ} \mathrm{C}$, mais la température de source chaude nécessaire était encore élevée dans ce cas [26].

Erhard et Hahne (1997 et Erhard et al, 1998) ont développé un procédé solaire à sorption utilisant la réaction chimique entre l'ammoniac et le chlorure de strontium $\mathrm{SrCl} 2$. Le COP du système variait entre 0.045 et 0.082 pour des températures de froid jusqu'à $-10^{\circ} \mathrm{C}$, mais en utilisant des capteurs solaires à concentration [27,28].

Berlitz et al (1998) ont développé un réfrigérateur fonctionnant avec le couple ammoniac-eau. Les températures de froid produit ont atteint $-20^{\circ} \mathrm{C}$, mais le système nécessite une température de source chaude très élevée [29].

Best et Pilatowsky (1998) évoquent des expériences menées en Californie produisant du froid à des températures situées entre -20 et $-10^{\circ} \mathrm{C}$, grâce aux technologies d'absorption eauammoniac et ammoniac-nitrate de lithium, mais en utilisant de la vapeur géothermale [30]. 
Ces mêmes auteurs évoquent le développement au Mexique d'une technologie d'adsorption eau-ammoniac pour la congélation rapide et la conservation du poisson. Ce système a permis d'obtenir des températures de l'ordre de $-40^{\circ} \mathrm{C}$, mais nécessite une source chaude à 150$160^{\circ} \mathrm{C}$ à partir de capteurs solaires paraboliques Wang (2001), enfin, fait référence à un système de production de froid par adsorption charbon actif-méthanol permettant d'obtenir des températures d'évaporation de l'ordre de -13.5 à $-15,5^{\circ} \mathrm{C}$, utilisant de la chaleur à des températures variant de 90 à $100^{\circ} \mathrm{C}[31]$. 


\section{Chapitre 2}

\section{Fonctionnement d'une machine Frigorifique à adsorption}

\subsection{La machine Frigorifique solaire à Adsorption}

Les machines frigorifiques solaire à adsorption constituent un sujet de recherche intéressant et actuel. Elles constituent une alternative valable aux machines à compression. L'intermittence du cycle de base d'une machine à adsorption représente une caractéristique utile dans les applications où l'énergie n'est que discontinuement disponible. Ces machines sont donc parfaitement adaptables à l'énergie solaire.

Dans une machine frigorifique à adsorption, l'énergie solaire peut être directement absorbée par le réacteur-adsorbeur (contenant l'adsorbant solide) situé à l'intérieur du capteur solaire. 
Cependant la présence du solide adsorbant qui est un mauvais conducteur de chaleur, et la nature de contact entre l'adsorbant et les parties métalliques du réacteur, font que l'énergie captée par la surface absorbante du collecteur se diffuse mal à l'intérieur du réacteur. Ceci entraînera de forts gradients de température et une différence importante de température entre la paroi métallique et le milieu réactif. Il en résulte une augmentation des pertes avant du capteur (qui sont directement liées à la température de la paroi métallique), et par conséquent une baisse des performances de la machine [32].

L'amélioration des performances de ce type de machine est conditionnée donc par la qualité de transfert thermique dans le réacteur. Pour atteindre cet objectif, deux voies peuvent être adoptées :

$\checkmark$ Amélioration des propriétés de transfert de chaleur de l'adsorbant.

$\checkmark$ Amélioration de la surface d'échange entre la paroi métallique extérieure du réacteur et l'adsorbant solide.

\subsection{L'adsorption}

L'adsorption est le phénomène général résultant de l'interaction modérée entre un solide, l'adsorbant, et un gaz. S'il s'agit, comme ici, d'adsorption physique, le gazgarde son identité, même s'il perd quelques degrés de liberté [33].

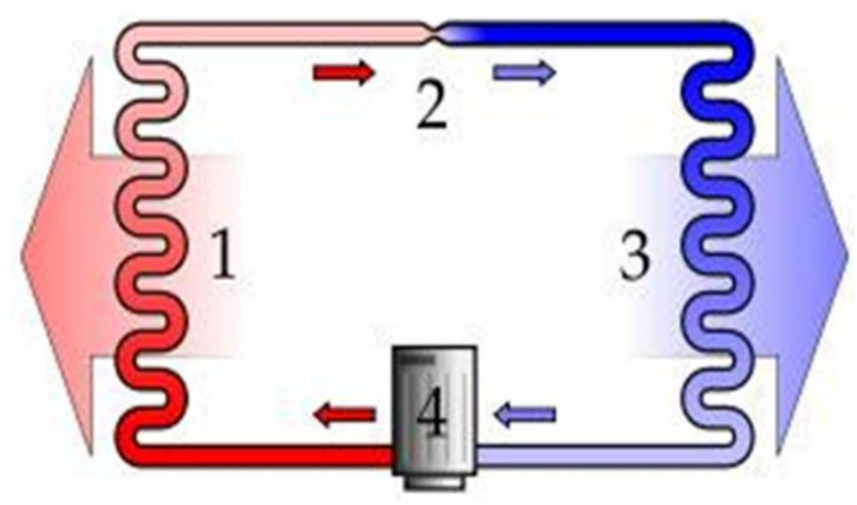

Figure 2.1 :Machine frigorifique à adsorption

1- Condenseur ; 2- Détendeur ; 3-Evaporateur ; 4-Compresseur.

Pour les applications considérées, quelques propriétés sont importantes, l'interaction est plus énergétique que la chaleur latente liquide-gaz; cette interaction est très spécifique sur le plan moléculaire (eau, ammoniac, azote, etc...). 
$\checkmark$ Le phénomène est réversible et rapide;

$\checkmark$ Le comportement reste bivariant, favorisé par la pression et défavorisé par la température.

On peut considérer tous solide comme adsorbant, alors que seules ceux qu'ils ont une surface spécifique supérieure à $100 \mathrm{~m}^{2} / \mathrm{g}$, peuvent être utilisés dans la production du froid. On peut utiliser comme adsorbant :

Charbon actif : Fabriquer généralement à partir du bois, du charbon, de la noix de coco, etc..., par un traitement thermique allant jusqu'à $1000^{\circ} \mathrm{C}$ pour créer la microporosité [34]. Cet adsorbant est disponible dans le marché algérien mais malheureusement avec un prix très élevé (14000DA/kg).

$\checkmark$ Gel de silice : Ou silicagel est un hydroxyde de silicium $\mathrm{Si}(\mathrm{OH})_{4}$ polymère d'acide silicique préparer à partir de silicate de sodium. On a opté pour cet adsorbant vu la quantité d'eau qu'il peut adsorber (à une pression de vapeur d'eau proche de la saturation $(400 \mathrm{~g} / \mathrm{kg})$ [34], sa disponibilité dans le marché national avec des prix très intéressant $(14000 \mathrm{DA} / \mathrm{kg})$ présente un autre avantage.

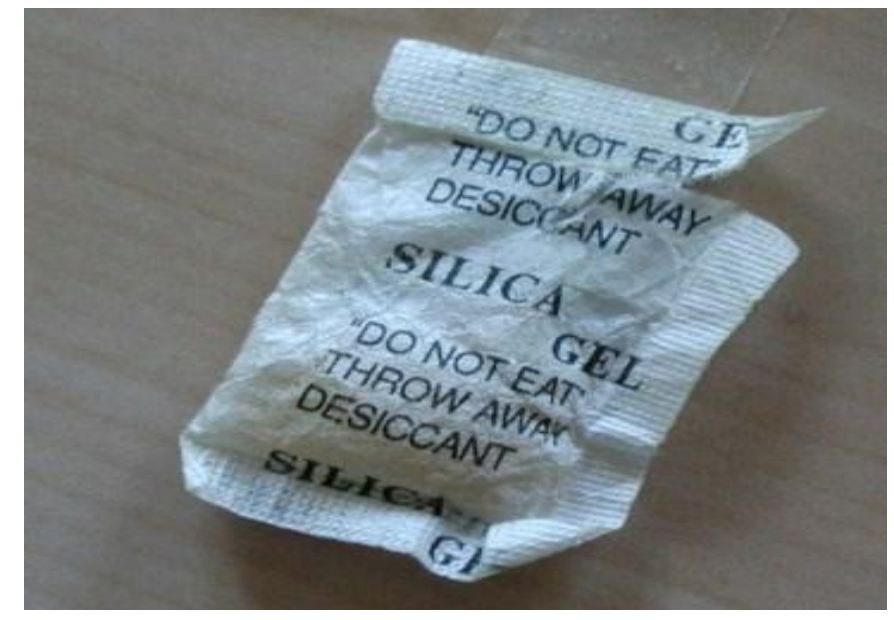

Figure 2.2 :Gel de silice.

\section{3.Les Fluides frigorigènes utilisés dans le froid solaire}

Les frigorigènes sont des substances qui évoluent dans les systèmes frigorifiques dont leur rôle est d'assurer le transfert de chaleur entre la source froide et la source chaude. 
Pour les systèmes de production de froid par adsorption ou réaction chimique, un nombre important de couples peut être choisi (Wongsuwan et al (2001) [36]. De même, dans le domaine de l'adsorption, une étude a montré que le nombre de fluide possible s'élevait à 40 environ et les adsorbants à 200. Les couples peuvent être classés en fonction du gaz choisi.

\begin{tabular}{|c|c|c|r|}
\hline Adsorbant & Surface spécifique $\left(\mathbf{m}^{2} / \mathbf{g}\right)$ & Taille des pores $(\mathbf{n m})$ & Porosité interne \\
\hline Gel de silice & 600 à 800 & 2,0 à 5,0 & 0,4 à 0,5 \\
\hline Charbon actif & 400 à 2000 & 1,0 à 4,0 & 0,4 à 0,8 \\
\hline
\end{tabular}

Tableau 2.1 : Caractéristique physique du gel de silice et le charbon actif [35].

Le méthanol $(\mathrm{CH} 3 \mathrm{OH})$ est un gaz envisageable qui est adsorbé par le charbon actif. Il a été de nombreuses fois étudié et utilisé au cours des dernières années. Cependant, la décomposition du méthanol à des températures supérieures à $150^{\circ} \mathrm{C}$ impose une prise de précautions pour son utilisation dans des applications solaires.

L'eau présente l'avantage d'être stable sur une large gamme de température et de pression, d'avoir une grande enthalpie de vaporisation, d'être abondante, neutre et non toxique. Cependant, les pressions opératoires sont très faibles. Donc sa mise en œuvre nécessite des technologies de vide, de plus, sa température de solidification ne permet pas de l'utiliser pour des applications très basses températures.

L'ammoniac a été couramment utilisé à la fois pour des procédés de chauffage comme pour la production du froid. Ce fluide est stable sur une large gamme de température et de pression. Il présente une enthalpie de vaporisation élevée, et son point de congélation est de $-77^{\circ} \mathrm{C}$, à la pression atmosphérique. De plus, il est relativement abondant et de coût limité. Cependant, à haute température, la pression de l'ammoniac est élevée, et ce fluide est volatil, toxique et corrosif. En ad- et absorption, les couples eau-ammoniac et charbon actif-ammoniac sont utilisés fréquemment pour des applications basses température. Dans les procédés à sorption chimique, l'ammoniac a été largement étudié et expérimenté, surtout avec des sels de type chlorure [37]. 


\begin{tabular}{|c|c|c|c|}
\hline \multirow{3}{*}{ Réfrigérant } & Température $\left({ }^{\circ} \mathbf{C}\right)$ & $\begin{array}{c}\text { Chaleur latentede } \\
\text { vaporisation }\left(\mathbf{k J ~ k g}^{-1}\right)\end{array}$ & Pression vapeur (bar) \\
\cline { 2 - 4 } & 0 & 2485 & 0.0063 \\
\cline { 2 - 4 } & 20 & 2442 & 0.0236 \\
\hline \multirow{3}{*}{ Ammoniac } & 40 & 2397 & 0.0747 \\
\cline { 2 - 4 } & -30 & 1359 & 1.195 \\
\cline { 2 - 4 } & -10 & 1296 & 2.909 \\
\hline \multirow{3}{*}{ Méthanol } & 30 & 1262 & 4.294 \\
\cline { 2 - 4 } & -50 & 1146 & 11.665 \\
\cline { 2 - 4 } & -10 & 1190 & 0.01 \\
\hline
\end{tabular}

Tableau 2.2 : Propriété thermodynamique de quelque réfrigérants [38].

Le choix d'un frigorigène dépend de plusieurs critères, qui sont :

\section{a) Critères thermodynamiques}

$\checkmark$ Température d'ébullition.

$\checkmark$ Température critique.

$\checkmark$ Échauffement des vapeurs lors de la compression

b) Critères de sécurité

$\checkmark$ Inflammabilité de la vapeur des frigorigènes dans l'air

$\checkmark$ Toxicité

$\checkmark$ Action sur les denrées alimentaires

c) Critères techniques

$\checkmark$ Action sur les métaux

$\checkmark$ Action sur les matières plastiques

$\checkmark$ Comportement en présence d'eau

Stabilité thermique

$\checkmark$ Aptitude aux fuites et leurs détections 
d) Critères économiques

$\checkmark$ Incidence sur la prise de l'installation

$\checkmark$ Coût du frigorigène

$\checkmark$ Disponibilité

e) Critères écologiques

$\checkmark$ Action sur la couche d'ozone

$\checkmark$ Influence sur la couche d'ozone et effet de serre.

\subsection{Choix du couple pour la machine frigorifique solaire à adsorption}

Le couple réfrigérant-adsorbant à une grande importance sur l'efficacité des machine frigorifique solaire à adsorption, à ce stade l'adsorbant doit être choisi selon, sa conductivité thermique : pour avoir un bon transfert de chaleur dans l'adsorbant.

$\checkmark$ Sa surface d'adsorption : elle doit être grande ;

$\checkmark$ Sa disponibilité : c.à.d. disponible dans le marché avec des prix raisonnable.

\subsection{Principe de la réfrigération solaire par adsorption}

Une machine frigorifique solaire à adsorption fonctionne en circuit fermé. Elle est constituée d'un capteur plan contenant l'adsorbant et jouant un double rôle (captation et dégagement de la chaleur). Il est relié à un condenseur et à un évaporateur. Le principe de fonctionnement de ces machines repose sur les phénomènes de sorption - désorption d'un gaz dans un solide. Cette réaction chimique est exo ou endothermique suivant son sens de déroulement.

Le fonctionnement de la machine peut être divisé en quatre phases : chauffage, désorption, refroidissement et l'adsorption-production du froid.

\subsubsection{Chauffage (du $7 \mathrm{~h}$ au $10 \mathrm{~h})$}

Le rayonnement solaire chauffe le capteur- adsorbeur qui va à son tour chauffer l'adsorbant. La pression de vapeur et la température de système augmentent progressivement. C'est la phase transitoire de montée en température

\subsubsection{Désorption(du $10 \mathrm{~h}$ au $17 \mathrm{~h}$ )}

La pression de saturation correspondant à la température du condenseur est maintenant atteinte. Les vapeurs se condensent dans le condenseur et s'écoulent vers l'évaporateur. 


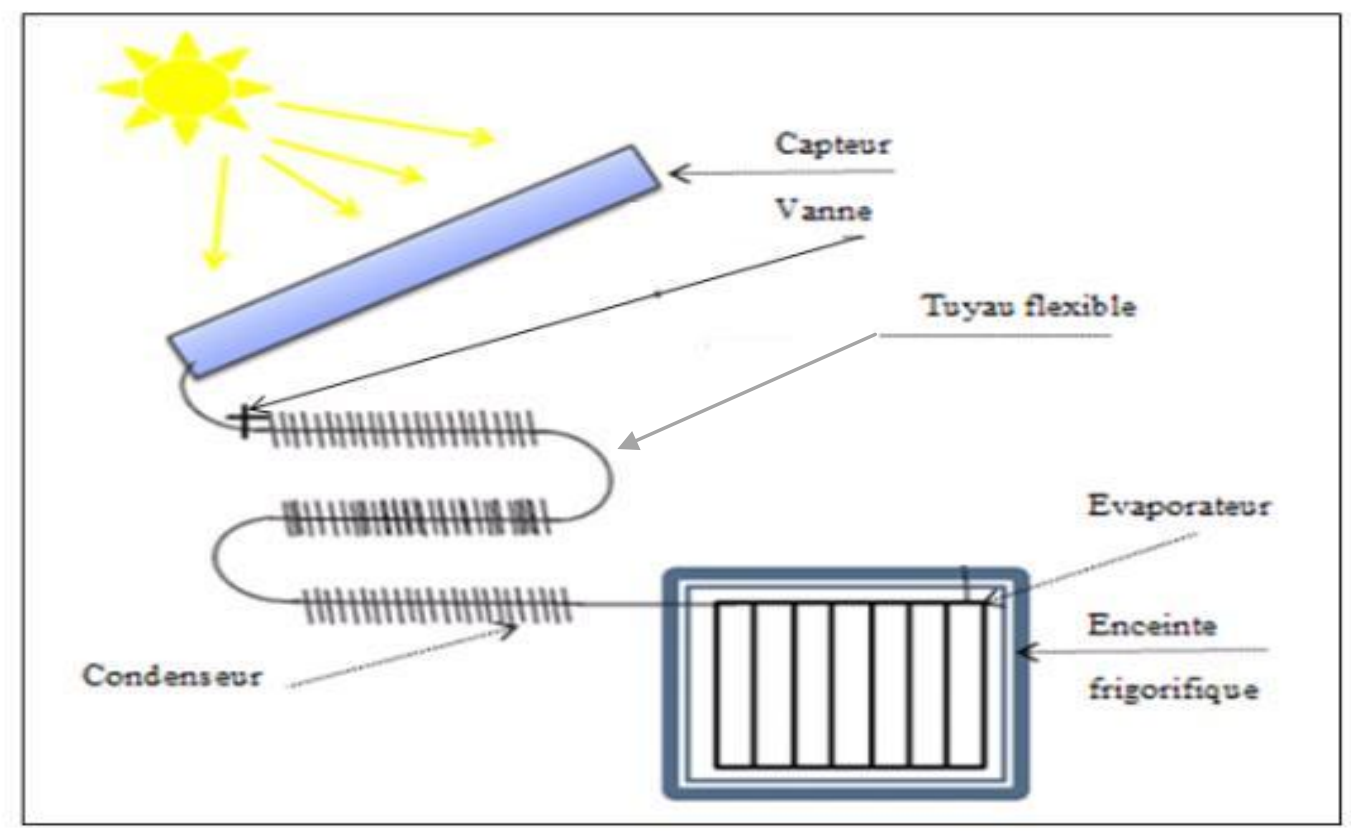

Figure 2.3 : Schéma de principe d'une machine frigorifique solaire à adsorption : phase de chauffage.

\subsubsection{Refroidissement (du $17 \mathrm{~h}$ au $19 \mathrm{~h}$ )}

Lorsque l'ensoleillement diminue, le capteur se refroidit. La pression diminue dans le système jusqu'à la valeur correspondant à la pression d'évaporation à la température de la chambre froide.

\subsubsection{Adsorption- production du froid (du $19 \mathrm{~h}$ au 07h)}

Le système continue son refroidissement, essentiellement par convection naturelle à travers la partie " radiateur » du capteur, et qui fait l'objet de cette étude. L'adsorbant se trouve physiquement en déséquilibre et va se recharger en adsorbant la vapeur continue dans le système.

Cette vapeur est produite par évaporation du liquide se trouvant dans l'évaporateur. Cette opération produit l'effet frigorifique désiré. Le liquide en se vaporisant, emprunte de la chaleur au milieu à refroidir (intérieur de la chambre froide) : C'est la phase active de la machine. 


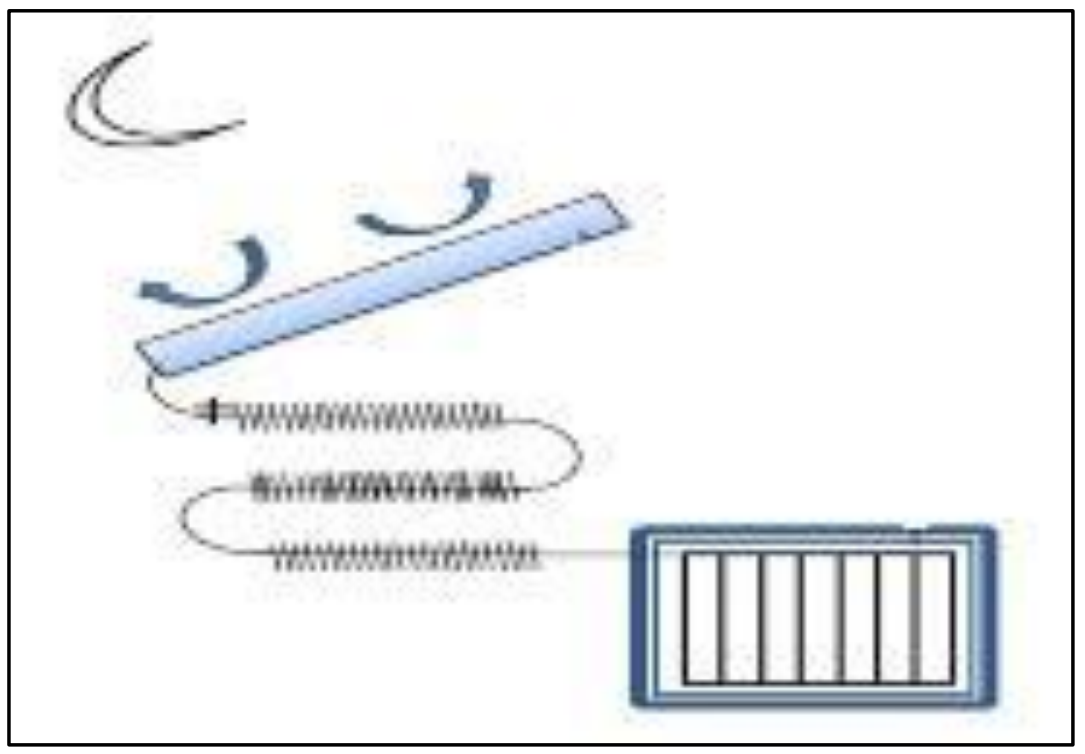

Figure2.4 : Phase de production du froid.

\subsection{Définition des procédés thermochimiques de sorption}

Le principe du procédé thermochimique proposé, pour le type de machine utilisée, repose sur la gestion de la thermicité d'une réaction renversable entre un solide et un gaz :

$$
S_{1}+G \underset{2}{\stackrel{1}{\rightleftarrows}} S_{2}+\Delta H_{r}
$$

Dans le sens 1, appelé synthèse, le gaz $\mathrm{G}$ se fixe sur le solide $\mathrm{S} 1$. Cette réaction chimique est exothermique et libère une quantité de chaleur $\Delta \mathrm{H}_{\mathrm{r}}$. Dans le sens 2, appelé décomposition, lesolide S2 libère du gaz G. Cette réaction endothermique nécessite l'apport de la quantité de chaleur $+\Delta \mathrm{Hr}$.

Le procédé le plus simple de production de froid thermochimique associe un équilibre solide/gaz et un équilibre liquide/gaz. Le dispositif le plus simple est constitué de deux enceintes, l'une contenant le solide réactif, moteur du procédé, l'autre contenant le liquide à évaporer, source de production de froid. Les équilibres correspondants constituent des systèmes monovariants régis par l'équation de Clausius-Clapeyron. Les positions d'équilibres peuvent donc être représentées par des droites dans le diagramme $(\ln (\mathrm{P}),-1 / \mathrm{T})$ de ClausiusClapeyron (Figure 2.5). Dans ce diagramme, on peut également représenter les différentes phases de fonctionnement du procédé de base. 


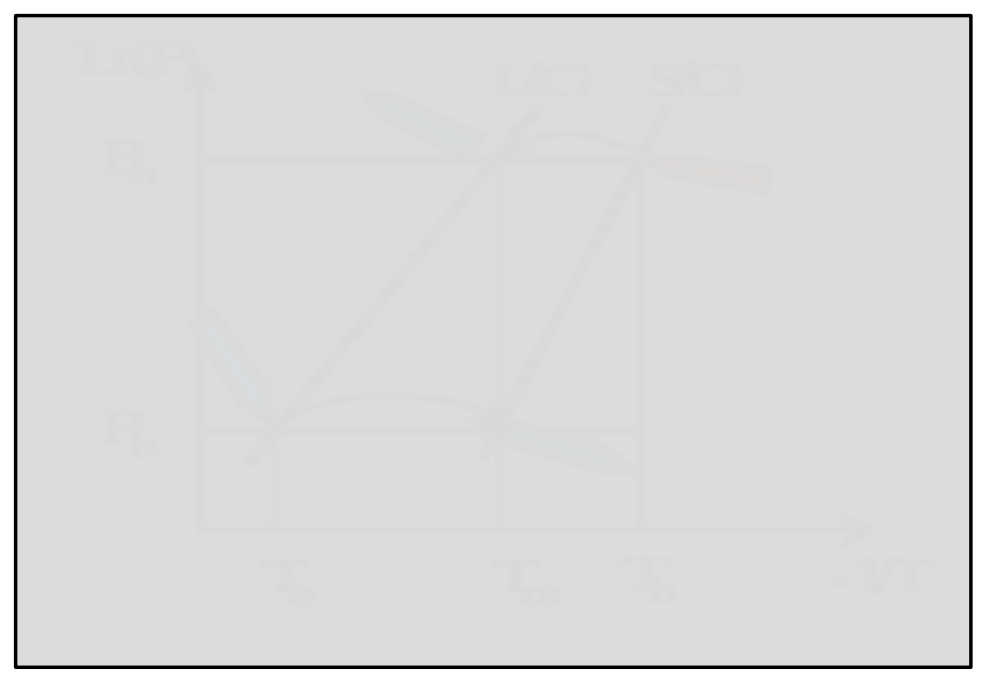

Figure 2.5 : Fonctionnement du système de production de froid par sorption solide/gaz.

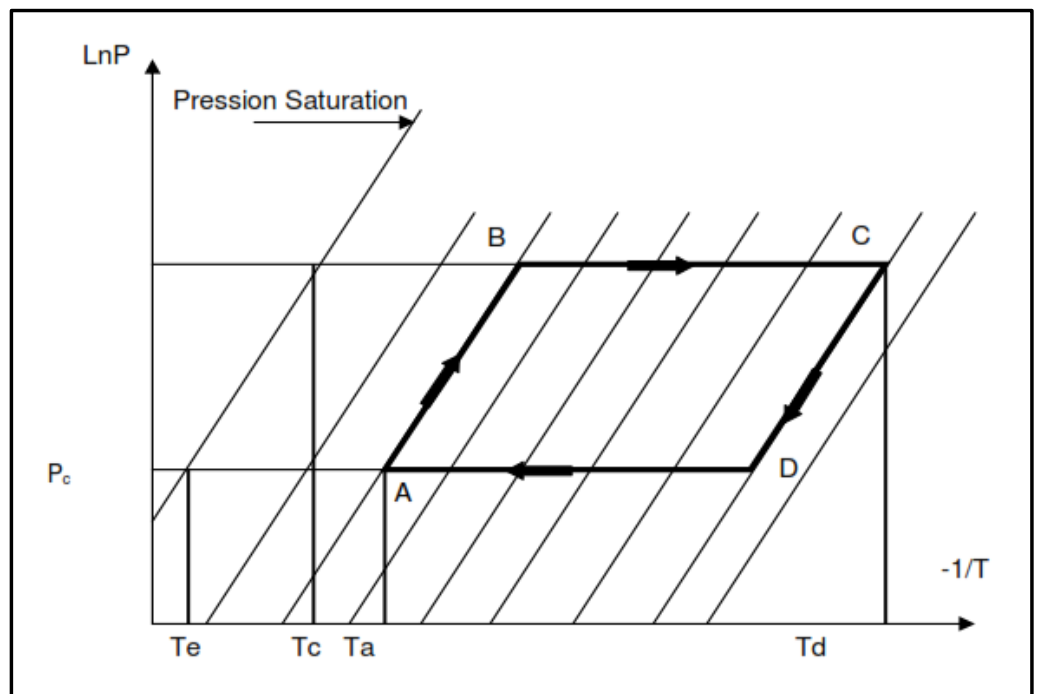

Figure 2.6 : Cycle théorique d'une machine à adsorption.

La Figure 2.6 représente Le cycle théorique d'une machine à adsorption en début de journée, le capteur reçoit le rayonnement solaire (chemin de A à B). L'adsorbant est chauffé, ce qui provoque une élévation de pression (chemin isostérique). Lorsque la pression de vapeur du réfrigérant dans le capteur atteint la valeur correspondant à la température du condenseur, ce dernier est mis en communication avec l'adsorbant (état B).

De $\mathrm{B}$ à $\mathrm{C}$, le capteur est connecté au condenseur. L'apport de chaleur provoque l'augmentation de la température de l'adsorbant et la désorption du réfrigérant. Dans un cycle 
idéal, la pression de condensation reste constante entre $\mathrm{B}$ et $\mathrm{C}$ (isobare). En fin d'après-midi, l'énergie solaire diminue et la température de l'adsorbant atteint son maximum.

La diminution de la température de l'adsorbant (chemin de D à A) qui libère de la chaleur vers l'ambiance provoque une baisse de pression dans le capteur. Lorsque, la pression de vapeur à l'intérieur du capteur est égale à la pression de saturation à la température d'évaporation, le capteur est connecté à l'évaporateur. L'adsorbant, en continuant à se refroidir, « pompe » le liquide réfrigérant dont l'évaporation provoque la production de froid à pression constante.

Le cycle de la machine est dit intermittent car la production du froid se fait uniquement pendant une partie du cycle (la nuit).

Le procédé de congélation solaire proposé ici est donc un procédé thermochimique par nature cyclique. Il alterne une phase de production de froid suivie d'une phase de régénération grâce à l'énergie solaire. La mise en œuvre d'un tel système n'est possible que par l'utilisation de réactions chimiques renversables entre un solide réactif et un gaz couplés à un changement d'état liquide/gaz.

La phase de régénération ayant lieu le jour, l'énergie solaire permet la décomposition du sel contenu dans le réacteur intégré au capteur solaire. Le gaz desorbé est condensé et la chaleur de condensation est rejetée dans un puits de chaleur tel que le milieu ambiant ou le sous-sol. La phase de production de froid a lieu la nuit. L'énergie pompée à la chambre froide permet l'évaporation du fluide de travail. Le gaz produit est absorbé par le sel, dégageant une quantité de chaleur. La chaleur produite par cette absorption peut être évacuée par convection naturelle à travers la partie radiateur du capteur.

\subsection{Thermodynamique de l'adsorption}

\subsubsection{Introduction}

Fontana et Schelle [39] ont découvert le phénomène d'adsorption en 1711. Ce phénomène rend compte de la fixation de molécules de gaz (espèce adsorbable ou adsorbat) sur la surface du solide (adsorbant) au moyen de l'augmentation de la concentration des molécules de ce gaz à la surface du solide par rapport à la quantité totale initiale du gaz. Sans modifier le volume de l'adsorbant. 
Ce phénomène dépend quantitativement des propriétés physiques (porosité, surface spécifique) et chimique (acidité) et de la nature des molécules adsorbées.

Au cours de l'adsorption, le gaz ne subit pas de transformation chimique, le solide conserve son intégrité physique et son architecture cristallographique, en particulier il n'y a pas de gonflement du solide.

Ce phénomène s'accompagne d'un dégagement de chaleur s'appelle chaleur d'adsorption. Il met enjeu deux types d'interactions.

Les interactions adsorbant/adsorbat (entre le solide et les molécules adsorbées);

Les interactions adsorbat / adsorbat (entre les différentes molécules adsorbées).

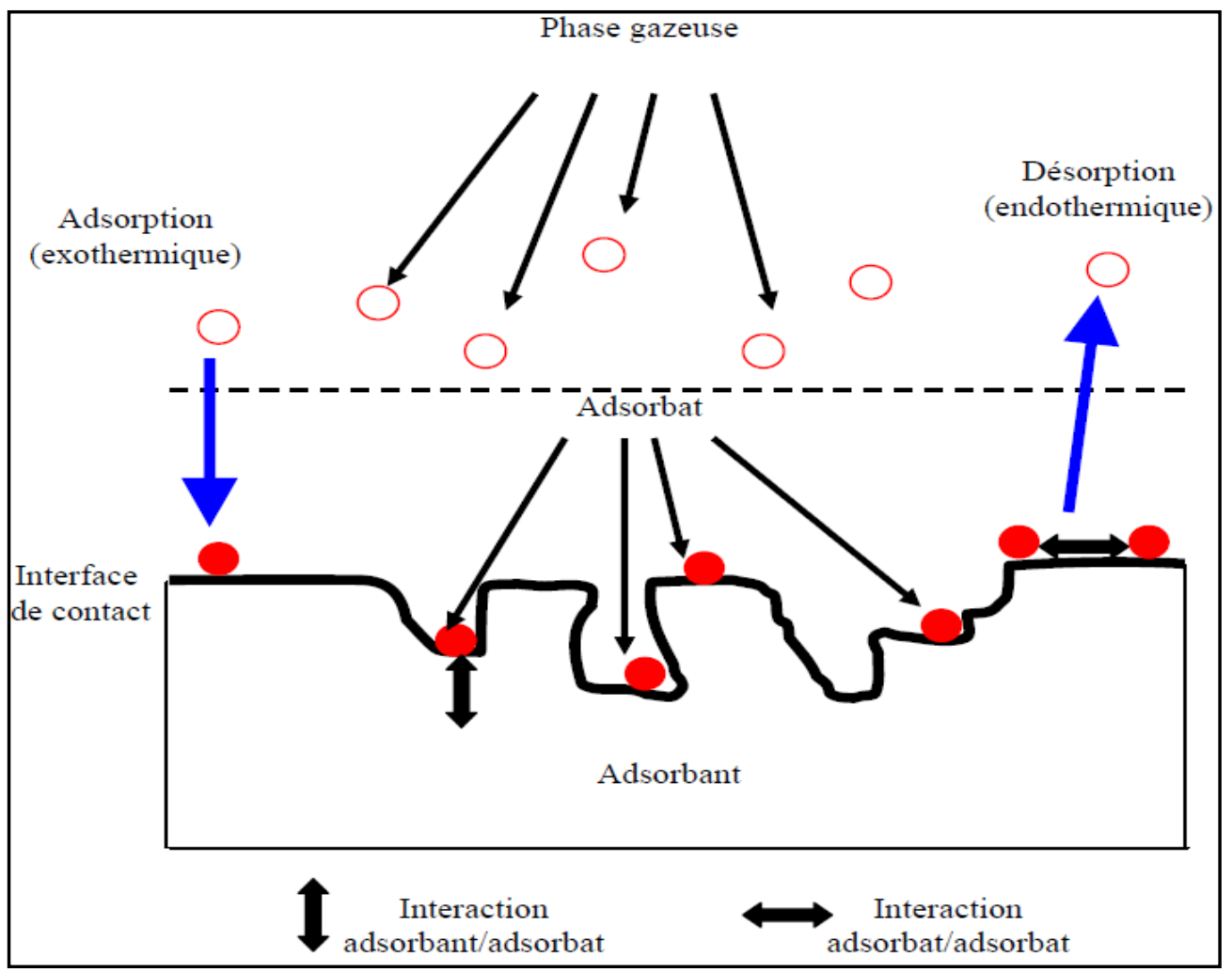

Figure2.7:Phénomène d'adsorption [39].

L'adsorption est déjà utilisée dans un certain nombre de processus physiques et industriels, en particulier dans le domaine de la filtration et de la purification des gaz.de l'affinage des eaux potables et dans les cycles de réfrigération.

L'adsorption d'un gaz par un solide comporte trois phases (Figure 2.7) : 
La phase gazeuse constituée des molécules de gaz;

La phase adsorbée (l'adsorbat) formée des molécules adsorbées à la surface ;

$\checkmark$ La phase solide qu'est l'adsorbant.

Le processus inverse, par lequel les molécules adsorbées sur la surface du solide s'en détachent, notamment sous l'action de l'élévation de la température, se nomme la désorption, ce dernier se traduit par la diminution de la concentration des molécules de gaz à la surface par rapport à la concentration totale du gaz adsorbé [40]. Ce processus est endothermiquecar le solide libère le gaz lorsqu'on lui apporte de la chaleur.

\subsubsection{Types d'adsorption}

L'étude du phénomène d'adsorption montre que la nature de celle-ci varie selon les situations étudiées. Généralement, on distingue deux types d'adsorption qui différent par leur nature de fixation et par les énergies mises en jeu :

\section{a. Adsorptionphysique (physisorption)}

Dans le cas de l'adsorption physique, la fixation des molécules d'adsorbat sur la surface d'adsorbant se fait essentiellement par les forces de liaisons faibles du type Van Der Waalls. Elle se produit sans modification de structure moléculaire, mettant en jeu une chaleur d'adsorption assez faible (de l'ordre de 5 à $25 \mathrm{KJ} / \mathrm{mole}$ ), parfaitement réversible et qui est en outre favorisée par une basse température [39,41].

\section{b. Adsorption chimique (chimisorption)}

Dans le cas de l'adsorption chimique, le processus résulte d'une réaction chimique avec formation des liens chimiques entre les molécules d'adsorbat et la surface de l'adsorbant, donnant naissance à des forces de liaisons très importantes (c'est pourquoi le phénomène est appelé chimisorption). La chaleur d'adsorption mise en jeu est en général assez grande, dépassant les $80 \mathrm{KJ} / \mathrm{mole}$, [39,41]. L'adsorption chimique est souvent irréversible (ou difficilement réversible) et favorisée par une haute température.

La détermination du nombre de degrés de liberté du System consiste en une étape préliminaire dans le développement d'un modèle de simulation, ce nombre représente les variables intensives indépendantes du système, il est déterminé comme suit : 
Lors de l'adsorption d'un gaz sur une surface solide, il y a une distribution des molécules entre les phases gazeuse et adsorbée jusqu'à un état d'équilibre (similaire à celui existant entre un liquide et sa vapeur) dont le nombre de degrés de liberté au sens de GIBBS.

\subsubsection{Relations d'équilibre}

$$
V=n-\varphi+2
$$

Avec : $V$ est le nombre de degrés de liberté, $n$ est le nombre de constituants et $\varphi$ est le nombre de phases.

Donc : $\quad V=2-2+2=2$

Le système est donc bivariant. Cela signifie que deux variables indépendantes seulement décrivent complètement le système et que l'équilibre thermodynamique est contrôlé par ces deux variables. Pour caractériser cet équilibre, nous avons besoin de connaître une grandeur en fonction des deux autres. Il s'agit de la loi décrivant la quantité de matière adsorbée en fonction de la température et de la pression ; donc pour un couple adsorbant/adsorbat donné, la masse adsorbée est donnée par :

$$
\mathrm{m}=f(\mathrm{~T}, \mathrm{P})
$$

Où $f(T, P)$ représente l'approche utilisée pour corréler les variables $T$ et $P$.

On peut constater que l'équilibre peut être décrit de trois façons :

- à pression constante $m=f(T)$, nous obtenons des courbes isobares ;

- à température constante $m=f(P)$, nous obtenons des courbes isothermes.

$\mathrm{Vu}$ ses richesses en renseignements et ses simplicités de réalisation expérimentale, ces courbes sont plus utilisées.

- à masse constante $P=f(T)$,nous obtenons des courbes isostériques; l'expérience et la théorie montrent que ces dernières sont approximativement des droites dans le diagramme de Clapeyron $(\operatorname{Ln}(P),-1 / T)$. 


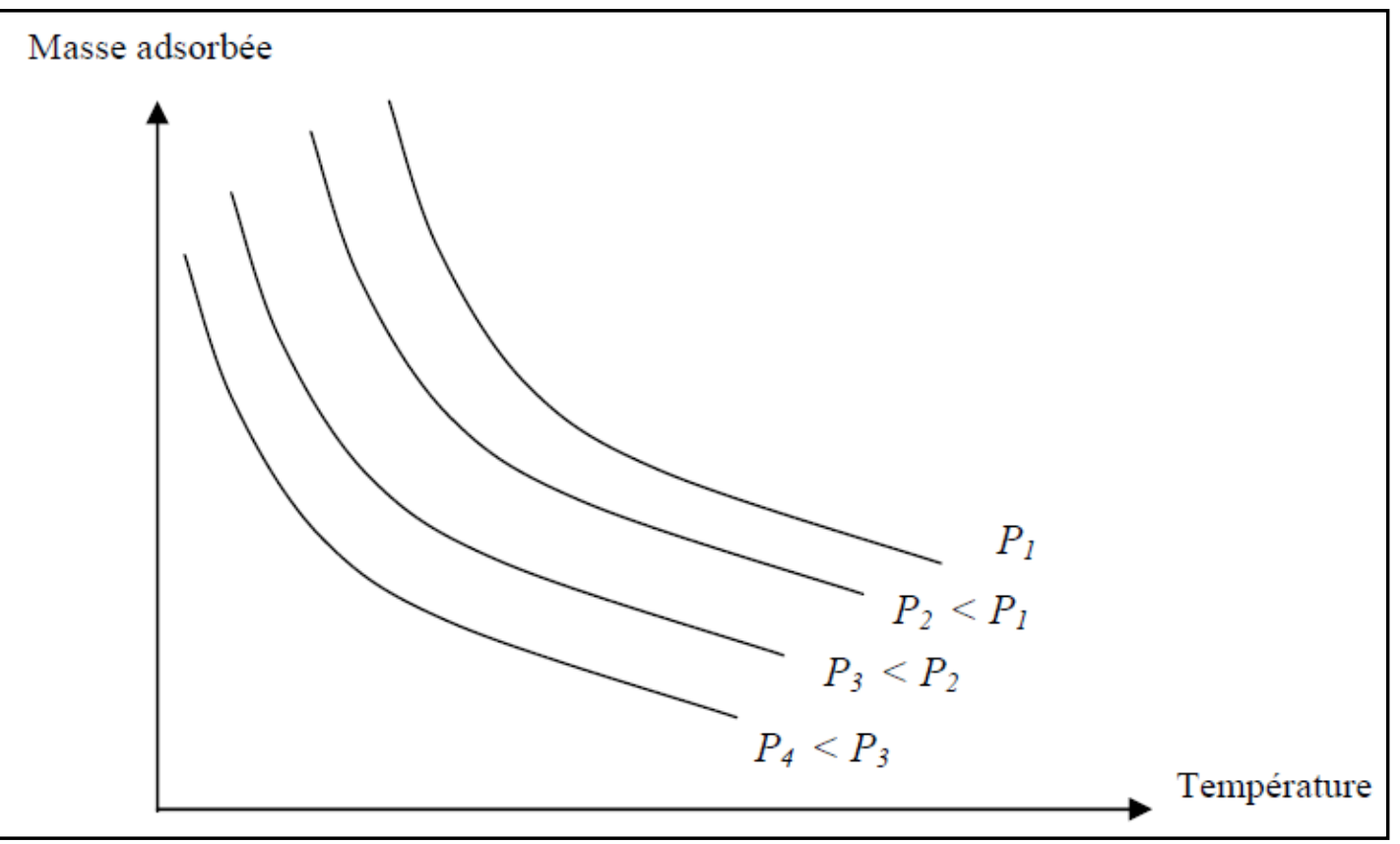

Figure2.8 :Allure schématique des isobares d'adsorption [42].

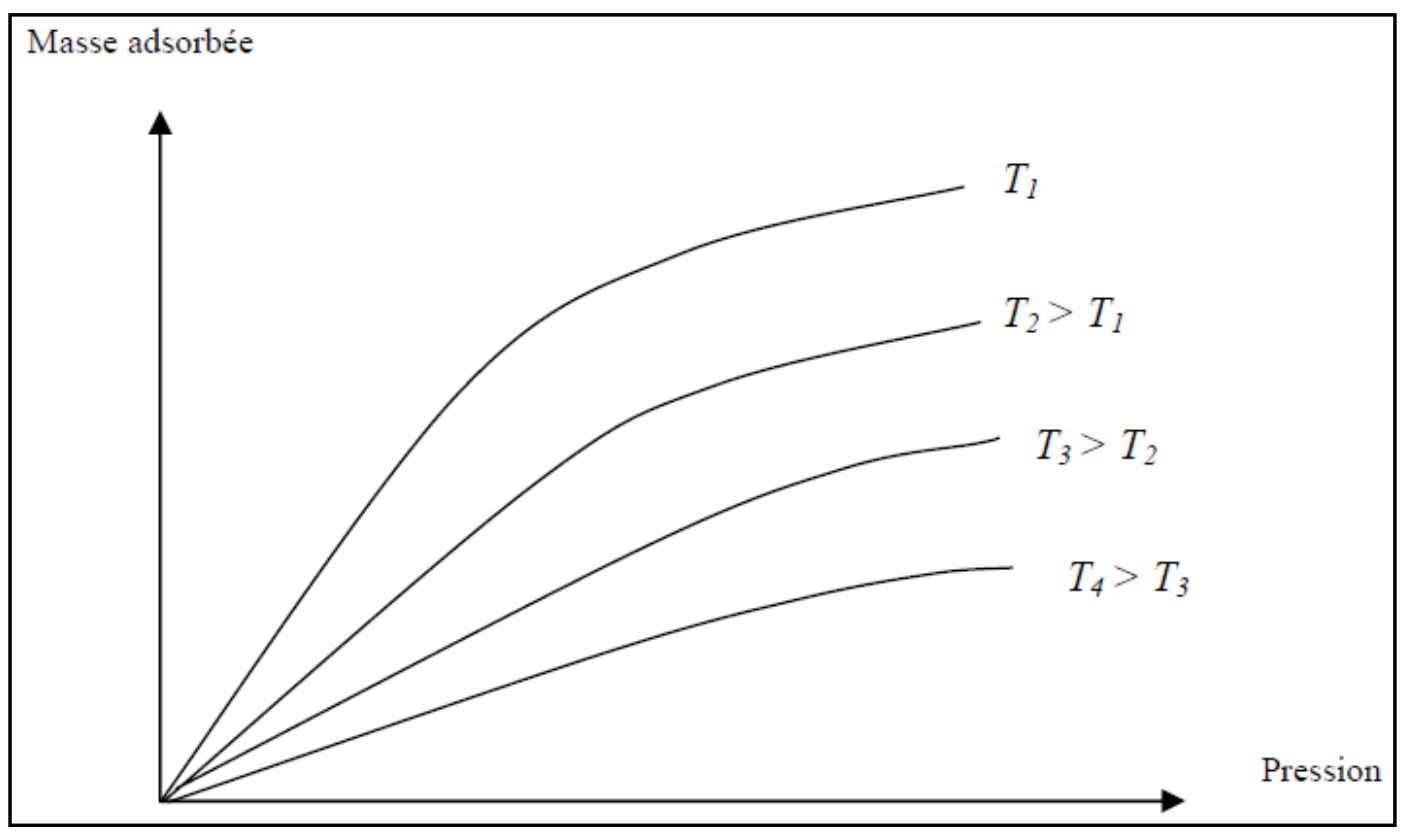

Figure 2.9: Allure schématique des isothermes d'adsorption [42]. 


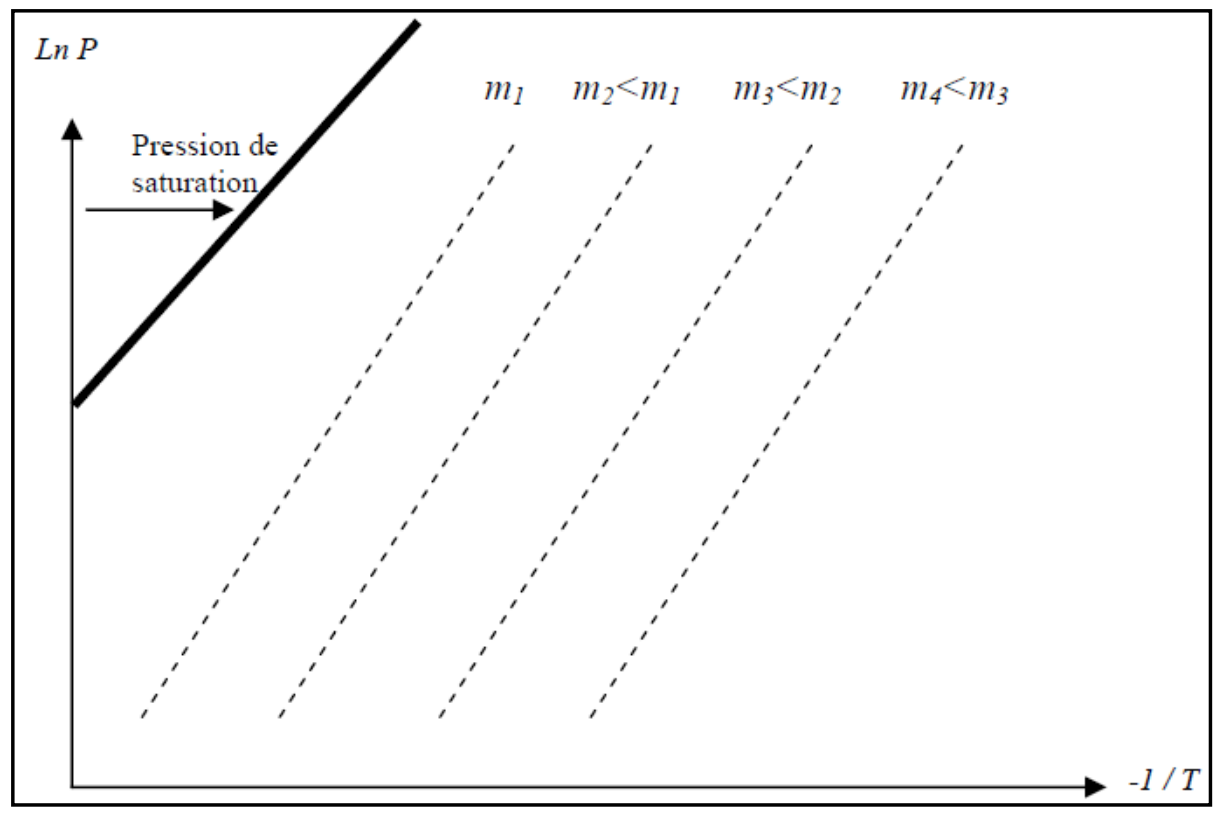

Figure. 2.10 : Schéma d'un réseau d'isostères d'adsorption [42].

\subsubsection{Chaleur isostérique d'adsorption}

L'adsorption est un processus exothermique qui se produit donc avec un dégagement de chaleur ; la principale justification qui a toujours été donnée pour prouver que l'adsorption est exothermique est la suivante :

L'adsorption d'un gaz sur un solide est un processus spontané qui s'accompagne donc d'une diminution d'enthalpie libre de GIBBS du système $(\Delta \mathrm{G}<0)$. En s'adsorbant, le gaz perd desdegrés de liberté. Par conséquent, son entropie diminue $(\Delta \mathrm{S}<0)$ et conformément au deuxième principe de la thermodynamique dont l'équation est :

$$
\Delta H_{a d s}=\Delta G+T \Delta S
$$

Où: $\Delta H_{a d s}$ est l'enthalpie ou chaleur isostérique d'adsorption (appelée aussi chaleur différentielle d'adsorption), cette dernière doit être négative. L'adsorption est donc un processus exothermique.

La chaleur isostérique d'adsorption peut être décomposée en deux termes qui correspondent aux deux étapes habituelles du passage de la phase gazeuse à la phase adsorbée:

Etape 1 : Condensation: $\Delta H=\Delta H_{\text {condensation }}$ 
$\checkmark$ Etape 2 : Fixation des molécules sur la surface active: $\Delta H=\Delta H$ adhésion .

Donc :

$$
q_{s t}=\Delta H_{\text {ads }}=\Delta H_{\text {condensation }}+\Delta H_{\text {adhésion }}
$$

$\Delta H_{\text {condensation }}$ ne fait intervenir que les propriétés de l'adsorbat, tandis que $\Delta H_{\text {adhésion }}$ étant la plus intéressante, est directement liée aux interactions adsorbant/ adsorbat.

Généralement, la chaleur isostérique d'adsorption $q_{s t}$ peut être mesurée par des techniques expérimentales ou estimées à partir de plusieurs méthodes analytiques ; choisissant l'une basée sur les lois de base de la thermodynamique développée comme suit :

Le processus d'adsorption étant un processus à l'équilibre, obéit à une équation du type Clausius-Clapeyron [43]:

$$
\frac{d P}{d T}=\frac{\text { chaleurisostérique d' } \text { adsorption }}{(\text { Vgaz }- \text { Vadsorbat }) T}=\frac{q_{s t}}{(\text { Vgaz }- \text { Vadsorbat }) T}
$$

Comme à l'équilibre, le volume du gaz en phase gazeuse est beaucoup plus grand que le volume de gaz adsorbé, l'équation se réduit à :

$$
\frac{d P}{d T}=\frac{q_{s t}}{V_{g a z}}
$$

Dans le cas où nous avons un gaz parfait, le volume d'une mole du gaz est donné par :

$$
V_{g a z}=\frac{R T}{P}
$$

Cette relation permet de modifier la précédente équation :

$$
\frac{1}{P} \frac{d P}{d T}=\frac{q_{s t}}{R T^{2}}
$$

L'intégration de cette équation permet de trouver :

$$
L n=\frac{-q_{s t}}{R T}+C
$$

$C$ : est la constante d'intégrale. 
La quantité $q_{s t}$ peut dont être déterminée à partir d'une droite représentant la variation de $\ln P$ en fonction de (-1/T), voir la Figure(2.7).

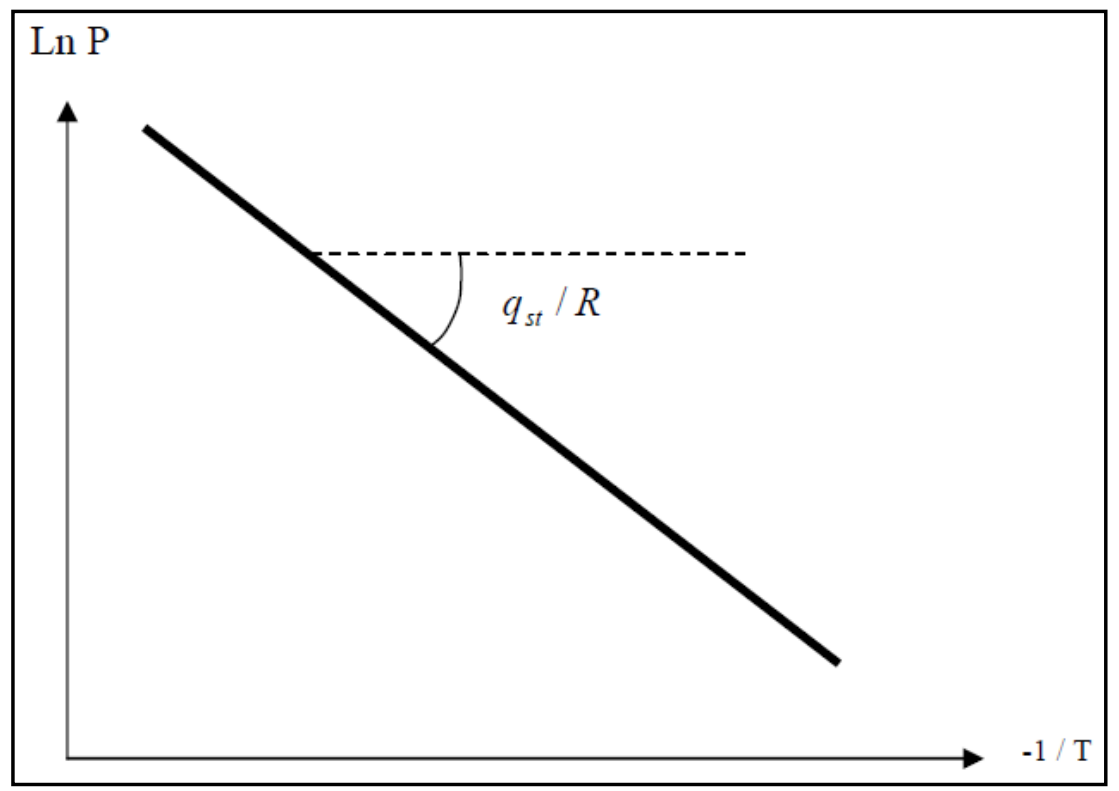

Figure2.11:Détermination de la chaleur isostérique d'adsorption.

\section{Isothermes d'adsorption}

La quantité de réfrigérant $\mathrm{m}_{\mathrm{a}}$ adsorbée à l'équilibre pour une température donnée Test fonction de la pression $\mathrm{P}$ :

$$
m_{a}=f(P)_{T}(2.11)
$$

Les isothermes d'adsorption sont souvent déterminées expérimentalement en utilisant des méthodes gravimétriques, volumétriques ou chromatographiques [44,45]. Ces mesures expérimentales sont ensuite corrélées par des modèles mathématiques qui doivent représenter au mieux les isothermes d'adsorption expérimentales, dans une plage étendue de pression et de températures. En pratique, il est toujours recommandé de rechercher et d'utiliser des modèles ayant à la fois une signification physique, en accord avec les lois fondamentales de la thermodynamique et des facilités de traitement numérique pour les simulations des procédés d'adsorption.

Les isothermes d'adsorption ont des formes variables et peuvent être regroupées en cinq types [46, 47, 48] comme présenté sur la Figure (2.9): 
$\checkmark$ Type I:Sont généralement obtenus dans le cas des adsorbants microporeux avec une saturation progressive des sites d'adsorption sensiblement équivalents.

$\checkmark$ Type II et III :Sont observés avec les adsorbants ayant des distributions larges des tailles de pores avec une transition continue de l'adsorption en monocouche à l'adsorption en multicouche jusqu'à la condensation capillaire.

$\checkmark$ Type IV:Peut résulter de la formation de deux couches successives d'adsorbat à la surface du solide quand les interactions entres les molécules du réfrigérant et la surface solide sont plus fortes que les interactions entre les molécules adsorbées. Dans ce cas, les sites d'adsorption de la seconde couche ne commencent à se remplir que quand la première couche est à peu près complète.

$\checkmark$ TypeV:Contrairement au type IV les forces d'interaction entre les molécules adsorbées sont plus fortes que les forces d'interaction entre les molécules du réfrigérant et le solide.

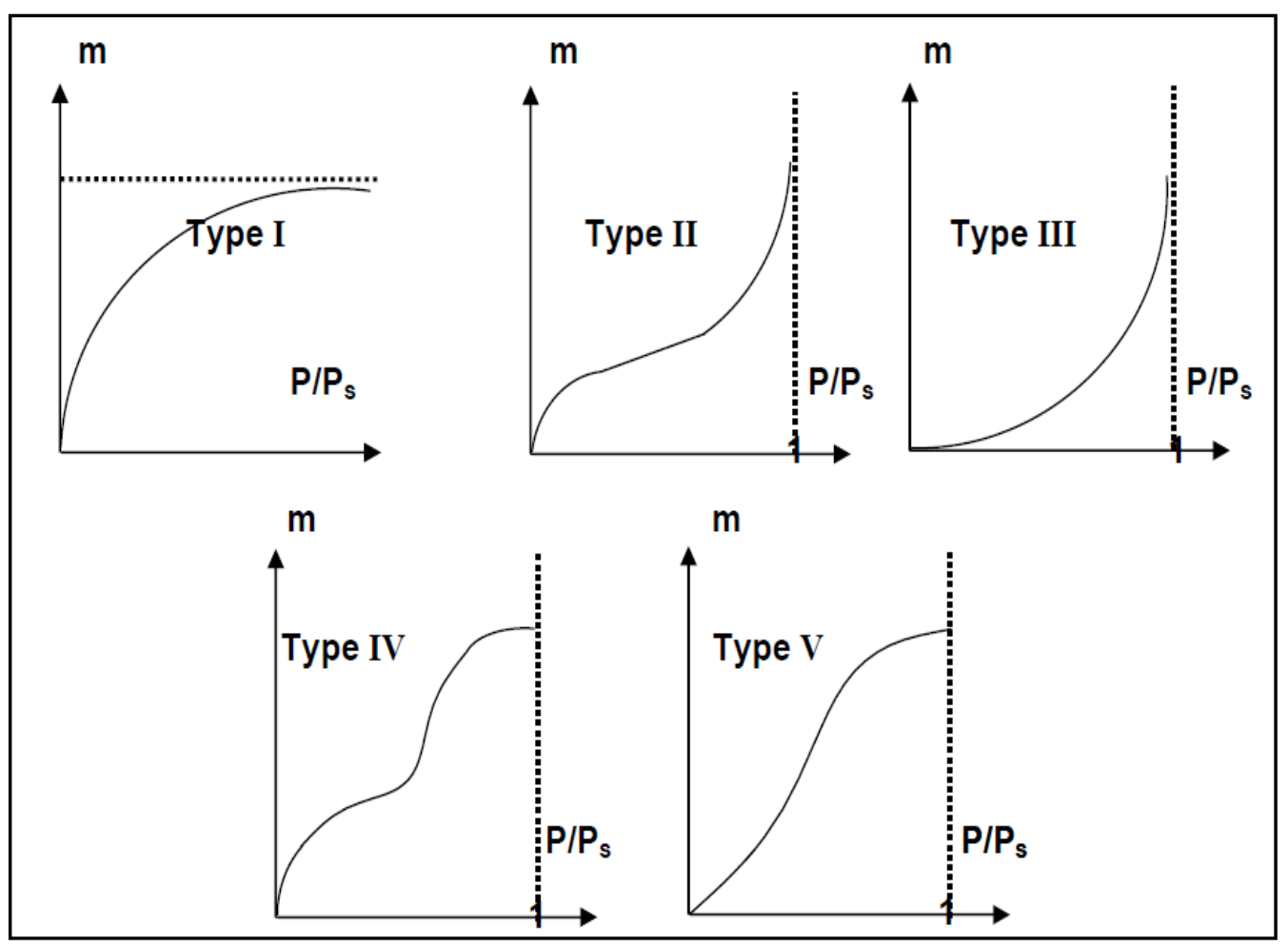

Figure 2.12: les différents types d'isothermes d'adsorption [49].

$\mathbf{P}_{\text {s: }}$ est la pression saturante de l'adsorbat. 
Plusieurs modèles d'isothermes d'adsorption ont été développés, basés soit sur une approche de surface qui considère que l'adsorption est un phénomène de surface, soit sur une approche de volume qui considère que l'adsorption est un phénomène de remplissage de volume. Parmi les approches les plus courantes de la théorie d'adsorption physique citons :

\section{Le modèle de Langmuir}

Langmuir (1916) a développé le modèle d'adsorption monomoléculaire fondé sur des bases théoriques en faisant les hypothèses suivantes :

La chaleur d'adsorption est la même pour toutes les molécules de réfrigérant.

a. L'adsorption se produit en une seule couche c'est-à-dire si une molécule de réfrigérant arrive sur une surface déjà occupée par une molécule adsorbée, elle sera immédiatement réfléchie (Figure 2.8). Il n'y a pas d'interactions entre les molécules adsorbées. Quand le comportement des molécules adsorbées ne dévie pas beaucoup des hypothèses de Langmuir les isothermes d'adsorption sont données par :

$$
q=\frac{m}{m_{0}}=\frac{b P}{1+b P}
$$

Avec :- $q$ est la fraction adsorbée ;

- $m$ : la masse de réfrigérant adsorbée par unité de masse d'adsorbant ;

- $m_{o}$ : la masse de réfrigérant nécessaire pour remplir une couche monomoléculaire par une masse d'adsorbant ;

- $P$ : la pression d'équilibre d'adsorption ;

- $b$ : la constante de Langmuir.

Le paramètre $b$ dépend de la température selon la relation de Van't Hoff

$$
b=b_{0} \exp \left(\frac{Q}{R T}\right)
$$

$Q$ est la chaleur d'adsorption. 


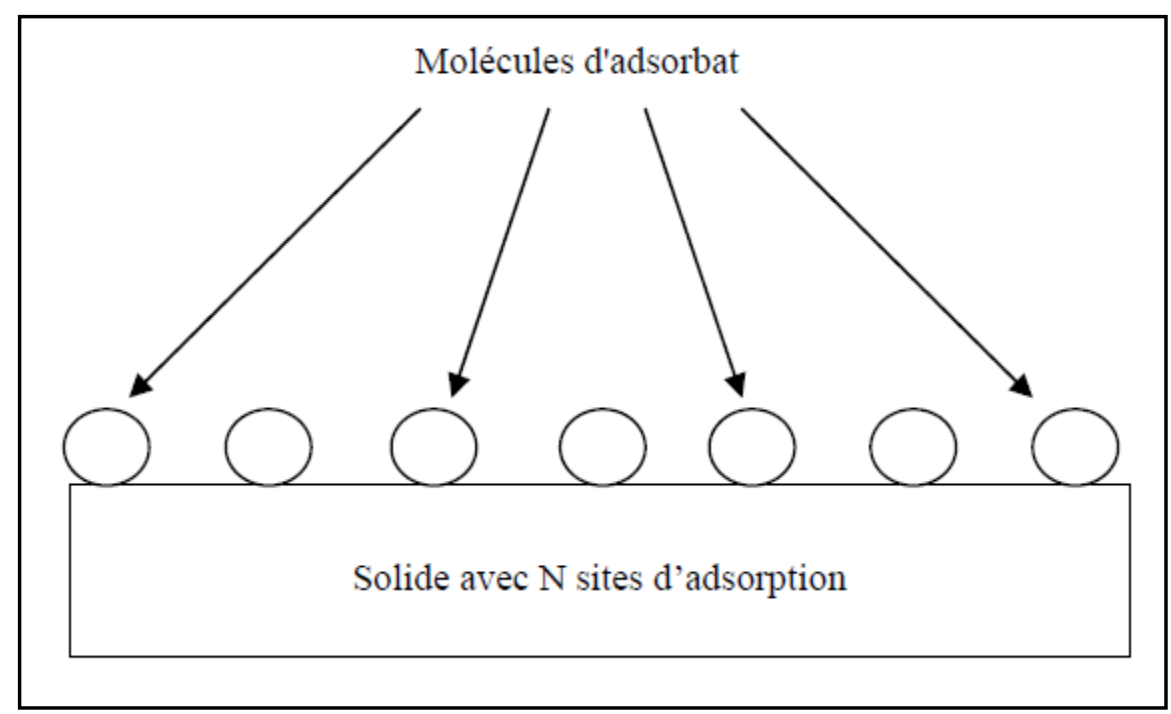

Figure2.13: Modèle d'adsorption en monocouche.

Le modèle de Langmuir représente bien les isothermes d'adsorption de type I.

- Aux basses pressions, $b_{\mathrm{P}}$ est négligeable devant 1 , et $\mathrm{q}$ tend vers $\mathrm{b}_{\mathrm{P}}$, la quantité adsorbée croît linéairement avec la pression : c'est la loi de Henry

- Vers les pressions élevées, 1 est négligeable devant $b_{P}$ et q tend vers 1(la couche est saturée).

- Entre les faibles Pressions où q varie linéairement avec $\mathrm{P}$, et les fortes Pressions où q est égale à 1 , on trouve le modèle empirique de Freundlich où :

\section{$q=K P^{1 / n} \quad(\mathrm{II}-14)$}

Avec K la constante de Freundlich,

$\mathrm{n}$ :une constante caractéristique des interactions entre l'adsorbat et l'adsorbant.

La Fig.II.11résume l'ensemble des expressions de q en fonction de la pression relative. 


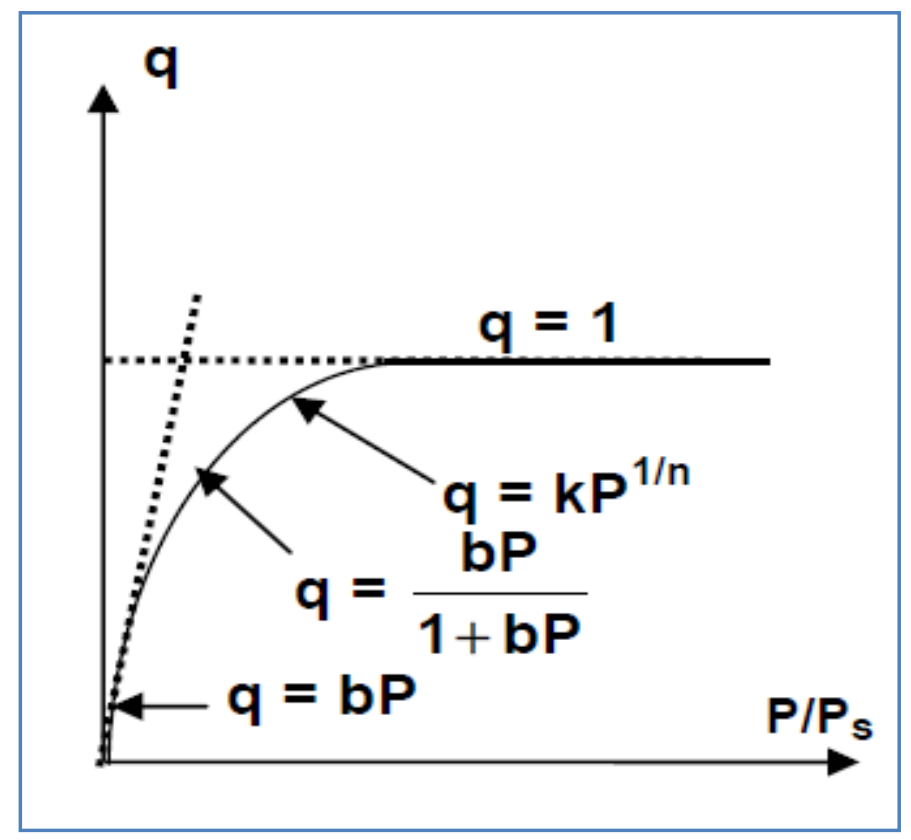

Fig.II.14: Représentation de Henry, Langmuir et de Freundlich de l'adsorption en monocouche

Les isothermes de Freundlich et de Langmuir s'appliquent à la partie arrondie de la courbe $\mathbf{q}=\mathbf{f}(\mathrm{P} / \mathrm{Ps})$. Les deux asymptotes correspondent aux cas limites.

Les isothermes d'adsorption de Langmuir, uniquement valables pour des surfaces énergétiquement homogènes, s'appliquent relativement bien au cas de l'adsorption chimique. Notons qu'à l'origine Langmuir a établi son équation pour le cas de l'adsorption chimique.

Certains solides, par contre, présentent des surfaces hétérogènes où les sites d'adsorption sont de nature différente. Par exemple la structure ionique des zéolithes leur confère une surface hétérogène. Pour les charbons actifs l'hétérogénéité est due aux pores de différentes tailles.

Le modèle de Langmuir- Freundlich permet dans ce cas-là, de représenter les isothermes d'adsorption :

$$
q=\frac{b P^{n}}{1+P^{n}}
$$

Avec $\mathrm{n}$ une constante caractéristique des interactions entre le réfrigérant et l'adsorbant, qui est généralement inférieur à 1 . 
Le modèle de Langmuir-Freundlich se réduit au modèle de Langmuir pour $n=1$.

\section{Modèle de B ET}

Les isothermes d'adsorption de type I c'est-à-dire dont la courbe tend vers une valeur constante (saturation) supposent que l'adsorption se fait en monocouche. Cependant lorsque les forces entre les molécules du réfrigérant et la surface du solide sont suffisamment grande, il y a transition de l'adsorption en monocouche à l'adsorption en multicouche. Langmuir et autres après lui ont essayé d'établir une équation pour les isothermes d'adsorption dans le cas de l'adsorption en multicouches.

Le travail qui a eu le plus de succès est celui de Brunauer, Emmett et Teller(1938), ce travail connue sous le nom de modèle de BET est basée sur une adsorption en multicouche où chacune des couches obéit à la théorie de Langmuir. En effet, quand on étudie les isothermes d'adsorption des gaz à des basses températures sous des pressions pouvant atteindre les pressions de saturation du gaz liquéfié, on obtient des courbes du type II, avec une partie concave (vers l'axe des pressions), aux basses pressions, et au contraire une partie convexe aux hautes pression. Brunauer, Emmett et Teller ont donné une interprétation théorique à l'isotherme type II, en étendant le raisonnement de Langmuir à l'adsorption plurimoléculaires. D'après eux, suivant les points, la surface peut être recouverte de $0,1,2,3, \ldots$, i, .....couches moléculaires de l'adsorbat (Fig.II.12).

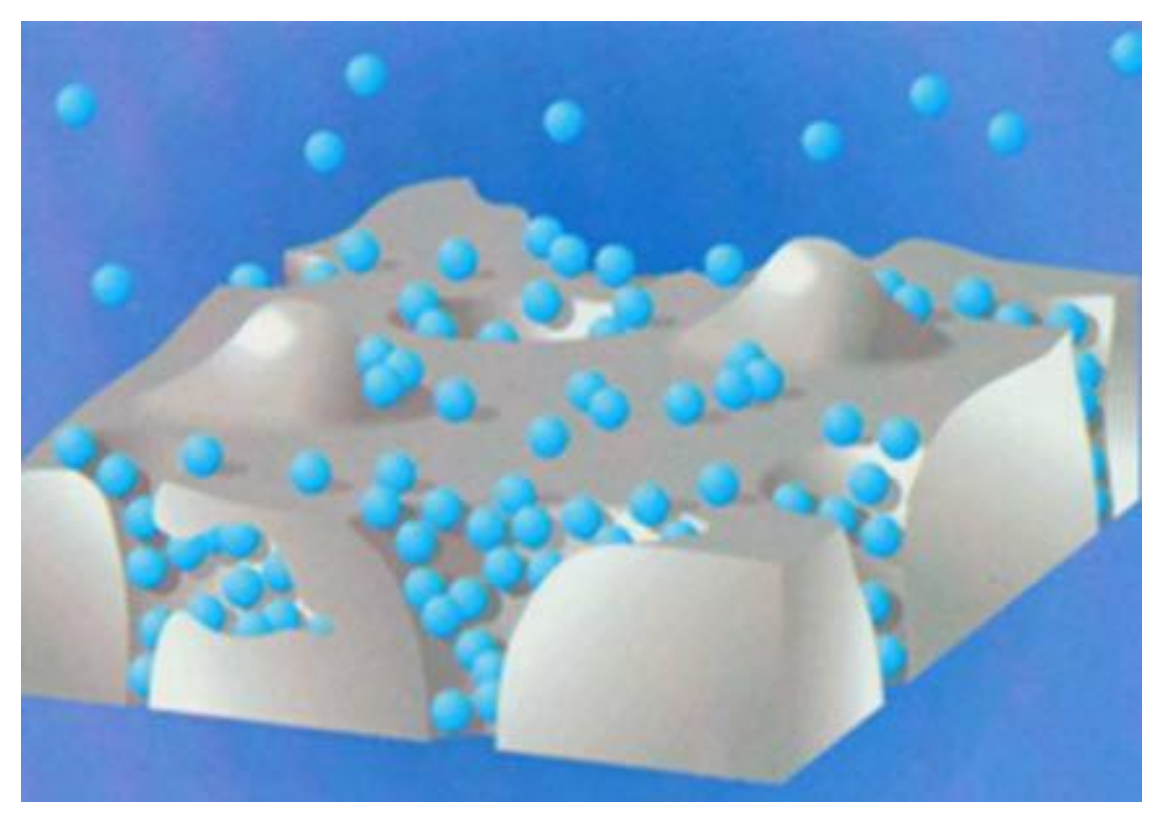

Fig.II.15: Modèle d' adsorption 
Soit:

- $\mathrm{S}_{0} \quad$ la portion de surface inoccupée du solide ;

- $\quad \mathrm{S}_{1}$ la portion de surface occupée par une couche d'adsorbat ;

- $\mathrm{S}_{2} \quad$ la portion de surface occupée par deux couches d'adsorbat ;

- $S_{\mathrm{i}}$ la portion de surface occupée par i couche d'adsorbat ;

- $\quad \mathrm{S}_{\mathrm{n}} \quad$ la portion de surface occupée par n couches d'adsorbat ;

Sur chaque couche, il y a équilibre dynamique entre le nombre de molécules qui s'adsorbent et celles qui se désorbent. On peut donc, sur chaque couche à l'équilibre, admettre que la surface demeure constante. Par exemple, la couche d'ordre 2 se forme par adsorption sur la couche d'ordre un plus la désorption de la couche d'ordre 3. La disparition de cette couche d'ordre 2 se fait par désorption de cette couche plus la formation de la troisième couche.

on suppose ensuite que la vitesse d'évaporation des molécules adsorbées dans une couche d'ordre $\mathrm{j}$ est égale à la vitesse de condensation sur la couche d'ordre $\mathrm{j}$-1, et que seul la formation de la première couche est énergétique, les autres couches subissent une simple condensation. En se basant sur ces hypothèses, Brunauer, Emmett et Teller [50], proposent une équation de forme générale en considérant un nombre fini de couches $\mathrm{n}$ :

$$
q=\frac{m}{m_{0}}=\frac{C\left(P / P_{S}\right)}{1-\left(P / P_{S}\right)}\left[\frac{1-(n+1)\left(P / P_{S}\right)^{n}+n\left(P / P_{S}\right)^{n+1}}{1+(C-1)\left(P / P_{S}\right)-C\left(P / P_{S}\right)^{n+1}}\right]
$$

Avec

- q : la fraction adsorbée

- m : la masse adsorbée par unité de masse d'adsorbant ;

- $\mathrm{m}_{0}$ : la masse d'adsorbat nécessaire pour former une couche monomoléculaire par une masse d'adsorbant ;

- $\quad \mathrm{n}$ : le nombre de couches moléculaires d'adsorbat ;

- C: la constante caractéristique de l'adsorbat, que prend la valeur approximative :

$$
C=\exp \left(\frac{Q_{1}-Q_{L}}{R T}\right)
$$


Avec

- $\mathrm{Q}_{1}$ : est la chaleur d'adsorption de la première couche,

- $\mathrm{Q}_{\mathrm{L}}$ : est la chaleur de liquéfaction, donc de la formation des couches supérieures.

- P: la pression d'équilibre d'adsorption,

- $\quad \mathrm{P}_{S}$ : la pression de vapeur saturante de l'adsorbat à la température considérée.

Pour $\mathrm{n}=1$, l'équation (II-17) se réduit à celle de Langmuir. Pour un nombre infini de couches $(n \rightarrow \infty)$, l'équation (II-17) s'écrit donc :

$$
q=\frac{m}{m_{0}}=\frac{C \frac{P}{P_{S}}}{\left(1-\frac{P}{P_{S}}\right)}\left[\frac{1}{1+(C-1) \frac{P}{P_{S}}}\right]
$$

L'équation Brunauer, Emmett et Teller peut avantageusement être mise sous la forme:

$$
\frac{P}{\left(P_{s}-P\right) m}=\frac{1}{C m_{0}}+\frac{C-1}{C m_{0}} \frac{P}{P_{s}}
$$

Cette forme est commode, car, elle montre que $\frac{\boldsymbol{P}}{\left(\boldsymbol{P}_{\boldsymbol{s}}-\boldsymbol{P}\right) \boldsymbol{m}}$ est une fonction linéaire de $\frac{\boldsymbol{P}}{\boldsymbol{P}_{\boldsymbol{s}}}$

D'ordonnée à l'origine $\frac{1}{C m_{0}}$ et de pente $\frac{(C-1)}{C m_{0}}$.

De cette ordonnée à l'origine et de la pente on déduit aussitôt la valeur de mo, masse du gaz correspondante à la formation d'une monocouche. La théorie de BET a rendu des services précieux en fournissant ainsi un moyen d'estimer la surface spécifique d'un adsorbant. La méthode consiste à déterminer la quantité adsorbée sur la première couche à partir d'une isotherme d'adsorption, généralement obtenue avec l'azote à une température cryogénique $(\mathrm{T}=78 \mathrm{~K})$; la surface des pores est ensuite calculée en supposant que les molécules d'adsorbat recouvrent complètement la surface solide. Ceci constitue l'application la plus importante de l'équation de BET. La linéarité de l'équation BET n'est en général observée que dans un intervalle de pression relative limité est telle que :

$$
0.05<\frac{P}{P_{S}}<0.35
$$

Le modèle de BET est utile pour décrire les systèmes gaz-adsorbant avec condensation capillaire dans les pores dont la distribution des tailles conduit généralement à des isothermes d'adsorption de type II ou III. 


\section{Modèles thermodynamique de Dubinin-Polanyi}

Il s'agit de modèles où le concept de recouvrement en surface, dans le modèle moléculaire, est remplacé par la notion de remplissage en volume des pores notamment pour les adsorbants contenant un grand nombre de micropores avec l'application de la théorie du potentiel de Polanyi [51]. Avec cette approche, on considère que les interactions entre les molécules du réfrigérant et la surface d'adsorbant sont déterminées par un champ de potentiel et que levolume d'adsorbat $\mathrm{W}$ est uniquement une fonction du potentiel de ce champ $\varepsilon$ :

$$
\begin{aligned}
& W=f(\varepsilon) \\
& \text { Ou } \varepsilon=R T \operatorname{Ln}\left(P_{S} / P\right)
\end{aligned}
$$

Avec - $P_{S}$ la pression saturante,

- P la pression d'équilibre,

Le modèle empirique proposé par Dubinin-Radushkevich est donné par

$$
W=W_{0} \exp \left(-K \varepsilon^{2} / \beta^{2}\right)
$$

Avec - W le volume adsorbé par unité de masse d'adsorbant,

- $\quad W_{\mathrm{o}}$ le volume maximale adsorbable par unité de masse d'adsorbant,

- $\mathrm{k}$ une constante,

- $\quad \beta$ un paramètre de normalisation.

Un autre modèle utilisant la même approche est proposé par Dubinin-Astakhov.qui introduit un paramètre supplémentaire $\mathrm{n}$ lié à la distribution des tailles de pores :

$$
W=W_{0} \exp \left(-\frac{K \varepsilon^{n}}{\beta^{n}}\right)
$$

La masse adsorbée par kilogramme dadsorbant à la température T et à la pression P est donnée par :

$$
m(T, P)=\rho(T) W
$$

$\rho(T)$ est la masse volumique de la réfrigérante prise approximativement égale à celle du liquide à la même température. 


\section{Chapitre III :}

\section{Etude du capteur régénérateur de la}

\section{machine frigorifique solaire à}

\section{adsorption}

\subsection{Introduction}

Le procédé de congélation solaire proposé dans cette thèse, est un procédé thermochimique par nature cyclique. Il alterne une phase de production de froid suivie d'une phase de régénération grâce à l'énergie solaire.

La mise en œuvre d'un tel système n'est possible que par l'utilisation de réactions chimiques renversables entre un solide réactif et un gaz couplés à un changement d'état liquide/gaz. La phase de régénération ayant lieu le jour, l'énergie solaire permet la décomposition du sel contenu dans le réacteur intégré au capteur solaire. Le gaz desorbé est condensé et la chaleur de condensation est rejetée dans un puits de chaleur tel que le milieu ambiant ou le sous-sol.

La phase de production de froid a lieu la nuit. L'énergie pompée à la chambre froide 
permet l'évaporation du fluide de travail. Le gaz produit est absorbé par le sel, dégageant une quantité de chaleur. La chaleur produite par cette absorption peut être évacuée par convection naturelle à travers la partie radiateur du capteur. C'est cette dernière partie, "dissipation de la chaleur pendant la nuit", qui fait l'objet de ce chapitre.

La faisabilité du projet de froid solaire est essentiellement basé sur la capacité des capteurs, d'une part, à emmagasiner suffisamment d'énergie durant la journée ceci afin de desorber le gaz et, d'autre part, à évacuer suffisamment la nuit d'adsorber le gaz et en conséquence produire le froid.

L'objectif à remplir durant le jour peut facilement être atteint avec des capteurs conventionnels. Cependant assurer l'évacuation de la chaleur durant la nuit est beaucoup plus délicat. L'objectif est de concevoir un capteur performant le jour, et possédant une fonction de radiateur la nuit. [31]

En cycle nocturne l'énergie à évacuer est de $12 \mathrm{Kw}$ en 5 heures [31], ce qui correspond à une puissance de $2.5 \mathrm{kw}$. Choisissant une surface raisonnable du capteur de 10 $\mathrm{m}^{2}$, la densité de flux à évacuer est de $250 \mathrm{~W} / \mathrm{m}^{2}$. Soit un coefficient d'échange apparent de l'ordre de $25 \mathrm{Wm}^{-2} \mathrm{~K}^{-1}$.

On choisira, dans un premier temps, une plaque plane verticale sur laquelle va se développer l'écoulement de convection naturelle avec une hauteur de $1 \mathrm{~m}$. Elle sera inclinée d'un angle $\alpha$ par rapport à la verticale pour améliorer le rendement de captation en cycle diurne.

A partir des lois de convection naturelle sur une plaque plane verticale en régime laminaire, il est possible d'évaluer le coefficient d'échange résultant, le nombre de Nusselt bâti sur la hauteur $\mathrm{H}$ de la plaque s'exprime par la relation :

$$
\mathrm{Nu}_{\mathrm{H}}=\mathrm{h}_{0} \mathrm{H} / \lambda=0.59 \mathrm{Ra}_{\mathrm{H}}{ }^{1 / 4}
$$

$\boldsymbol{R} \boldsymbol{a}_{\boldsymbol{H}}$ est le nombre de Rayleigh bâti sur la hauteur H :

$$
\mathrm{Ra}_{\mathrm{H}}=\frac{\mathrm{g} \beta \cos \alpha \Delta \mathrm{TH}^{3}}{(\alpha v)}
$$


Les propriétés thermo physiques de l'air sont prises à la température moyenne entre celle de la plaque et celle de l'air à l'infini. Ceci équivaut à avoir un nombre de Rayleigh pour une plaque plane verticale de $1.1410^{9}$.

L'écoulement est donc laminaire à l'intérieur de la couche limite développée.

Le coefficient d'échange $\mathrm{h}_{0}$ résultant prend alors dans ces conditions la valeur :

$$
\mathrm{h}_{0}=2.7 \mathrm{~W} \mathrm{~m}^{-2} \mathrm{~K}^{-1}
$$

La valeur du coefficient d'échange calculée $h_{0}$ est largement inférieure à la valeur $h_{a p p}$ souhaitée (1/10 environ). Une autre solution autre que la plaque plane s'impose pour le dopage des échanges convectifs et qui prendra en considération les performances du capteur en période diurne.

La solution qui été retenue est celle d'un capteur muni d'ailettes rectangulaires sur sa partie basse, (fig. III.1).L'échange se fera exclusivement par convection naturelle [32], Le problème consiste alors à bien dimensionner ces ailettes avec une forme, un écartement et une épaisseur optimale afin de dissiper la quantité voulue de chaleur.

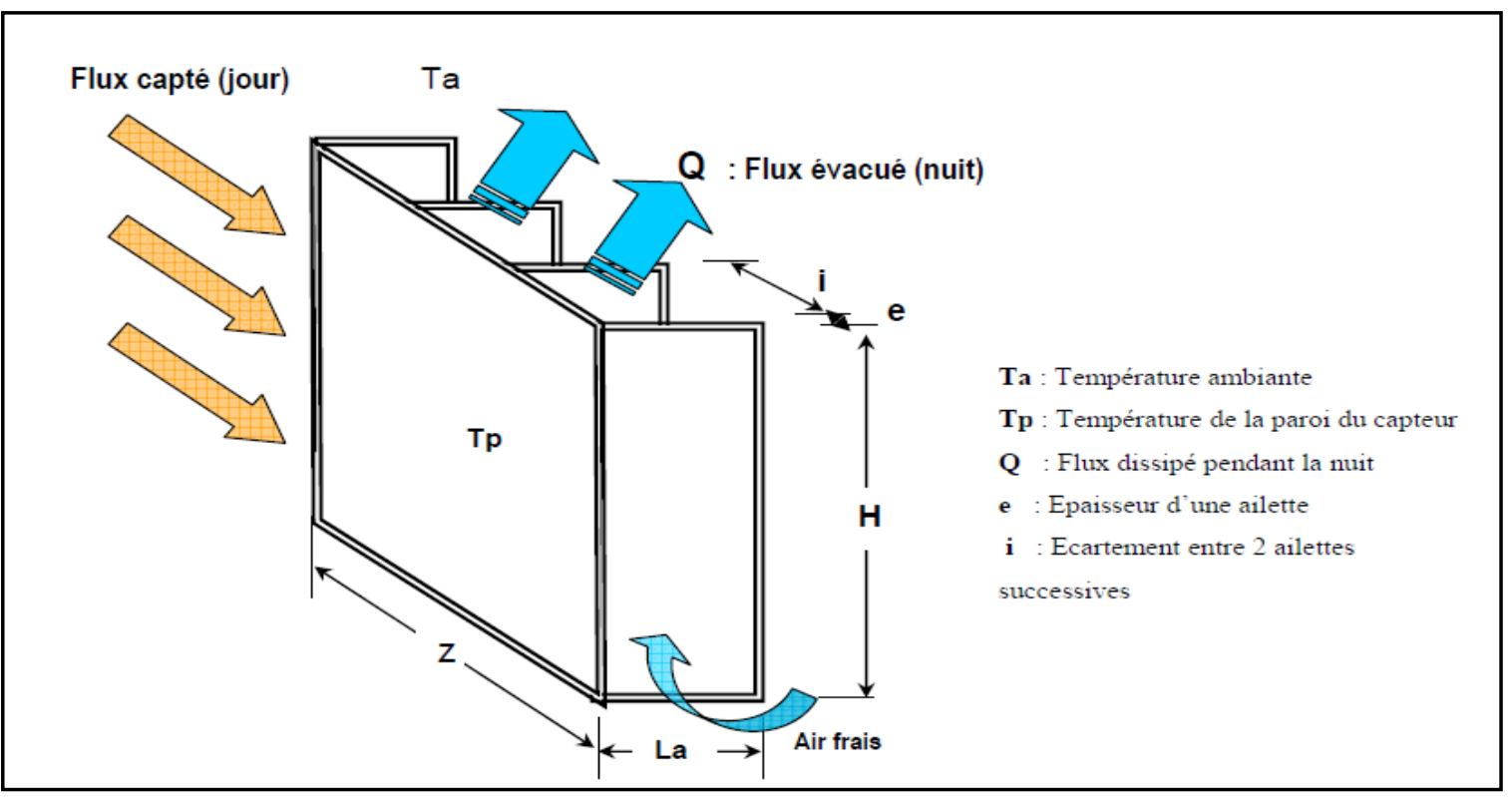

Fig.III.1: ailette et longueurs caractéristiques

\subsection{Etude théorique}

Considérons une ailette d'épaisseur e, de longueur La, de largeur H, l'épaisseur est supposée être petite par rapport à la longueur et à la largeur. On néglige toutes les variations 
de températures sur une section droite de l'ailette, et on suppose que $\mathrm{T}$ est en fonction de la seule distance x par rapport à la paroi

On pose :

$\mathrm{T}_{\mathrm{F}}:$ Température de l'air

$\mathrm{T}_{0}$ : Température de la paroi, et donc de l'ailette en $\mathrm{x}=0$

$\lambda$ : Conductivité thermique de l'ailette

h: Coefficient d'échange moyen entre l'ailette et l'air.

Considérons le petit volume en forme de parallélépipède de largeur $\mathrm{H}$, d'épaisseur e et de longueur $\mathrm{dx}$ :

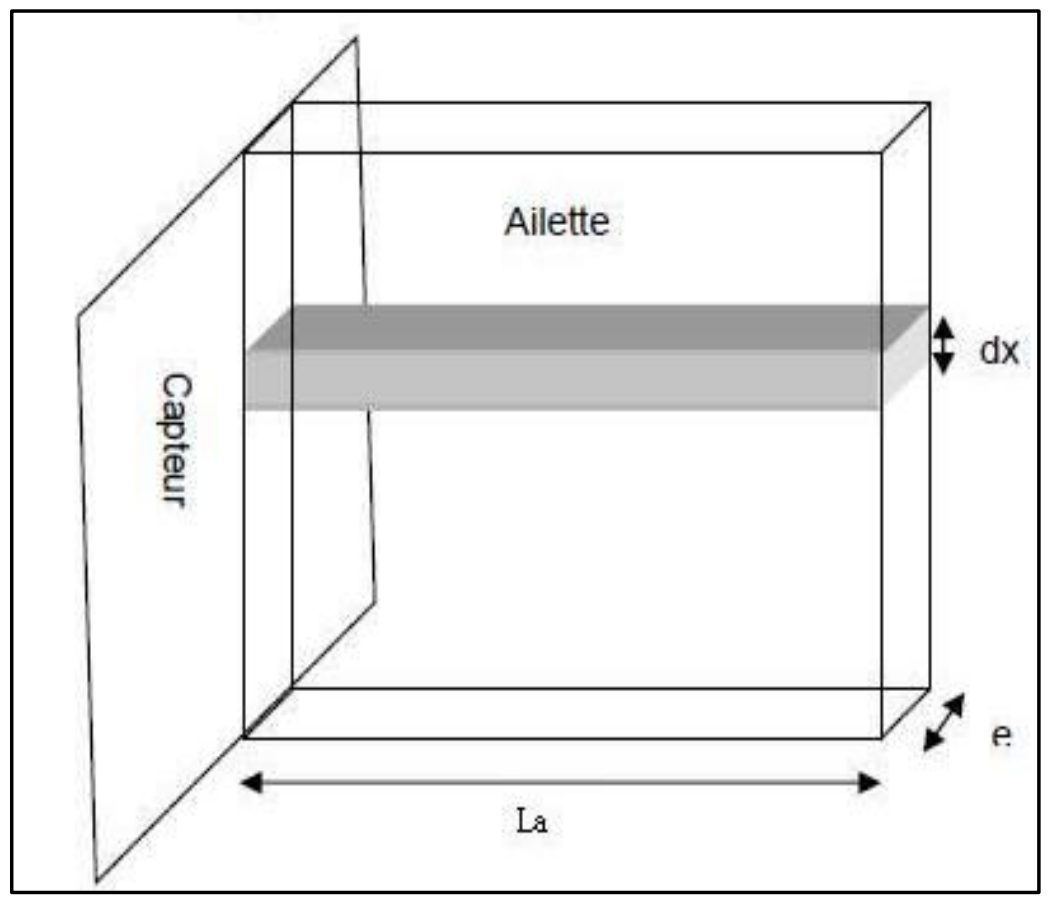

Fig. III.2: équation d'ailette

Sur ce petit volume, le bilan thermique s'écrit :

$$
\begin{aligned}
& \varphi_{x} \Omega-\varphi_{x+d x} \Omega-\mathrm{hs}\left(T_{x}-T_{F}\right)=0 \\
& \varphi_{x+d x}=\varphi_{x}+\frac{\partial \varphi_{x}}{\partial x} d x \quad \text { et } \quad \varphi_{x}=-\frac{d T_{x}}{d x}
\end{aligned}
$$

- Il reçoit de la chaleur par conduction, du côté du mur, sur une surface $\mathrm{W}=\mathrm{H} \cdot \mathrm{e}$.

- Il cède de la chaleur par conduction par la face opposée, sur la même surface $\mathrm{W}$.

- Il cède de la chaleur par convection sur une surface $S=P . d x$ ou $\mathrm{P}=2 *(\mathrm{H}+\mathrm{e}) ; \mathrm{P}$ est le périmètre. 


$$
\begin{gathered}
\varphi_{x}-\varphi_{x+d x}=-\frac{\partial \varphi_{x}}{\partial x} d x \quad \text { et } \frac{\partial \varphi_{x}}{\partial x}=-\frac{d^{2} T_{x}}{d x^{2}} \\
{\left[-\left(-\frac{d^{2} T_{x}}{d x^{2}}\right) d x\right] \Omega-\operatorname{hpdx}\left(T_{x}-T_{F}\right)=0}
\end{gathered}
$$

Ce qui donne $\frac{\lambda \Omega}{h p} \cdot \frac{d^{2} T_{x}}{d x^{2}}-\left(T_{x}-T_{F}\right)=0$

On suppose $\theta=\left(T_{x}-T_{F}\right)$

On obtient $\quad \cdot \frac{d^{2} \theta}{d x^{2}}-m^{2}=0$

On suppose que la température est uniforme le long de la direction verticale de l'ailette. Le nombre total d'ailettes à implanter est $\mathrm{N}$ avec :

$$
\mathrm{N}=\frac{\mathrm{Z}}{\mathrm{e}+\mathrm{i}}
$$

Le coefficient d'échange apparent est :

$$
\mathrm{h}_{\mathrm{app}}=\frac{\mathrm{Q}}{\left[\mathrm{T}_{\mathrm{P}}-\mathrm{T}_{\mathrm{a}}\right] \mathrm{ZH}}
$$

Q étant le flux total dissipé :

Le coefficient d'échange apparent s'exprimera, d'après l'étude théorique [33], alors par

$$
h_{\text {app }}=\frac{z \cdot i \cdot H}{e+i} \frac{h}{z \cdot H}+\frac{2 N L a H h \eta}{z \cdot H}
$$

Le coefficient réel d'échange h est calculé à partir de la corrélation :

$$
N u_{i}=\frac{h . i}{\lambda}=\frac{1}{\sqrt{\frac{576}{\left(R a^{*}\right)^{2}}+\frac{2.873}{\left(R a^{*}\right)^{1 / 2}}}}
$$

Où Ra* est le nombre de Rayleigh corrigé :

$$
R a^{*}=R a_{i} \frac{i}{H}
$$

De plus et pour faciliter les calculs, on définit les longueurs adimensionnelles de l'ailette :

$$
\text { Ecartement adimensionnel } x=\frac{i}{H} \sqrt[4]{R a_{H}}
$$


Epaisseur adimensionnelle $\quad e^{*}=\frac{e}{H} \sqrt[4]{R a_{H}}$

Longueur adimensionnelle $L a^{*}=\frac{L a}{H} \sqrt[4]{R a_{H}}$

L'équation (III-8) devient alors :

$\frac{h H}{\lambda_{a}}=\frac{x \sqrt[4]{R a_{H}}}{\sqrt{\left(\frac{576}{x^{4}}+2.873 x^{2}\right)}}$

Donc le coefficient d'échange apparent de la plaque sera :

$$
\frac{H h_{a p p}}{\lambda_{a}}=\frac{2 L a^{*} \sqrt[4]{R a_{H}}}{\left(1+\frac{e^{*}}{x}\right) \sqrt{\left(\frac{576}{x^{4}}+2.873 x^{2}\right)}}\left(\eta+\frac{x}{2 L a^{*}}\right)
$$

L'équation de la chaleur au sein de l'ailette s'écrit de la manière suivante [34] :

$\frac{d^{2} \theta}{d x^{2}}-m^{2} \theta=0 \theta=T-T_{a} T_{a}$

étant la température de l'air.

Cette équation admet pour solution :

$\theta=\theta_{0} \frac{\cosh (m(L a-x))}{\cosh (m L a)}$

$\theta_{0}=T_{p}-T_{a}$

L'efficacité de l'ailette $\eta$, est définie par [33] :

$\eta=\frac{1}{L a} \frac{\int_{0}^{L a}\left(T-T_{a}\right) d L}{\left(T_{p}-T_{a}\right)}$

Soit

$\eta=\frac{-\left.\lambda H e \frac{d \theta}{d x}\right|_{x=0}}{2 \operatorname{LaHh}\left(T_{p}-T_{a}\right)}=\frac{\tanh [(m L A)}{\mathrm{mLa}}$

Avec: $m \cong \sqrt{\frac{2 h}{\lambda e}}$ et $\lambda$ est conductivité thermique de l'ailette. 


\subsection{Résultat de l'étude théorique}

Le coefficient d'échange est donné par l'équation (III-8).

Le gain correspond à l'implantation des ailettes est donc donné par :

$$
\frac{H h_{a p p}}{h_{0}}=\frac{2 L a^{*} \sqrt{2.873}}{\left(1+\frac{e^{*}}{x}\right) \sqrt{\left(\frac{576}{x^{4}}+2.873 x^{2}\right)}}\left(\eta+\frac{x}{2 L a^{*}}\right)
$$

A partir de cette équation, nous allons évaluer le gain apporté par les ailettes en fonction des caractéristiques géométriques de celles-ci.

\subsubsection{Influence de l'épaisseur de l'ailette}

On remarque que l'augmentation de l'épaisseur de l'ailette engendre une légère diminution de la valeur du gain. Cependant cette influence est relativement faible. On choisi donc la valeur moyenne. On prendra comme valeur optimale $\mathrm{e}^{*}=0.50$ ce qui correspond à une épaisseur effective de l'ailette de $3 \mathrm{~mm}$.

\subsubsection{Influence de l'écartement}

Le gain augmente avec l'écartement et ceci jusqu'à une valeur optimale de x à déterminer puis décroît pour tendre vers 1 .

\subsubsection{Influence de la longueur de l'ailette}

Pour déterminer la valeur optimale de l'écartement, on a représenté le gain en fonction de $\mathrm{La}^{*}$ pour différentes valeurs de x. On voit clairement que la valeur optimale est de 3, ce qui correspond une distance entre deux ailettes successives de $1.6 \mathrm{~cm}$.

On remarquera en plus, que l'évolution du gain est presque linéaire, ce qui signifie que l'ailette transmet de la chaleur par convection sur la totalité sur cette longueur.

Cela correspond à une valeur réelle de la longueur de l'ailette : La<22

Enfin on constate que pour $\mathrm{x}=3$, le gain atteint une valeur de 10 , ceci pour une valeur de $\mathrm{La}^{*}=20$, soit une valeur réelle de l'ailette de $10 \mathrm{~cm}$.

En conclusion à cette étude, on peut dire que la meilleure configuration géométrique pour l'obtention d'un coefficient d'échange apparent de l'ordre de $25 \mathrm{Wm}^{-2} \mathrm{k}^{-1}$ est réalisée avec 
les données suivantes : Epaisseur de l'ailette e $=4 \mathrm{~mm}$, écartement $\mathrm{i}=1.6 \mathrm{~cm}$ et une longueur de l'ailette de $\mathrm{La}=10 \mathrm{~cm}$. Ces paramètres apparaissent encouragent du point de vu réalisation.

Ces résultats rejoignent parfaitement les conclusions de l'étude préliminaire réalisée au LET [35], du point de vue réalisation, ces données paraissent tout à fait réalisables.

Cette étude reste limitée puisqu'elle n'est que théorique. Pour s'assurer de sa validité, nous allons effectuer une simulation numérique 3D pour le dopage des échanges par convection naturelle au niveau du capteur pendant la phase nocturne en utilisant le code FLUENT. Une étude complémentaire sur d'autres formes d'ailettes, autres que rectangulaires, sera un plus à ce travail. 
L'utilisation de données spécifiques au climat algérien donnera une dimension pratique. Pour rappel, les études préliminaires faites au LET ne sont basées que sur le climat européen.
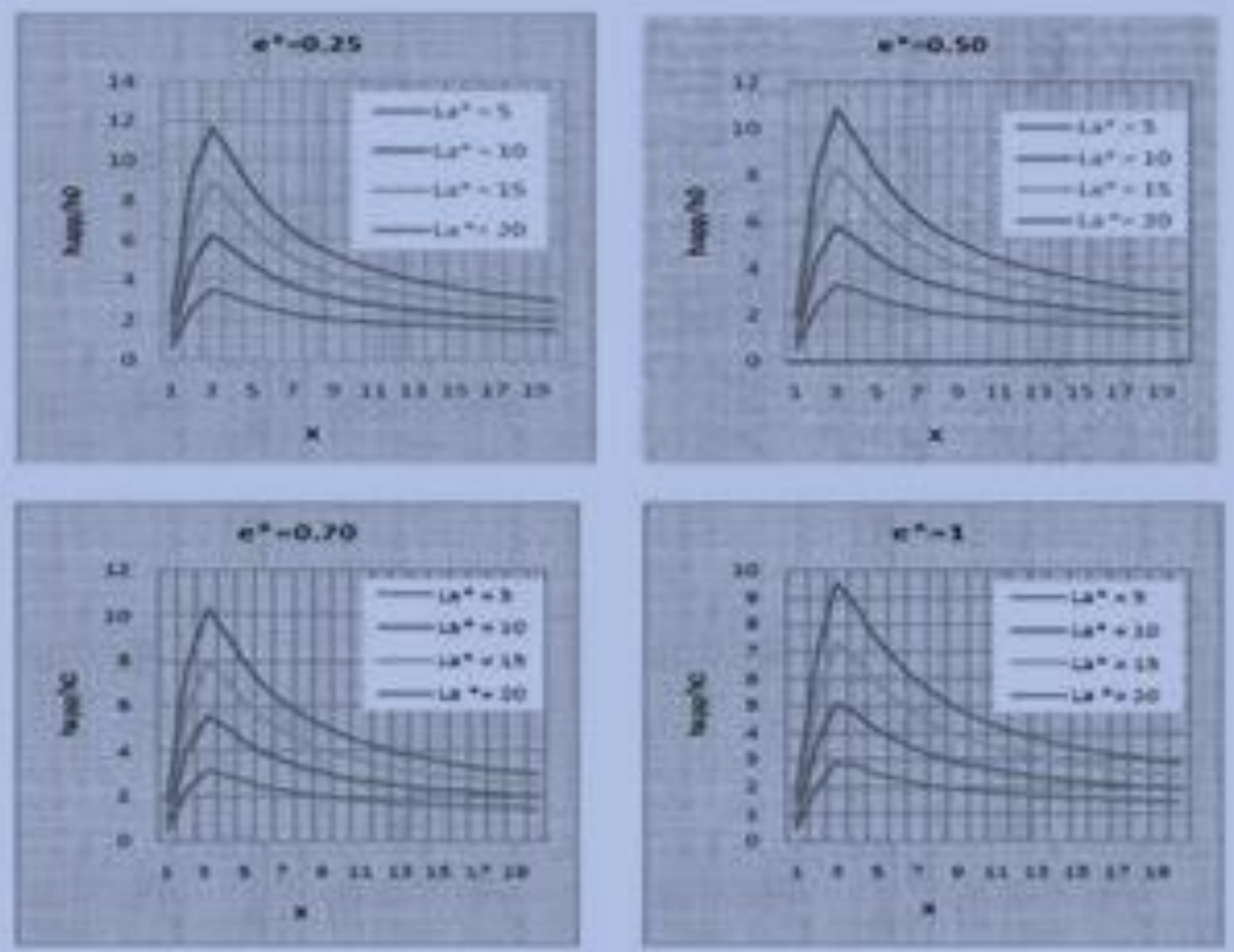

Fig III 3: Influence de e* sur le grin en fonction de l'erpacensent $x$

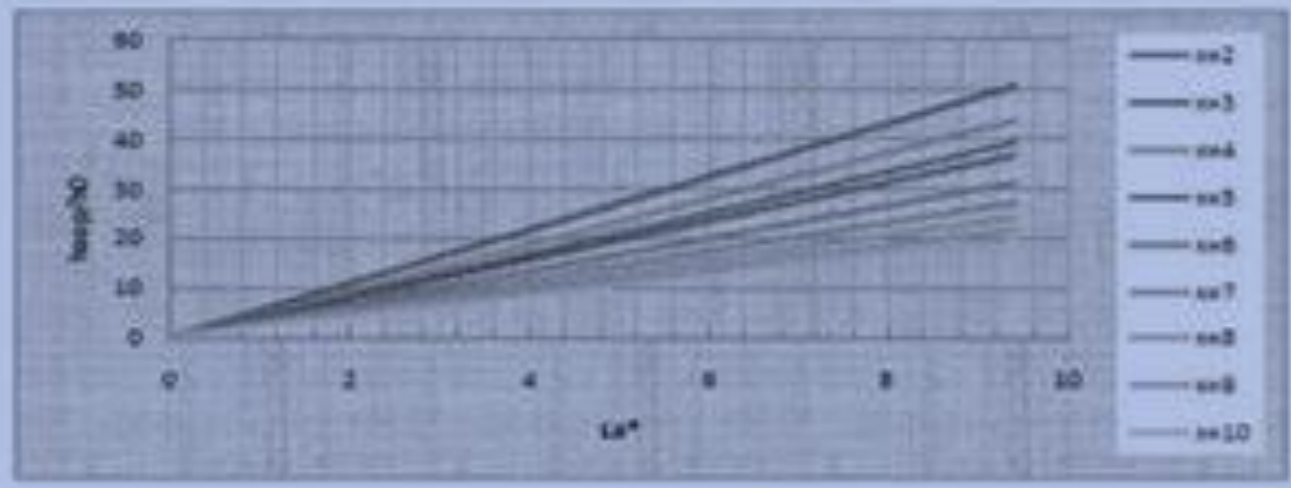

FigIII 4: Calaul du gin en fanction de L $2^{*}$ pour differenter valeuss de $x$. Cas de $e^{*}=0.7$ 


\subsection{Simulations numériques 3D}

\subsubsection{Introduction :}

Dans cette section, on s'intéresse à l'analyse des résultats numériques obtenus en ce qui concerne l'influence des dimensions (géométrie) du capteur sur le comportement d'écoulement d'air, pour ça on va faire une simulation numérique à fin de s'assurer des résultats obtenu dans l'étude théorique par le logiciel Fluent.

\subsubsection{Domaine d'étude}

Le domaine d'étude (capteur +ailettes) présente une périodicité, ainsi des symétries, ce qui permet de le réduire à un motif élémentaire, Il englobe la moitié d'une ailette et un écartement de capteur (distance entre deux ailettes successives). Les conditions aux limites sont connues, le domaine résultant est représenté sur la figure 5.

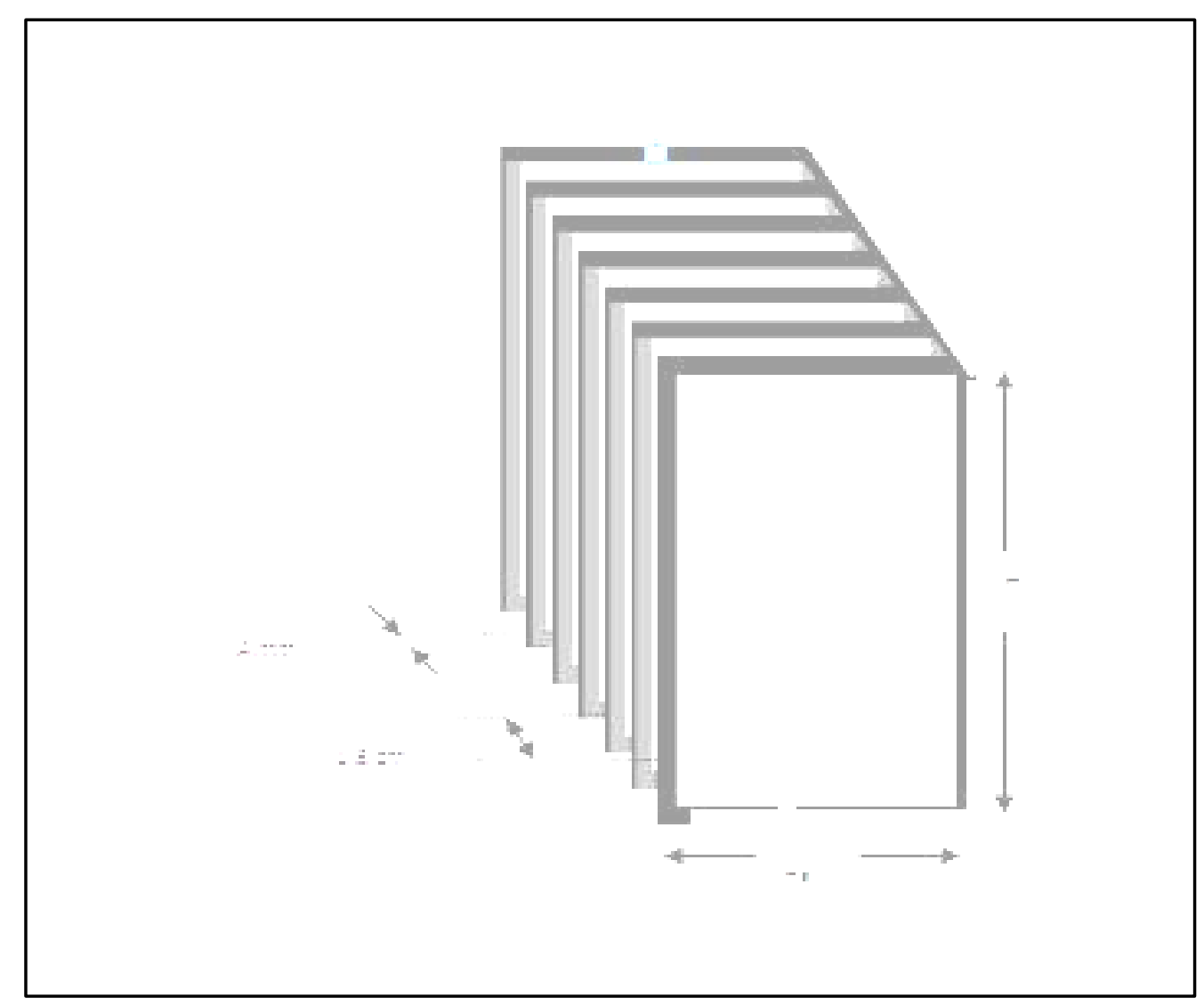

Fig.III.5: réseau d'ailette

Après avoir fixé les dimensions géométriques optimales, nous allons préciser dans cette section la simulation des échanges thermiques au niveau de la partie basse du capteur 
durant la période production de froid, plusieurs formes d'ailettes peuvent être envisagées. On commencera par le cas d'ailettes rectangulaires avec un écartement uniforme.

\subsubsection{Conditions aux limites}

L'ensemble des conditions aux limites est représenté sur la figure 6

Conditions aux limites triviales:

$\checkmark \quad$ Les faces avant et arrière sont caractérisées par une condition de semmetry.

$\checkmark \quad$ La paroi du capteur est associée à une condition de température imposée à $70^{\circ} \mathrm{C}$.

$\checkmark \quad$ La conduction dans l'ailette est prise en compte, autrement dit celle-ci est considéré comme élément "solid", de conductivité thermique $\mathrm{k}=204.4 \mathrm{Wm}^{-1} \mathrm{~K}^{-1}$, ce qui correspond à la conductivité thermique d'un matériau tel que l'aluminium.

\section{a. Conditions aux limites sur les frontières ouvertes:}

Les frontières ouvertes du domaine sont caractérisées par les conditions aux limites suivantes :

Vitesse d'entrée : cette condition permet d'imposer sur une surface une vitesse et une température du fluide entrant, la vitesse est estimée à $0.03 \mathrm{~m} / \mathrm{s}$

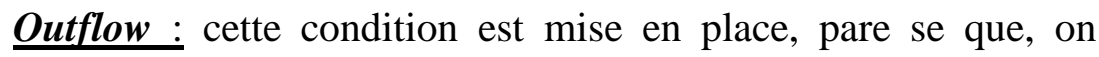
connaît pas les paramètres (vitesse et pression) du fluide sortant. 


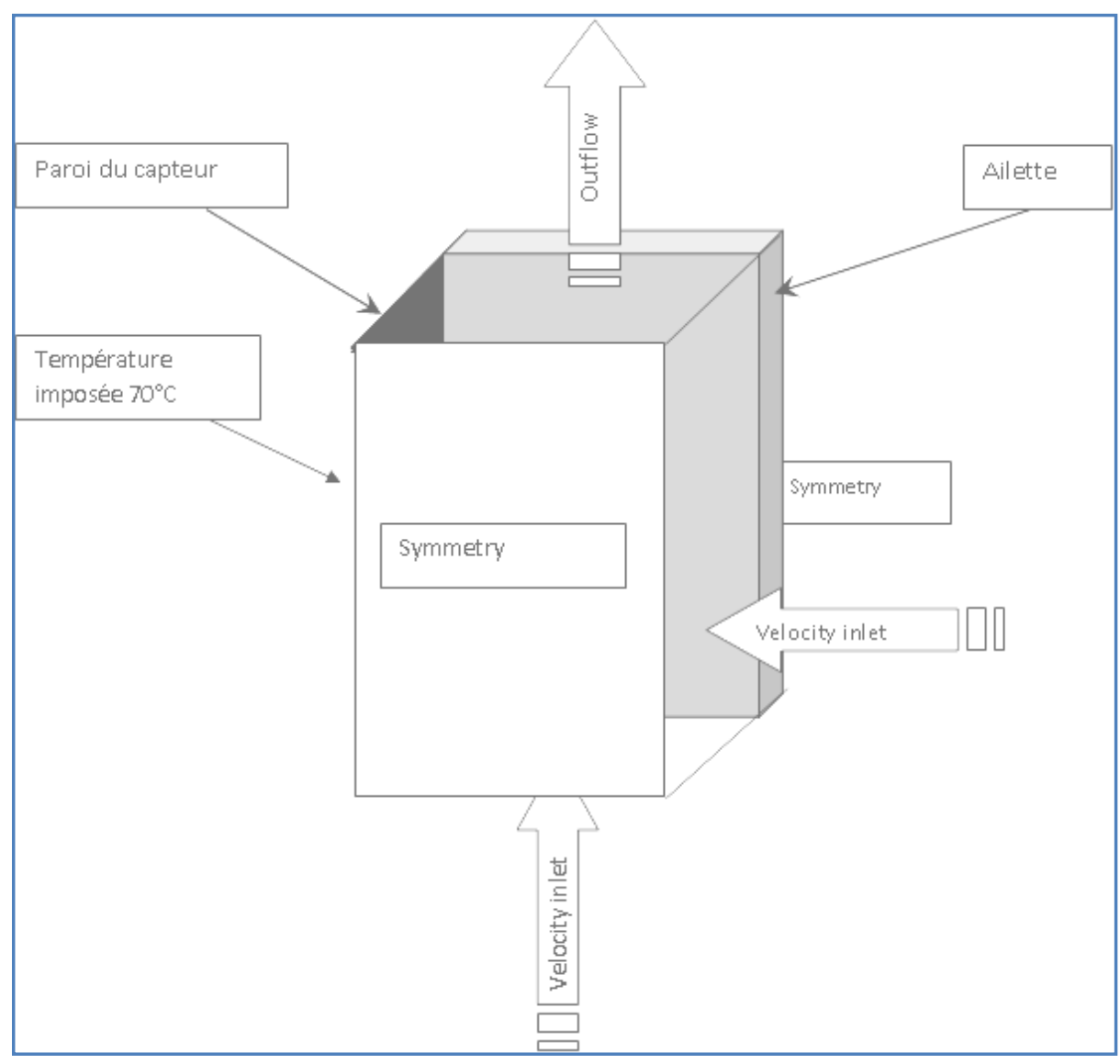

Fig.III.6 : Conditions aux limites

\subsection{Résultat}

Comme il a été déjà signalé, la problématique consiste À bien dimensionner les ailettes du capteur, pour ça, trois types d'ailettes sont proposés; rectangulaire simple, triangulaire simple et rectangulaire coupé (en haut et en bas).

La simulation est faite uniquement sur une partie du domaine, pour les trois types d'ailettes,

\subsubsection{Cas d'ailette rectangulaire simple}

Dans un premier temps, on s'est proposé d'étudier les transferts des ailettes rectangulaires, ceci nous permettre de comparer nos résultat avec ceux du LET [35]. Ces ailettes, de conception simple, peuvent être les plus efficace

Dans un deuxième temps, nous avons cherché d'autre forme d'ailettes toujours avec les mêmes conditions aux limites; 
- L'entrée bas et avant du domaine sont caractérisées par une condition de velocity inlet.

- La sortie du domaine en haut est caractérisée par une condition de outflow.

- $\quad$ L'ailette est un matériau "solid"

- Les faces avant et derrière ont une condition de semmetry.

Les résultats sont présentés sous forme de courbes, isotherme, vitesse

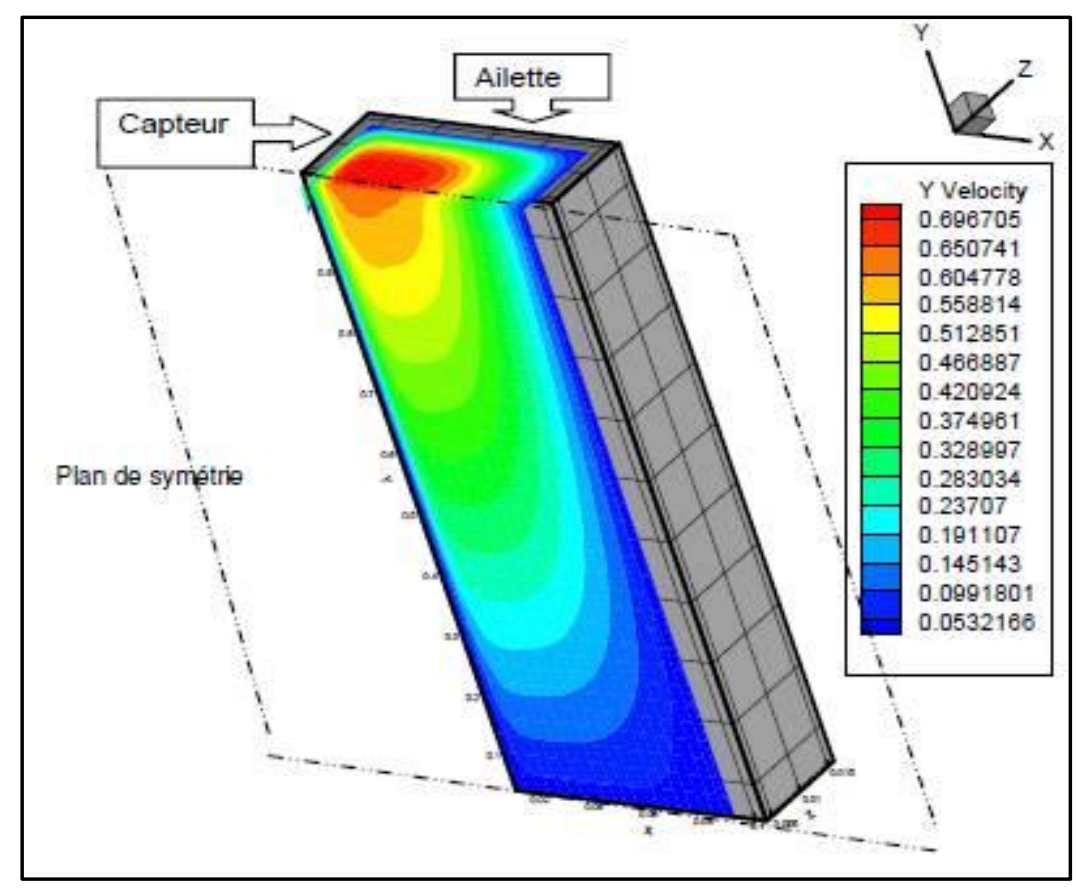

Fig.III.7 : Profil 3D de vitesse de l'air 

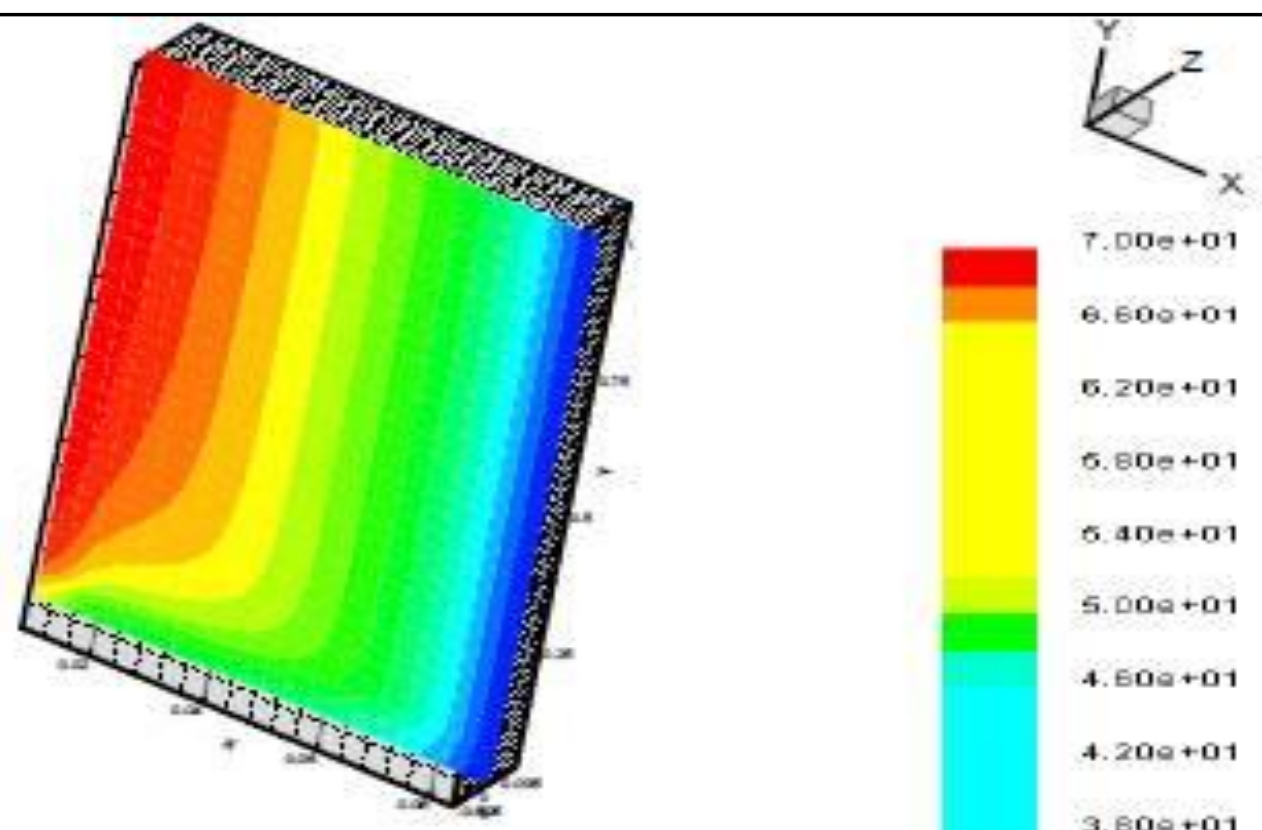

6. $600+01$

5. $200+01$

6. $80 p+01$

5. $40 e+01$

5. $000+01$

4. $600+01$

$4.200+01$

3. $800+01$

3. $40 e+01$

3. $000+01$

Fig.III.8 : profil de température sur le plan de symétrie $\left(\mathrm{T}\right.$ en $\left.{ }^{\circ} \mathrm{C}\right)$
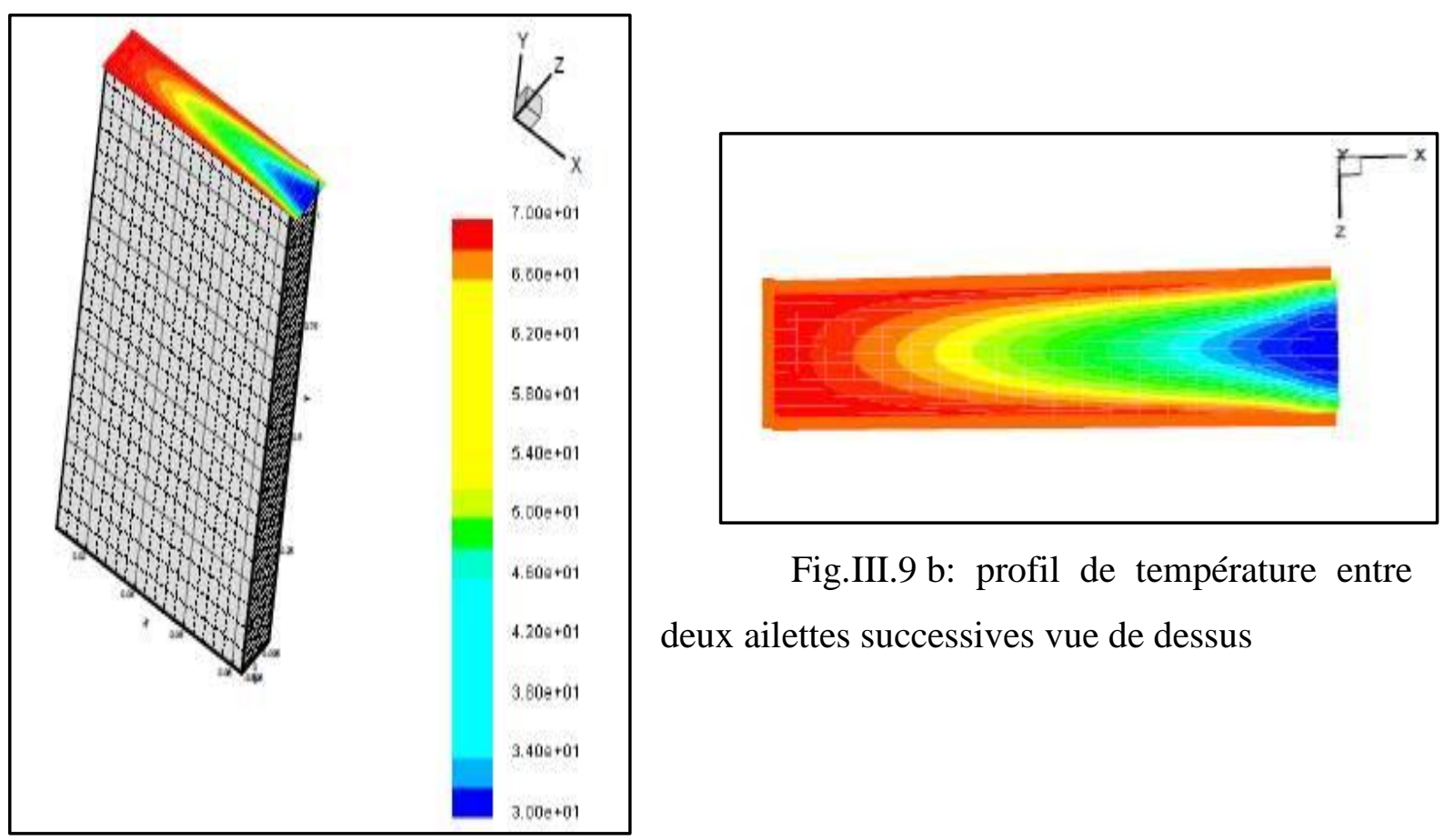

Fig.III.9 b: profil de température entre deux ailettes successives vue de dessus

Fig.III.9 a: profil de température entre deux

ailettes successives. $1 \mathrm{~m}$ en hauteur 


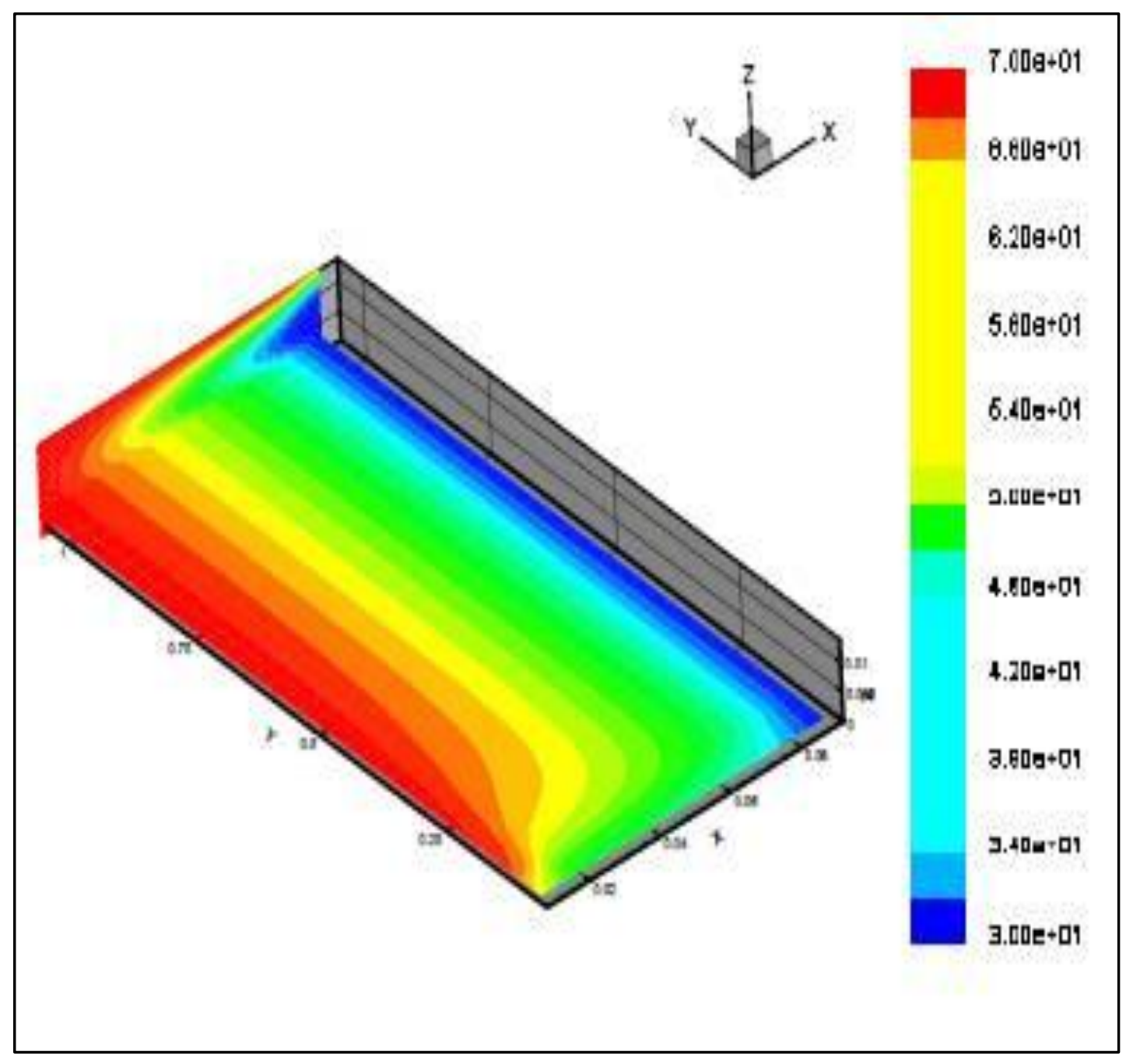

Fig.III.10: profil de température 3D

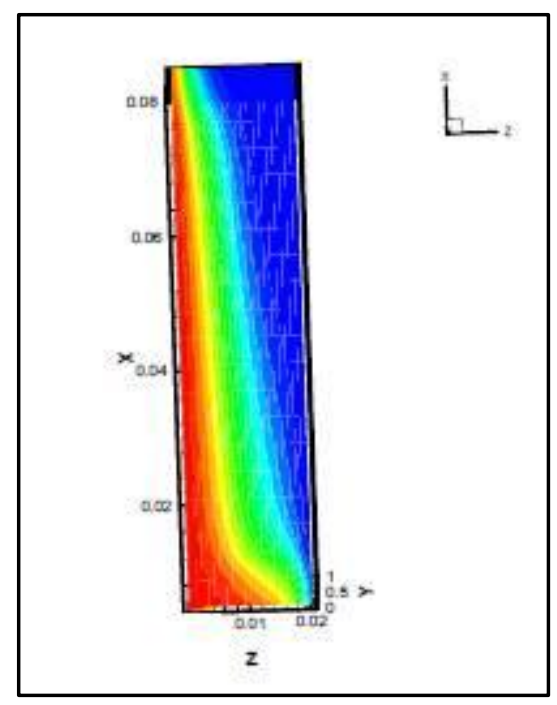

Fig.III.11: profil de température (dernière ailette) 
Sur la figure III.7 (profil de vitesse verticale plan de symétrie avant), la langueur de l'ailette prise est égale a $8 \mathrm{~cm}$ pur une épaisseur de $4 \mathrm{~mm}$, on peut observer l'évolution du profil de vitesse de l'air le long de l'ailette, à la base du capteur, la vitesse de l'air est nulle, mais au contact de l'ailette, l'air s'échauffe et une force ascensionnelle est créé permettant à l'air d'accélérer son mouvement vers le haut. Sur le sommet de l'ailette l'air atteint une vitesse maximale environ $0.7 \mathrm{~m} / \mathrm{s}$.

Rappelons que le but est de doper les échanges par convection naturelle au niveau des ailettes. Le coefficient d'échange est d'autant plus fort que la vitesse de l'air est plus importante.

Sur la figure III.7 on propose le profil des vitesses entre deux ailettes successives, on voit que la majorité des échanges sont assurés par la moitié des ailettes. L'interaction entre les ailettes y claire.

Sur les figures III.8 et III.9 on propose de voir les profils de températures sur les plans de symétries avant et le plan perpendiculaire par rapport à l'ailette. On constate clairement le développement de la couche limite de convection naturelle le long de la paroi du capteur, ce qui traduit bien l'effet de refroidissement du capteur.

Le choix de ce type d'ailette demeure encourageant vu leurs simplicités technologiques, et les résultats trouvé.

\subsubsection{Cas d'ailette triangulaire simple}

Afin d'enrichir d'avantage notre étude, et de la rendre, finalement, plus complète, nous nous sommes proposé de changer la forme des ailettes. A priori, ce changement aura une influence sur les échanges thermiques par convection [33].

Notre choix s'est porté sur des ailettes de forme triangulaire simple. Nous nous sommes forcé à garder les mêmes données géométriques (même volume d'ailettes) pour pouvoir, par la suite, comparer les résultats avec ceux des ailettes rectangulaires. Les conditions aux limites seront les mêmes que ceux appliquées pour le cas précédent.

La figure III.11 représente la forme et les dimensions choisies pour notre calcul. 


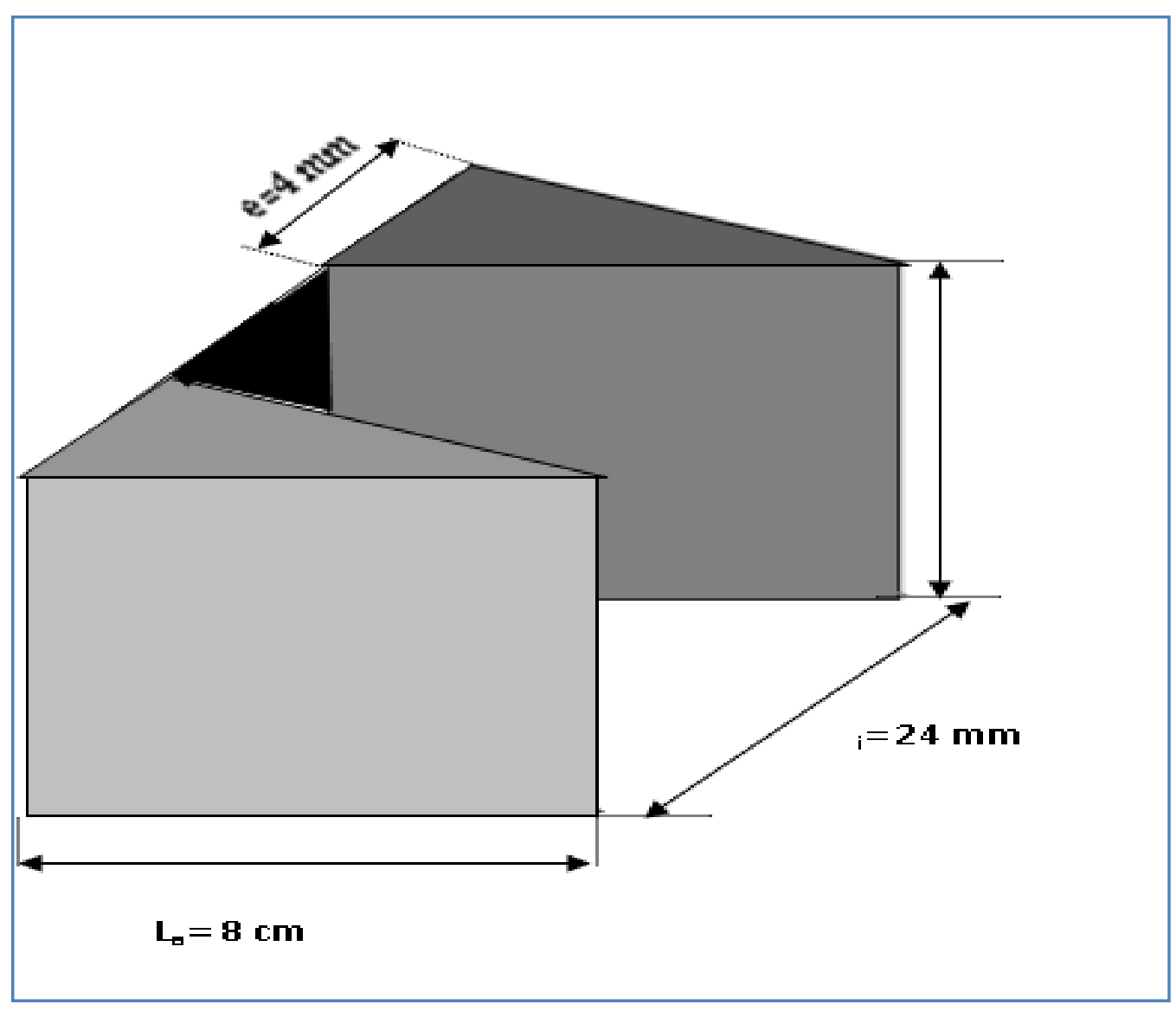

Fig.III.12: Ailettes triangulaires

Nos avons eu beaucoup de problèmes d'instabilité numériques à cause de la forme particulières étudiées, néanmoins, nous avons réussi à trouver quelques résultats qui sont présentés sous forme d'isotherme et de champ de vitesses. 


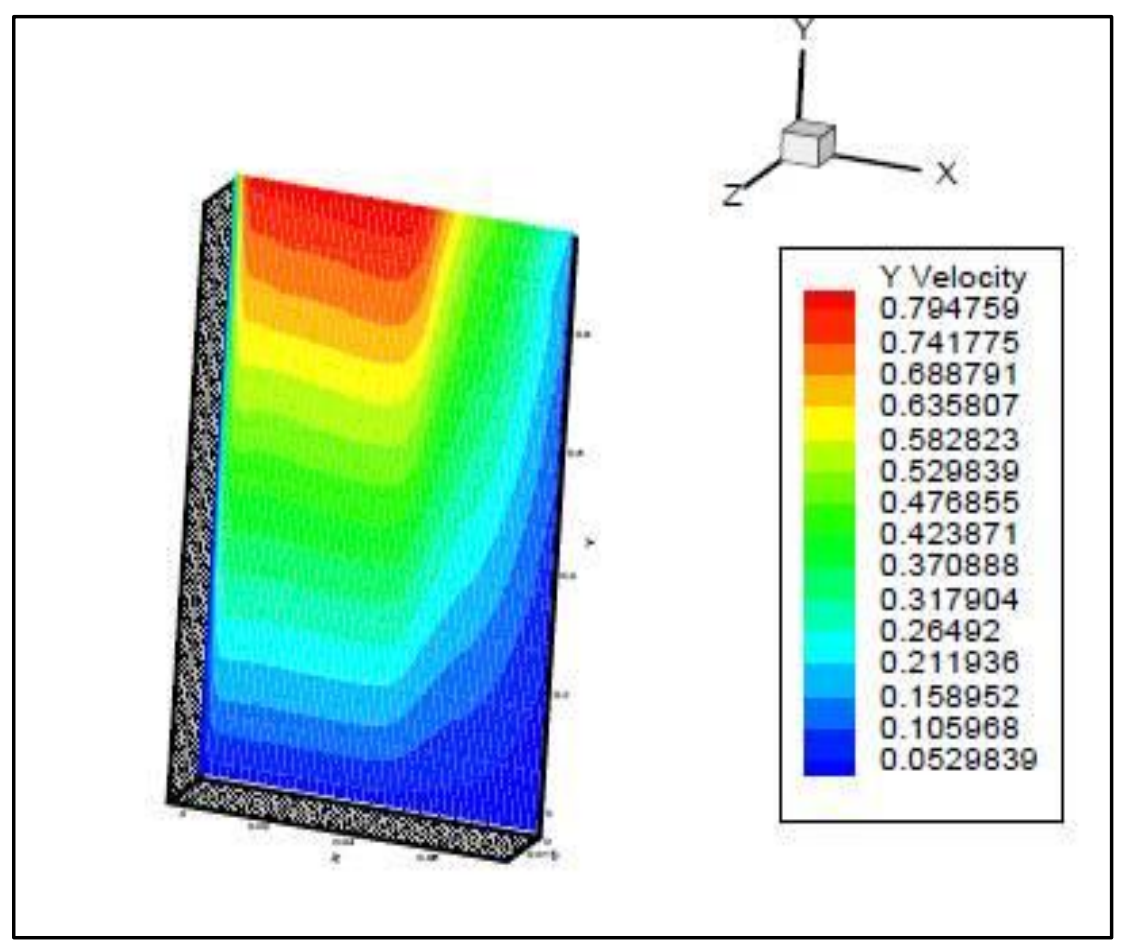

Fig.III.13: profil de vitesse de l'air plan de symétrie avant

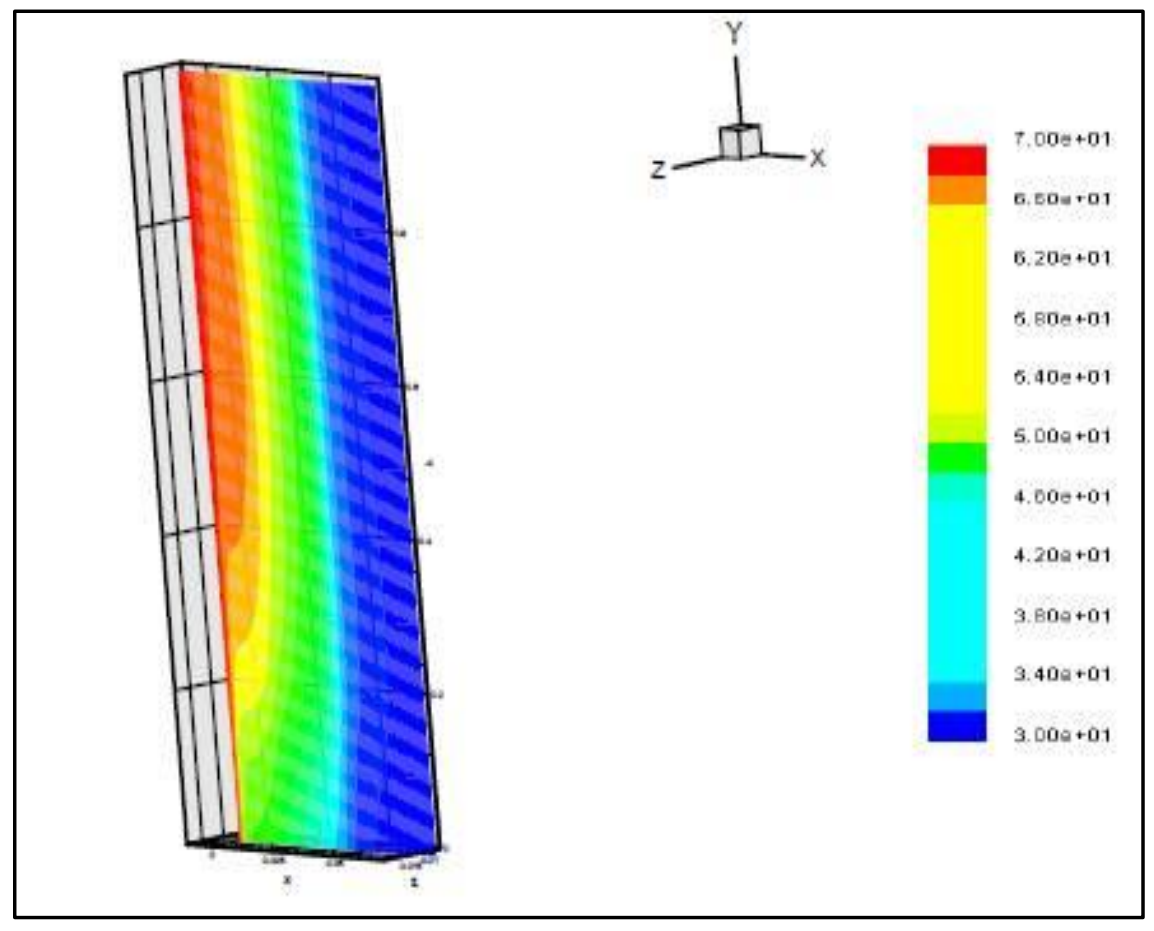

Fig.III.14: profil de température plan de symétrie avant 


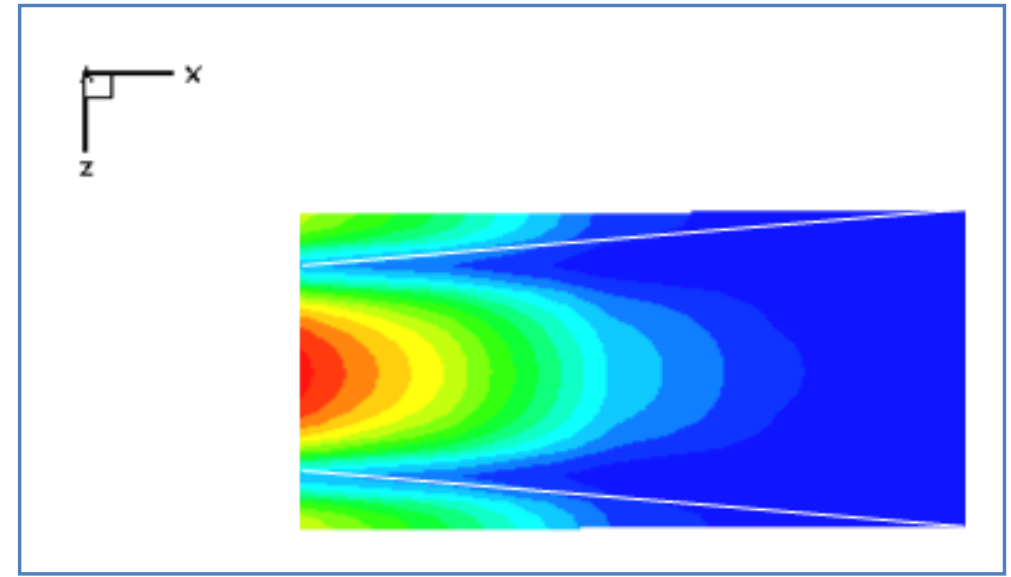

Fig.III.15: profil de température entre deux ailettes successive.

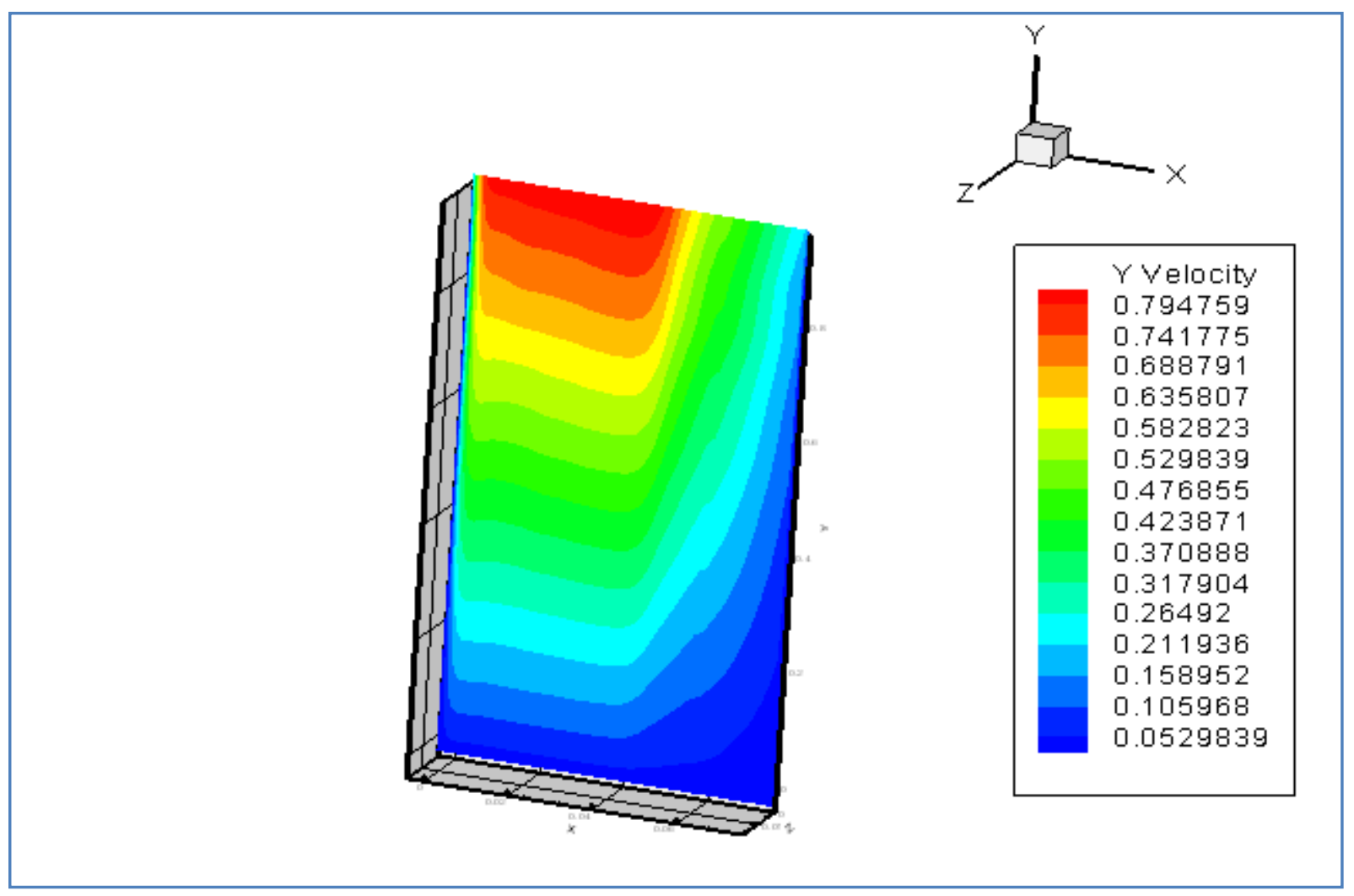

Fig.III.16: profil de vitesse de 1'air plan de symétrie. 


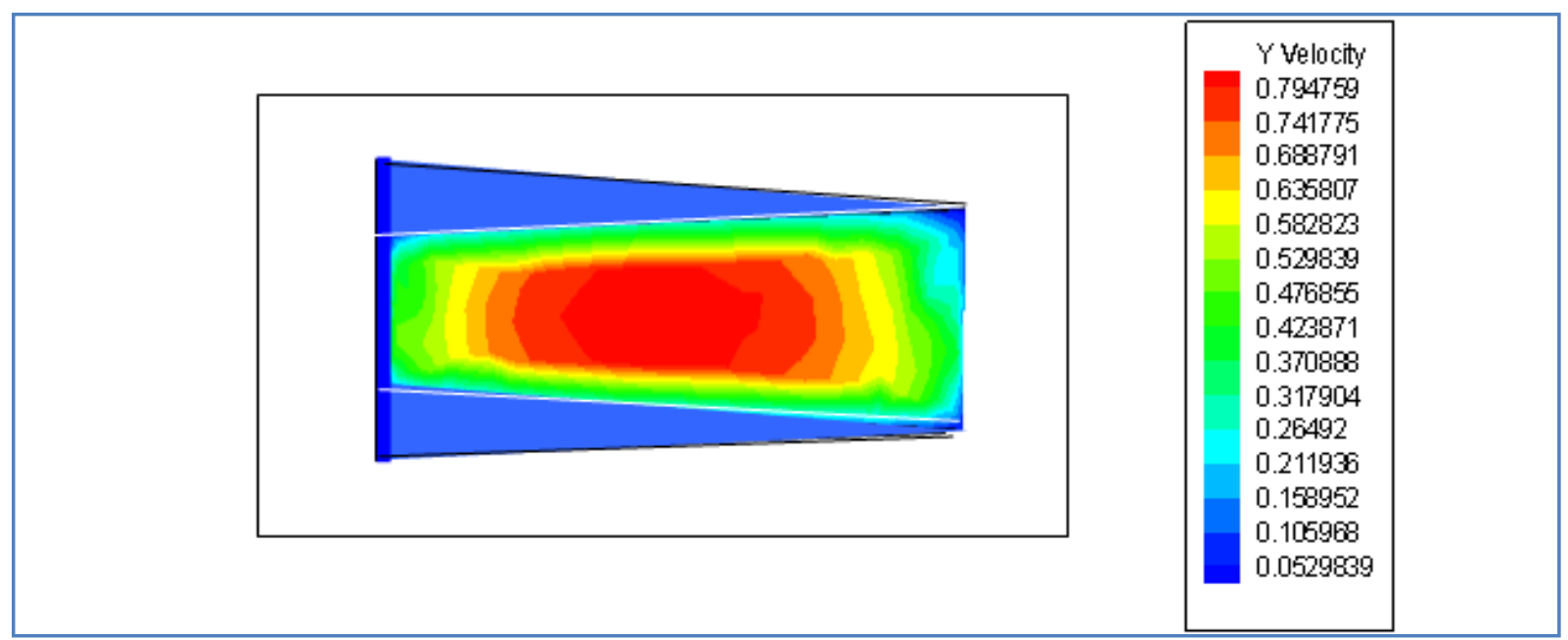

Fig.III.17: profil de vitesse de l'air entre deux ailettes successives plan ' $\mathrm{y}=1 \mathrm{~m} \perp$

à l'ailette'.

On voit clairement l'ailette est plus "chaude" que l'ailette rectangulaire ce qui entraîne une efficacité meilleure. On peut dire aussi que près des deux tiers de l'ailette efficacement le transfert de chaleur vers l'air ambiant. Sur cette figure, et comparativement avec l'étude précédente on voit clairement que les isothermes sont plus étendues et donc la convection est plus manquée.

Pour les champs de vitesses on peut dire a travers les figures présentées que les résultats trouvées ne sont pas certainement loin de la réalité, des probèmes d'instabilités numérique sont à l'origine des problèmes à l'apprche de la paroi du capteur et vers le bout des ailettes.

L'essentiel, on peut généralement faire remarque que celles des isothermes, la convection est plus accordée par rapport aux ailettes rectangulaires.

En conclusion, on peut dire que l'implantation d'ailettes triangulaire est meilleure (théoriquement) que celles d'ailettes rectangulaires. Cette solution reste un peu plus compliquée technologiquement.

Nous avons essayé de simuler les échanges avec des ailettes rectangulaires coupé des cotés haut et bas, des problèmes de temps alloué et de puissance du matériel de calcul (ordinateur) nous ont obligé à se limiter à ces deux cas de forme seulement. 
Nous espérons qu'une étude complémentaire sera lancée pour enrichir celle-ci, la réalisation de ce capteur

\subsection{Sensibilité au maillage :}

Afin de confirmer les résultats trouvés, nous avons effectué un changement de paramètre de maillage. L'étude de sensibilité au a été fait par le jeu des paramètres suivants :

$$
\begin{aligned}
-\vec{g} & =g \vec{y} \\
i & =16 \mathrm{~cm} \\
e & =4 \mathrm{~mm} \\
L_{a} & =8 \mathrm{~cm}
\end{aligned}
$$

La figure 8 représente l'influence de l'affinage du maillage sur le profil de température et la composante verticale de la vitesse dans le plan de symétrie avant à mi-hauteur du capteur.

La courbe noire est celle du maillage de base alors que la violet est celle obtenue avec le maillage fin (la moitié du pas), et enfin la jaune est celle du maillage large (le double du pas). On remarque le développement de la couche de limite développé le long de la paroi du capteur. On voit clairement que les résultats trouvés ne présentent qu'une légère sensibilité au maillage.
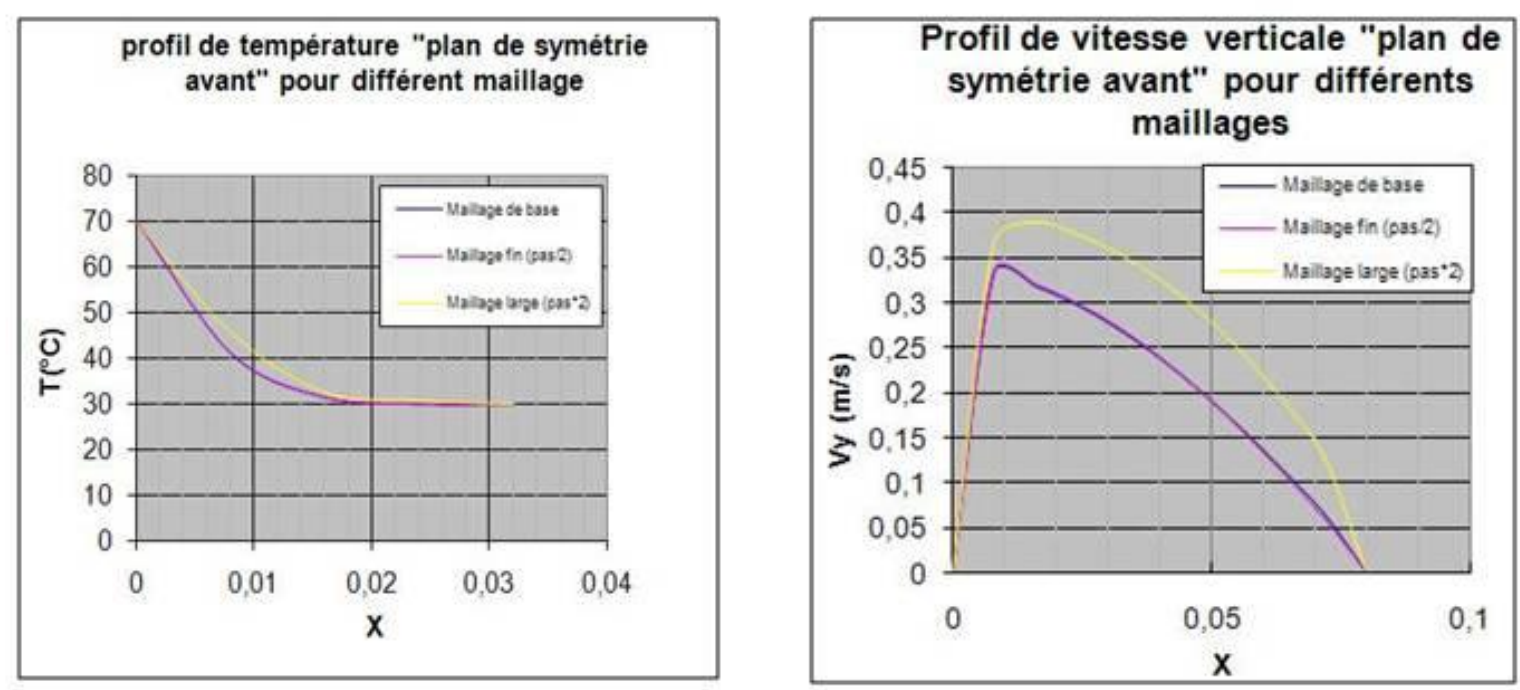

Fig.III.18 : sensibilité au maillage 
Chapitre IV

\section{Description de l'installation expérimentale et analyse des résultats}

\subsection{Introduction}

Au niveau mondial plusieurs prototype ont été fabriqués et testés. Alors on Algérie et à notre connaissance aucun dispositif expérimental n'a était réalisé.

Plusieurs prototype utilisant les couple charbon-actif -méthanol [57, 58, 59, 60, 61, 62] et zéolithe-eau $[63,64,65,66,67,68]$ ont été réalisé et testé dans le monde, alors le couple silicagel-eau n'a pas eu sa chance.

En 1986 SKODA, M et Suzuki ont testé et expérimenté une machine frigorifique solaire à adsorption utilisant le couple gel de silice-eau, le capteur utilisé est de $0.25 \mathrm{~m}^{2}$, d'épaisseur de $5 \mathrm{~cm}$ contenant un kilogramme de gel de silice. D'après ces chercheurs le COP de l'installation avait atteint 0.2 et 0.4 pour une surface de capteur égal à $0.4 \mathrm{~m}^{2}$ [69].

En 2000 ; l'équipe de Philippe Dind a fabriqué et testé un congélateur solaire à adsorption utilisant le couple gel de silice - eau, la surface du capteur est de $2 \mathrm{~m}^{2}$, d'après cette équipe le COP a atteint une valeur de 0.19 sous un ensoleillement artificielle [70]. 


\subsection{Description de l'installation expérimentale}

Le prototype de la machine frigorifique solaire à adsorption que nous avons fabriqué dans le département de génie mécanique à l'université Mohamed khider de Biskra et qui sera testé en premier temps à Oum el Bouaghui utilise le couple gel de silice - eau, on a opté pour ce choix, vu la disponibilité et le cout du gel de silice dans le marché algérien.

Il se compose d'un capteur solaire, d'un condenseur et d'une chambre froide contenants l'évaporateur.

\subsubsection{Le Capteur :}

Le capteur est un parallélépipède de côté : $10 \mathrm{~mm}$ X $85 \mathrm{~mm}$ X $85 \mathrm{~mm}$. Il est construit par des tôles d'aluminium d'épaisseur $1.5 \mathrm{~mm}$, qu'on a utilisé pour la première fois dans le domaine de froid solaire, on tenant compte du prix très élevé des tôle de cuivre malgré son efficacité, rempli d'un lit de gel de silice, le tous à l'intérieur d'un boitier en bois, une grande importance est donnée à l'étanchéité par l'utilisation de la silicone afin de limité toute perte.

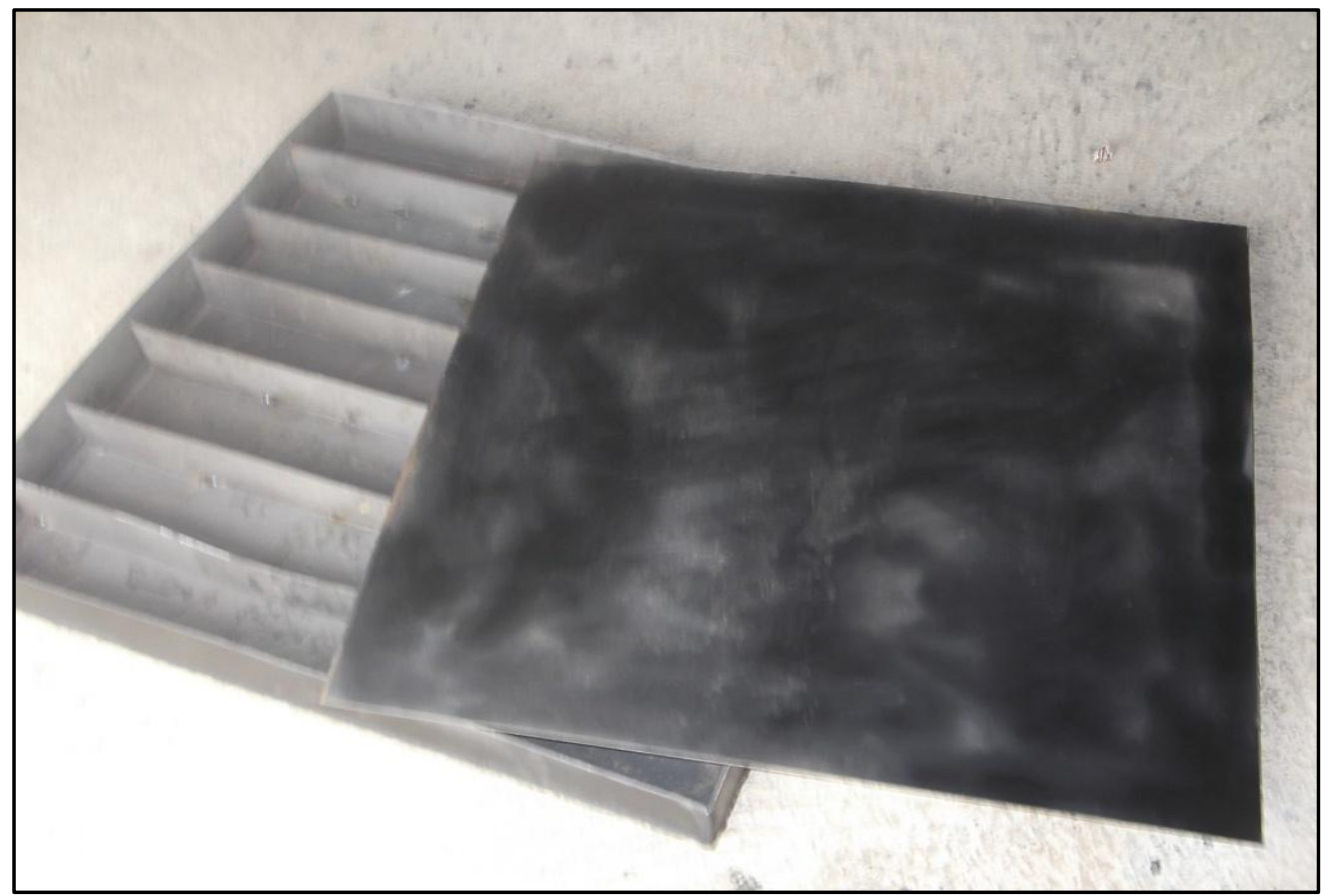

Fig. IV.1 : Vue du capteur avant l'assemblage 


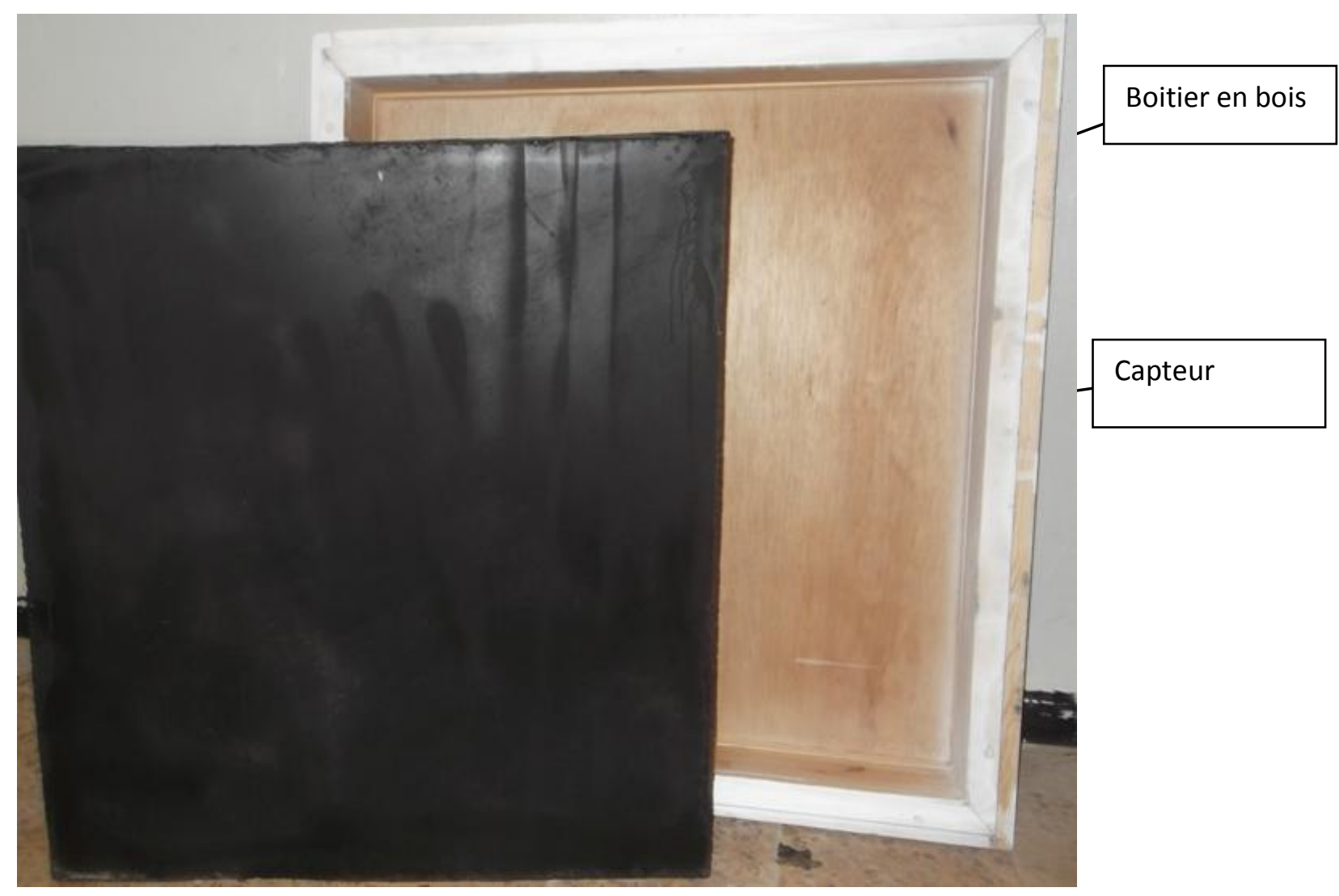

Fig. IV.2 Capteur avec le boité en bois

\subsubsection{Le Condenseur :}

Est un échangeur de chaleur qui sert assurer la condensation. Le condenseur à air utilisé dans l'installation expérimentale est disponible dans le marché algérien avec un prix très raisonnable d'environ $4500 \mathrm{DA}$, on a opté pour ce choix pour minimiser le cout ainsi le temps de fabrication du prototype expérimentale. 


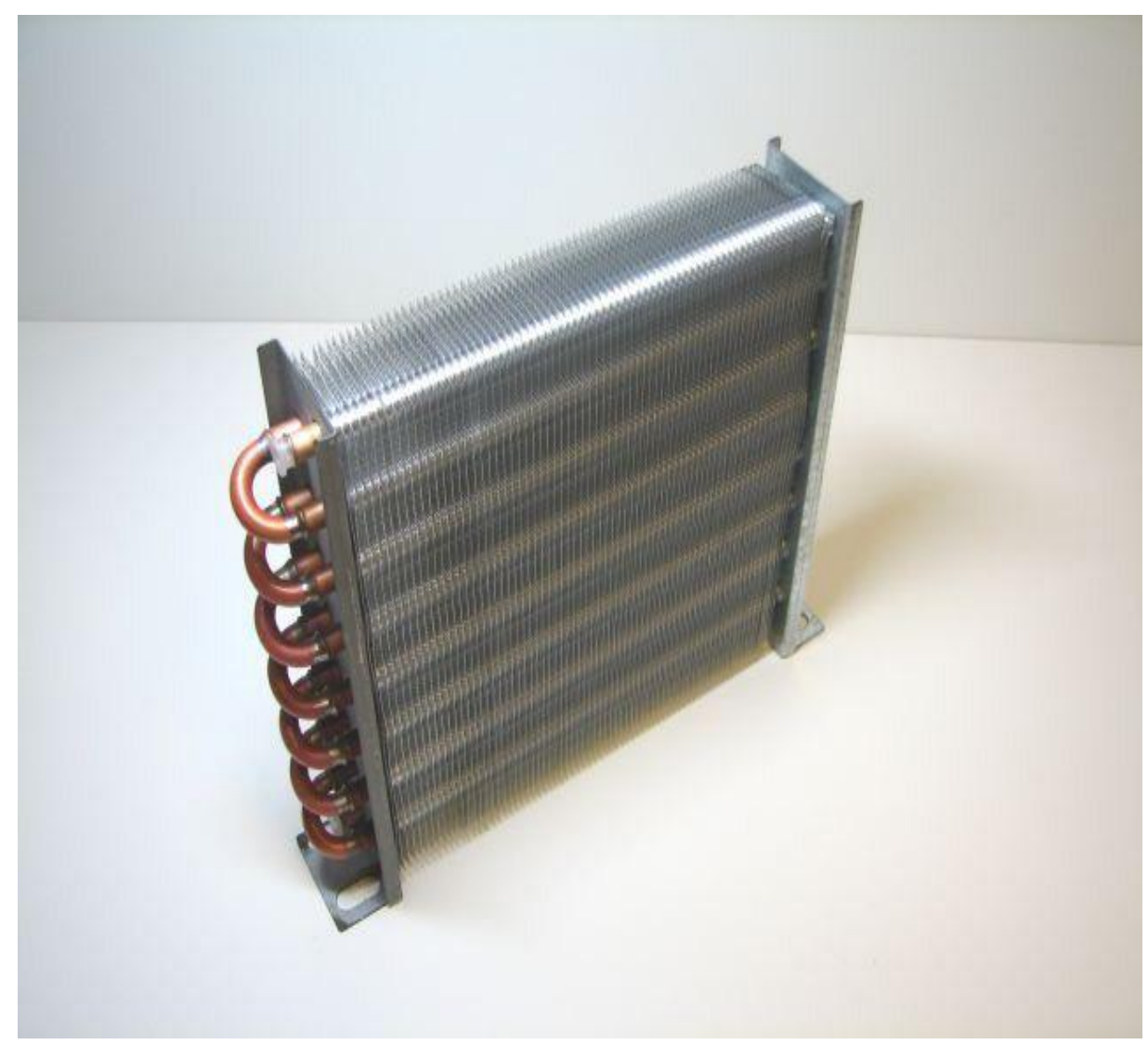

Fig. IV.3 : Condenseur à air

\subsubsection{La chambre froide :}

C'est un parallélépipède de côté $45 \mathrm{~cm}$ X $45 \mathrm{cmx} 45 \mathrm{~cm}$, construit par des Panneaux Sandwich monobloc, avec deux tôles métalliques isolés par une couche de mousse polyuréthane, de portance élevée et d'une grande rigidité. Ces panneaux sont disponible dans le marché algérien, fabriqué par une entreprise situé à M'sila (Algérie).

Il est noté ici qu'une étude de plusieurs chambre de froides construises par plusieurs matériaux a donné l'avenage à ces panneaux, afin de minimiser les pertes thermique entre l'intérieur du chambre et l'ambiant.

A l'intérieur de la chambre froide on trouve l'évaporateur, c'est un petit serpentin en cuivre de diamètre $12 \mathrm{~mm}$ et d'épaisseur de $1.6 \mathrm{~mm}$. 


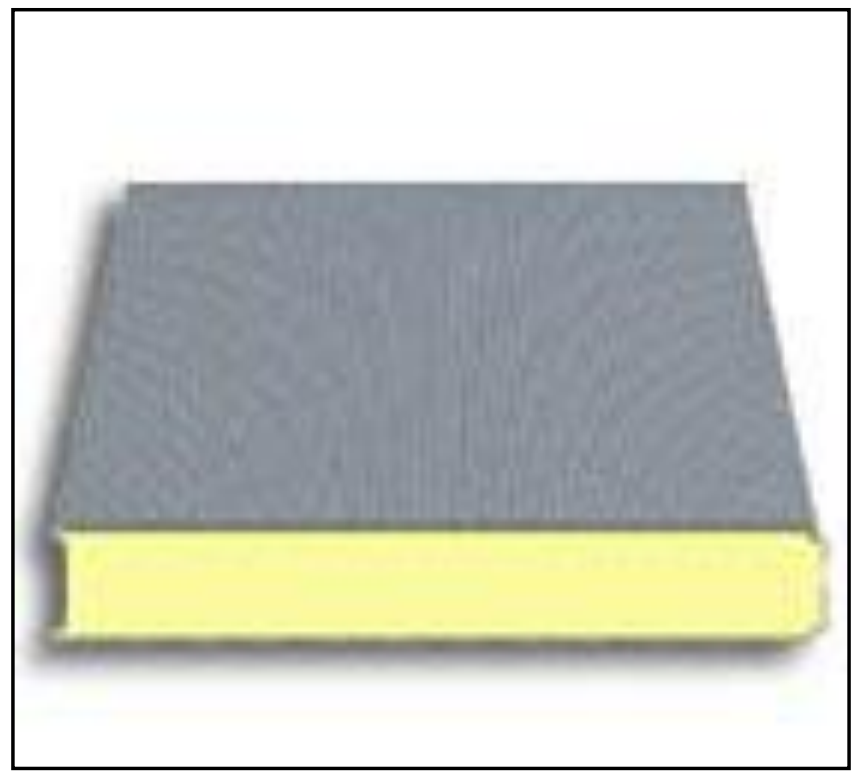

Fig. IV.4 : panneau sandwich

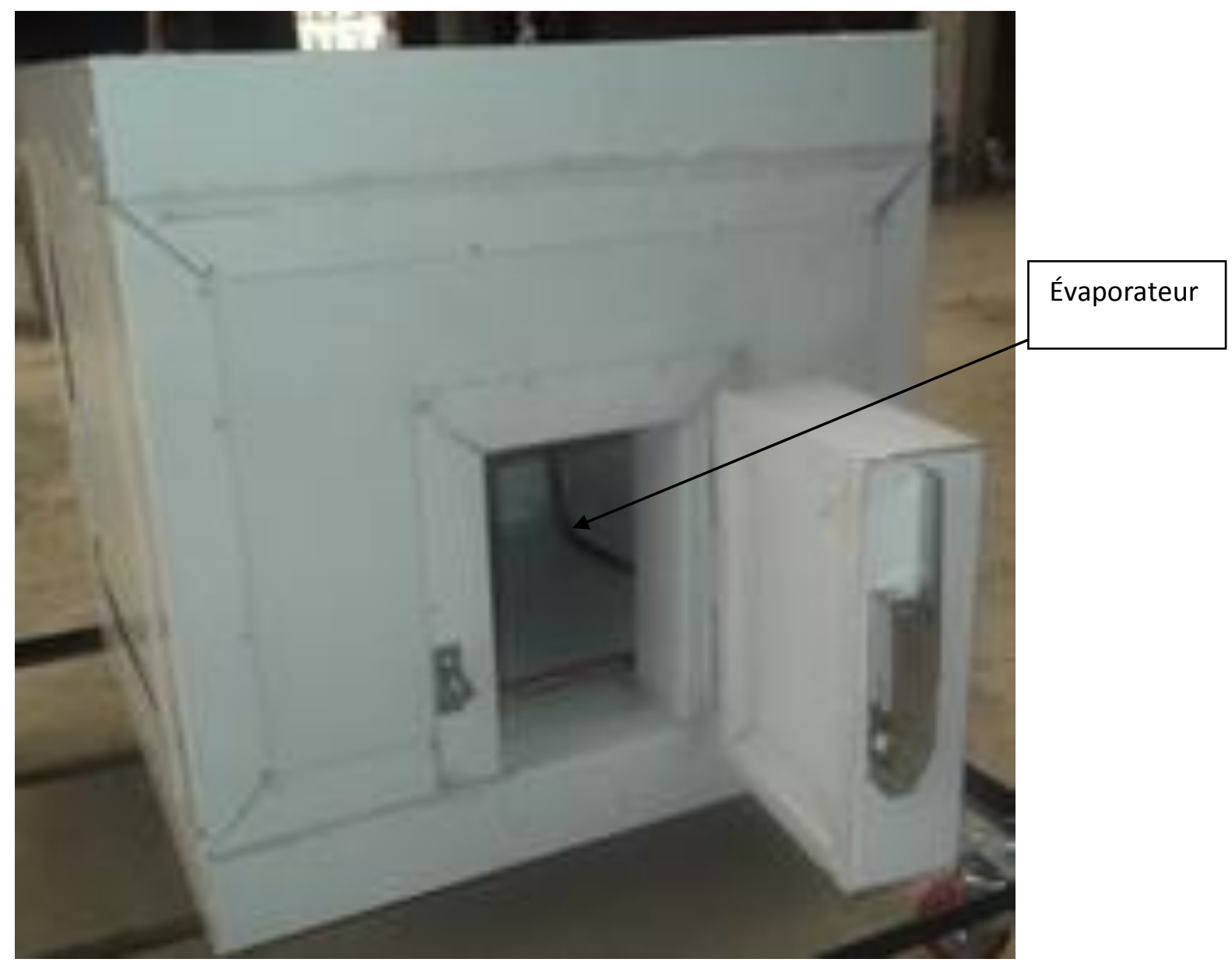

Fig. IV.5 : chambre froide contenant l'évaporateur 


\begin{tabular}{|c|c|c|c|}
\hline Epaisseur $(\mathbf{m m})$ & Poids $\left(\mathbf{k g} / \mathbf{m}^{\mathbf{2}}\right)$ & $\begin{array}{l}\text { Résistance à la } \\
\text { conductibilité } \\
(\mathbf{1} / \sqrt{\mathbf{\Omega}})\end{array}$ & $\begin{array}{l}\text { Coefficient de } \\
\text { Transmission Thermique } \\
\mathbf{k} \mathbf{~ e n}\left(\mathbf{W} / \mathbf{m}^{\mathbf{2}} \mathbf{. K}\right)\end{array}$ \\
\hline 70 & 11.38 & 2.09 & 0.41 \\
\hline 75 & 11.62 & 2.32 & 0.37 \\
\hline 80 & 11.86 & 2.54 & 0.33 \\
\hline 100 & 12.84 & 3.49 & 0.24 \\
\hline
\end{tabular}

Tableau : 4.1 caractéristique technique du panneau sandwich

4.3.5 Accessoire de liaison: les différents constituants du prototype expérimental sont reliés par des accessoires de liaison à savoir :

\section{- Tuyau flexible :}

Sont des éléments de liaison entre le capteur, le condenseur à air et l'évaporateur.

\section{- Vannes :}

Des vannes sont installées pour assurer l'intervention manuelle à chaque cycle.

\subsection{Résultat expérimentaux}

Le fonctionnement de notre machine frigorifique solaire à adsorption se résume en deux cycles :

\subsubsection{Cycle diurne :}

Le soleil chauffe l'adsorbant situé en dans le capteur se qui favorise la réaction endothermique de désorption, dans ce cas la pression augmente et la vapeur d'eau produite se condense.

4.4.2 Cycle nocturne : Dans ce cycle et a cause de l'ouverture de la plaque arrière du capteur, l'adsorbant se refroidi et perd de la pression, l'énergie extraite de l'évaporateur favorise cette réaction exothermique et donc la production du froid. 


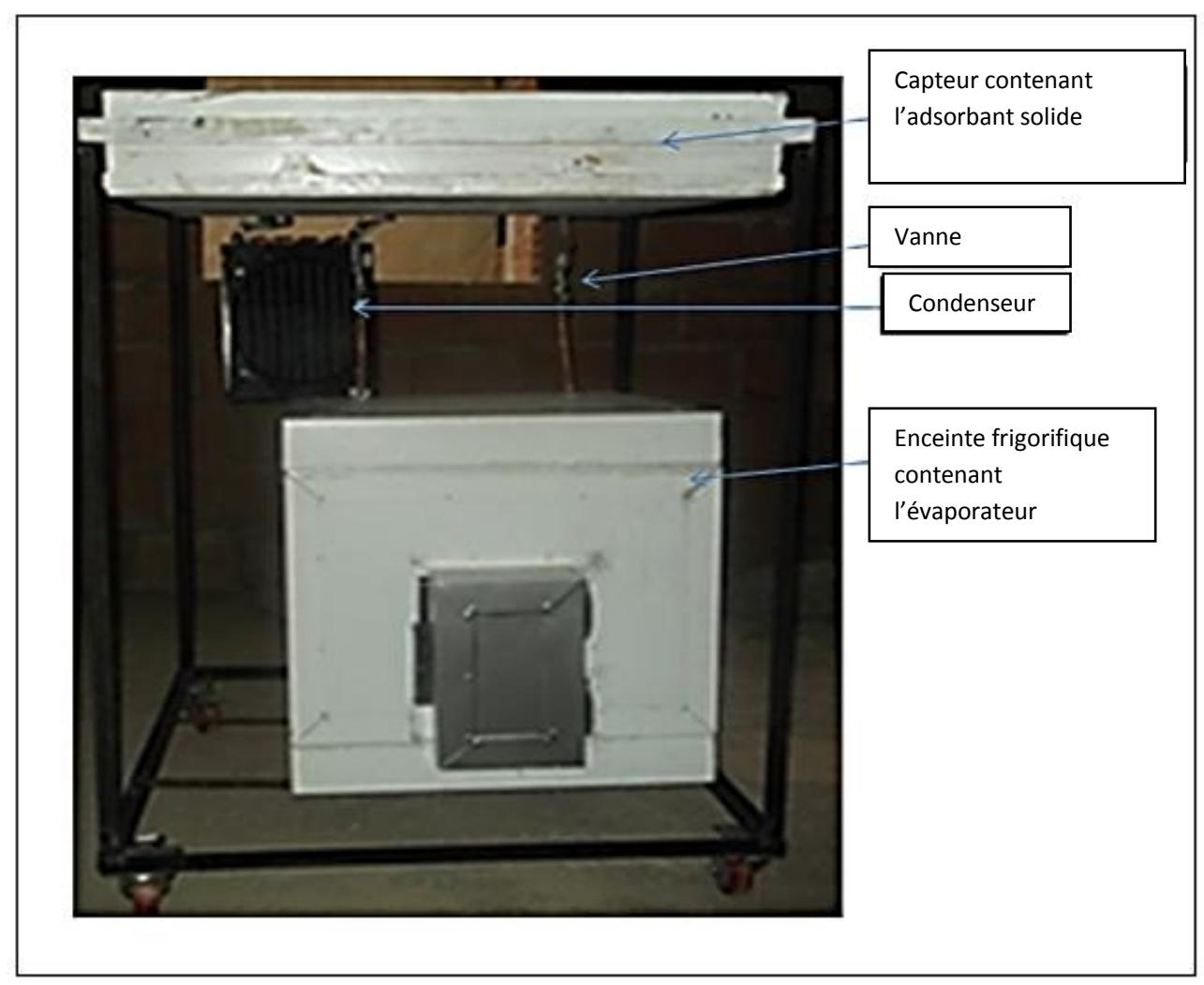

Fig. IV.6: photo de l'installation expérimentale

\subsection{Analyse des résultats :}

Afin de permettre la prise et l'analyse des résultats, des thermomètres digitaux sont fixé sur le capteur, le condenseur et l'enceinte frigorifique contenante l'évaporateur, pour mesurer les différentes températures, la limite de nos moyens nous a obligés de faire un grand effort pour enregistrer quelque résultats. Parmi les résultats obtenus, ceux des journées 01

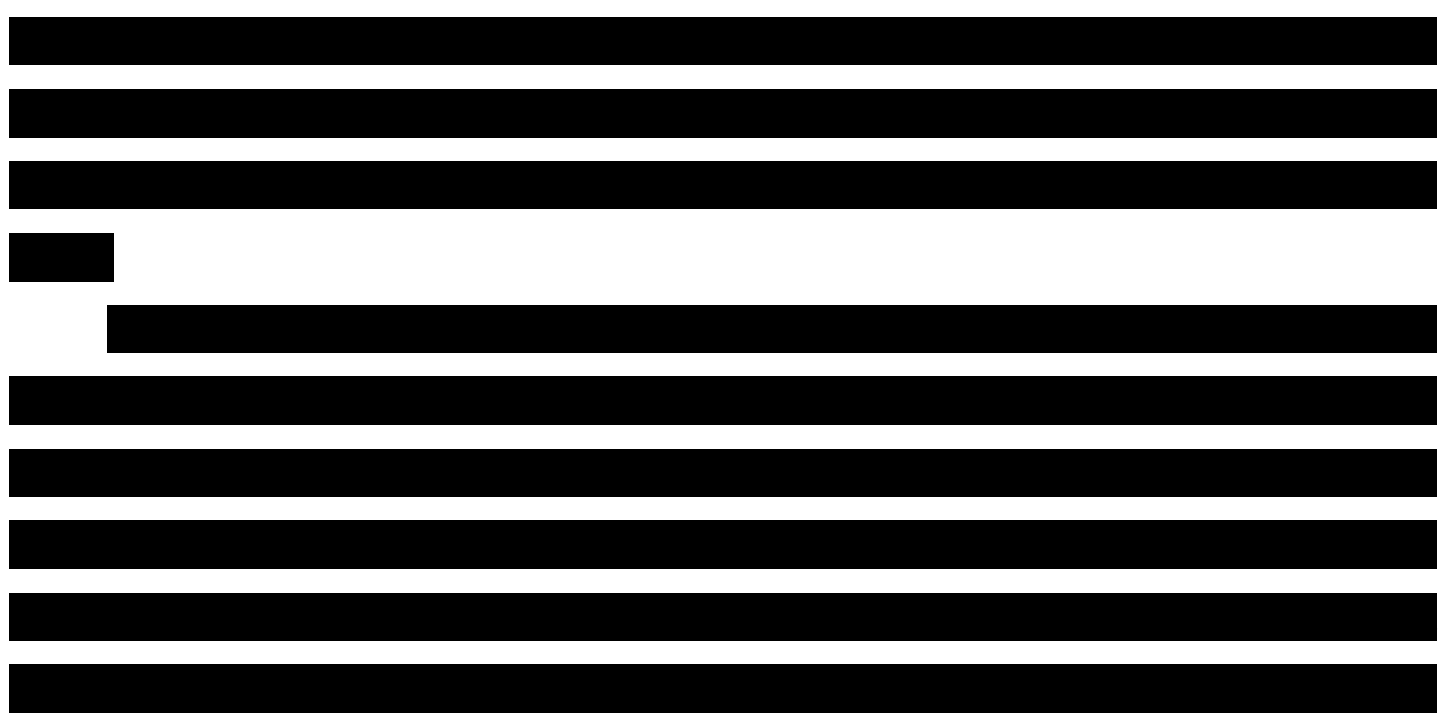




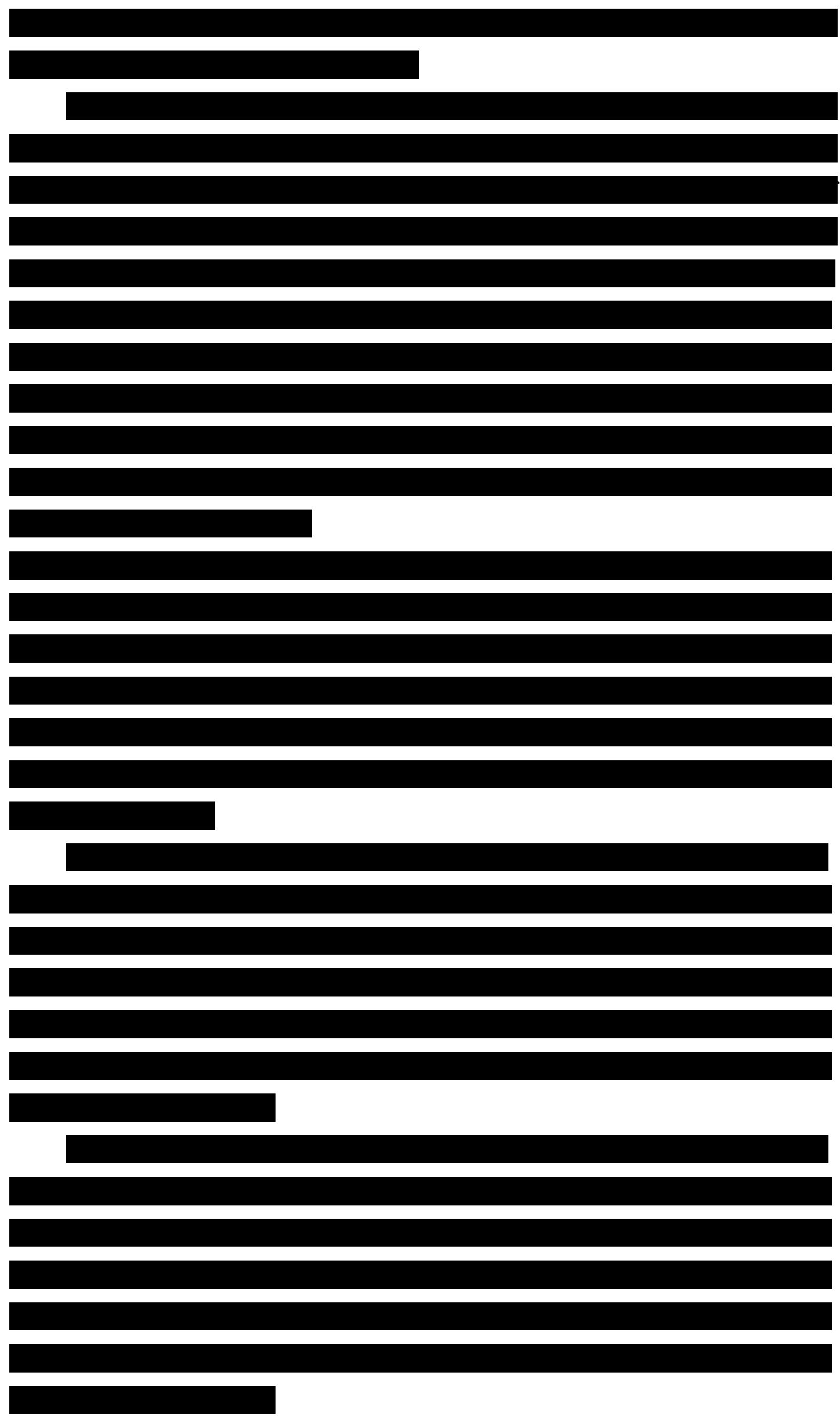




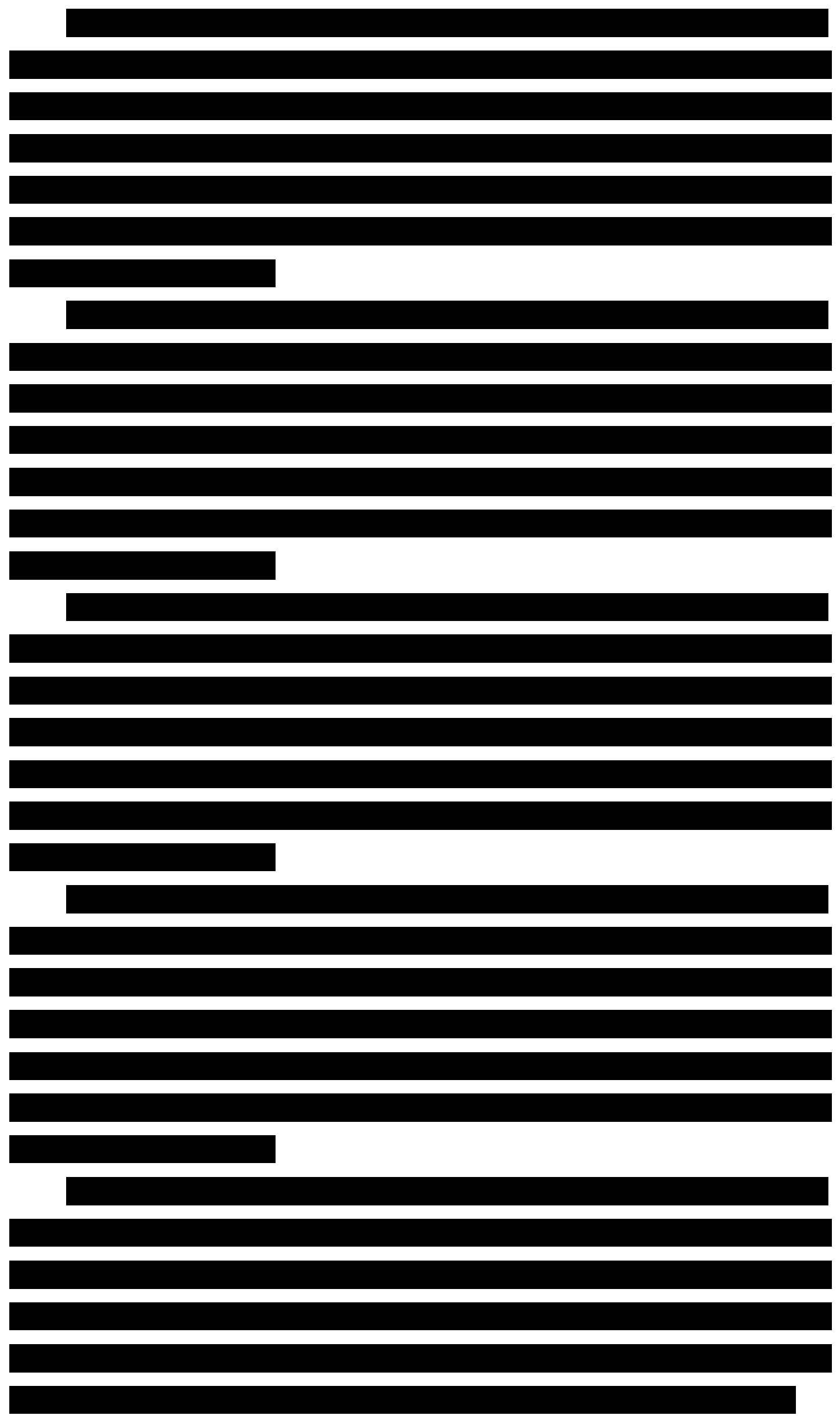




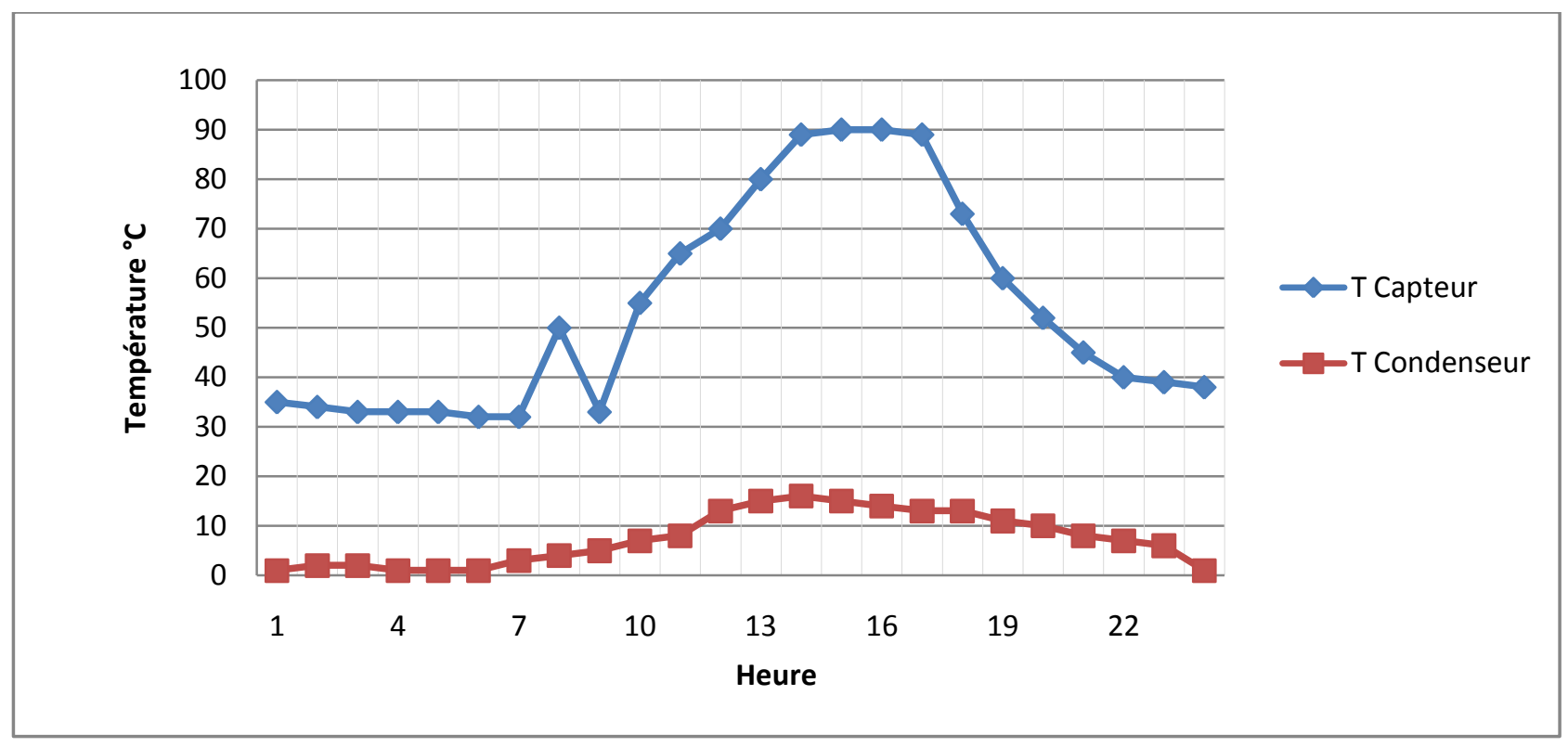

Fig. IV.7: Températures enregistrées journée du 01 mars 2015

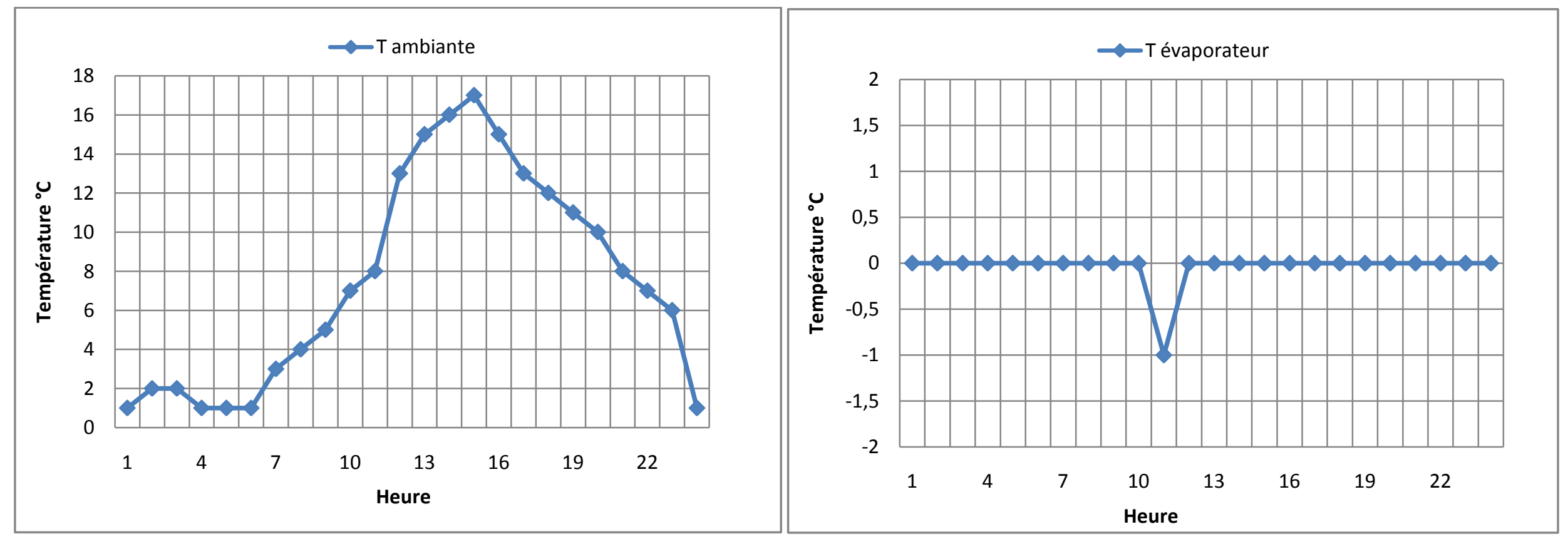




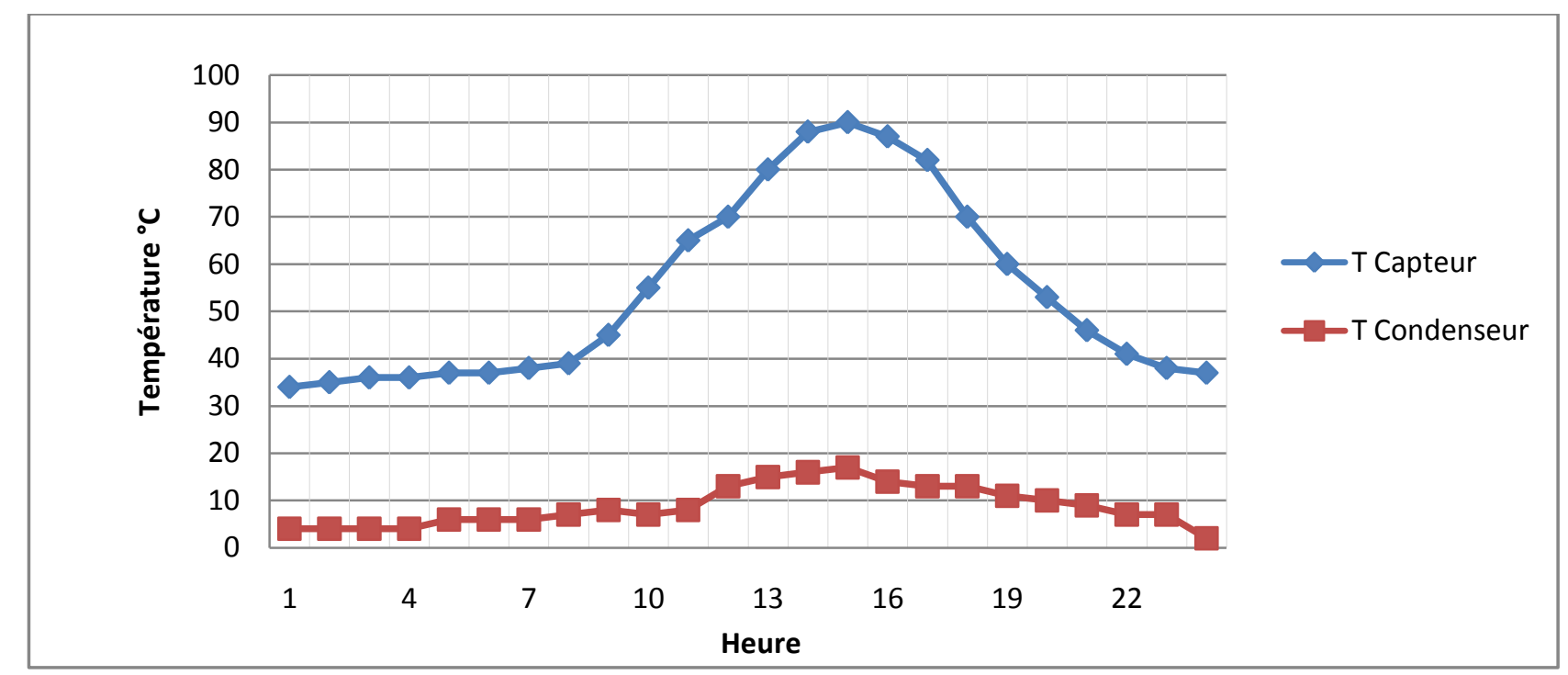

Fig. IV.8 : Températures enregistrées journée du 24 mars 2015

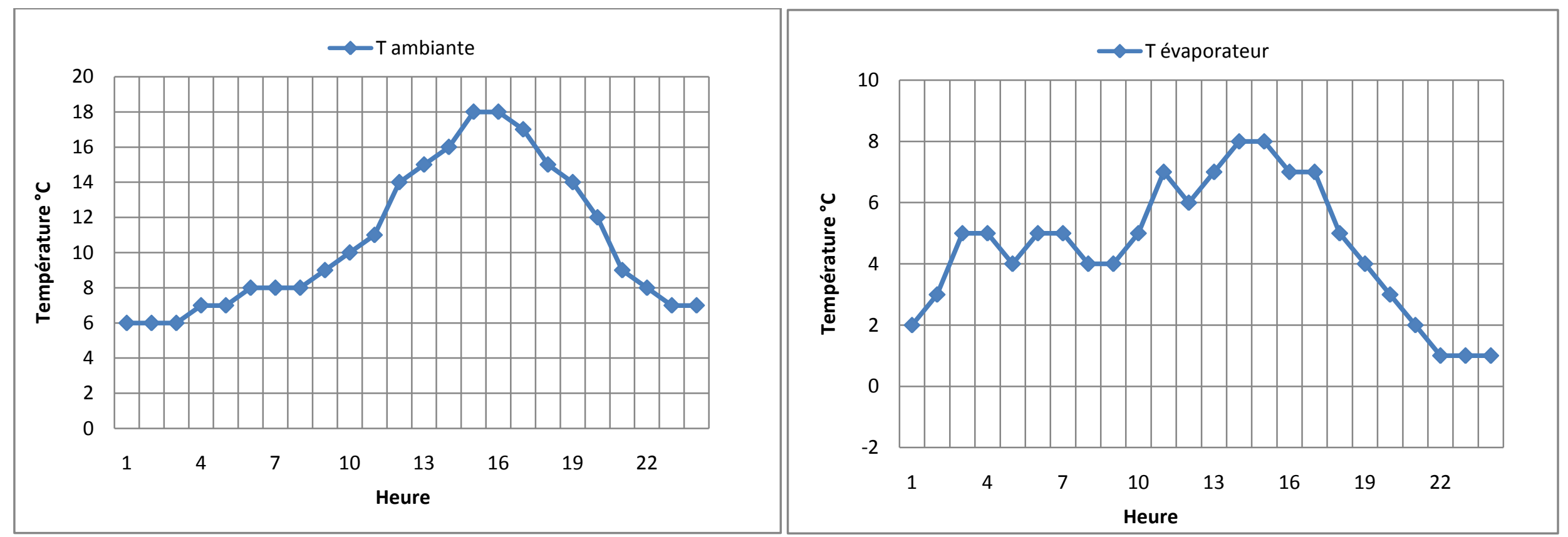




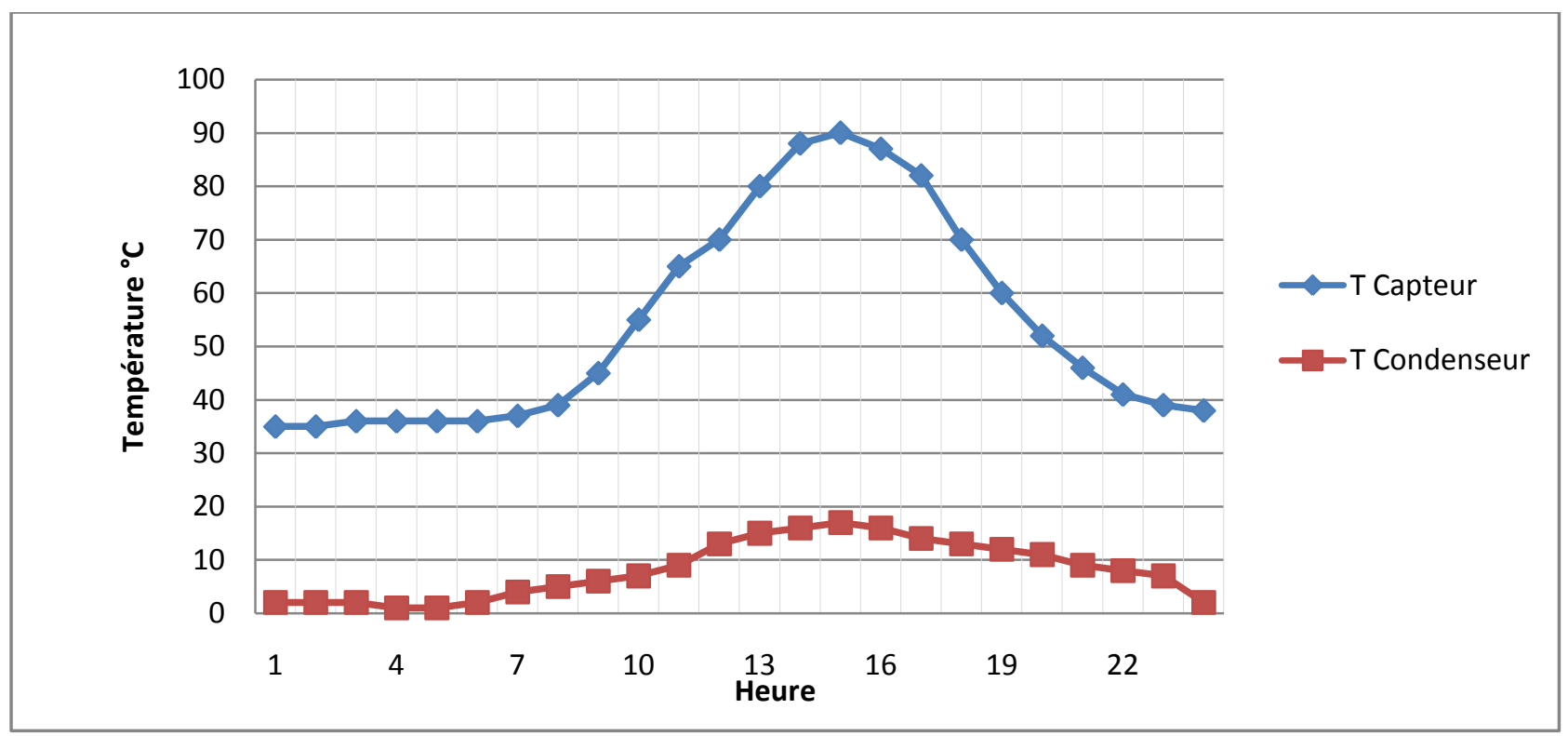

Fig. IV.9: Températures enregistrées journée du 27 mars 2015

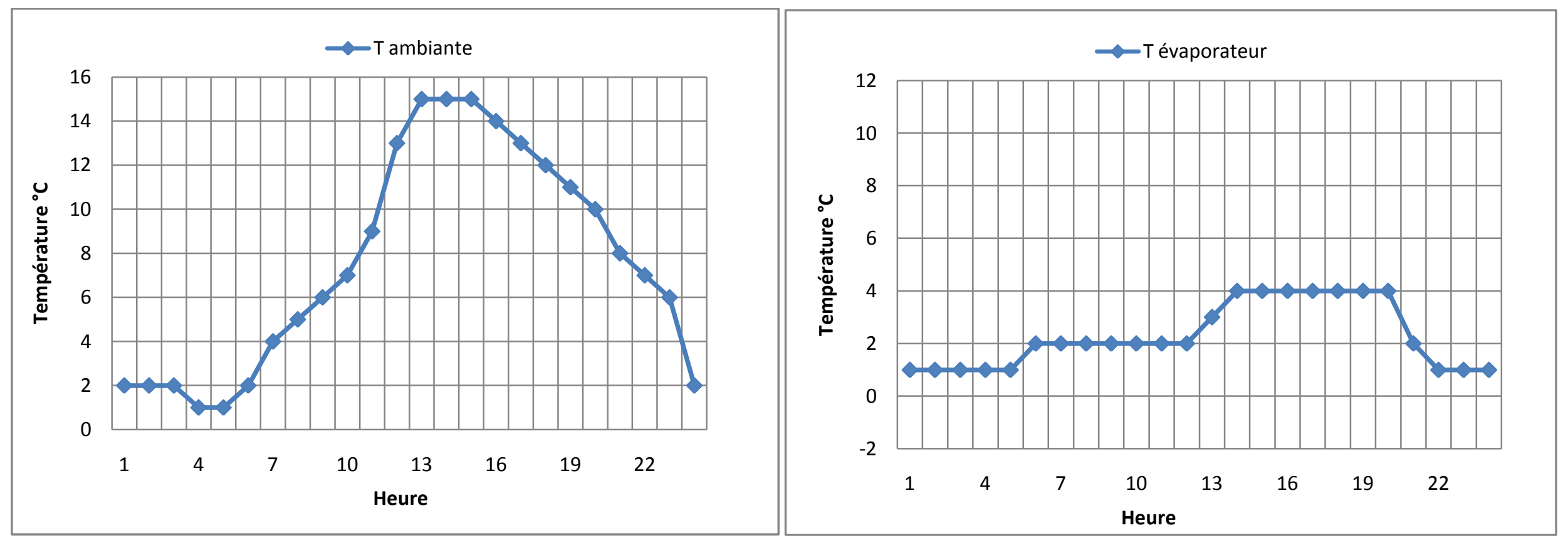




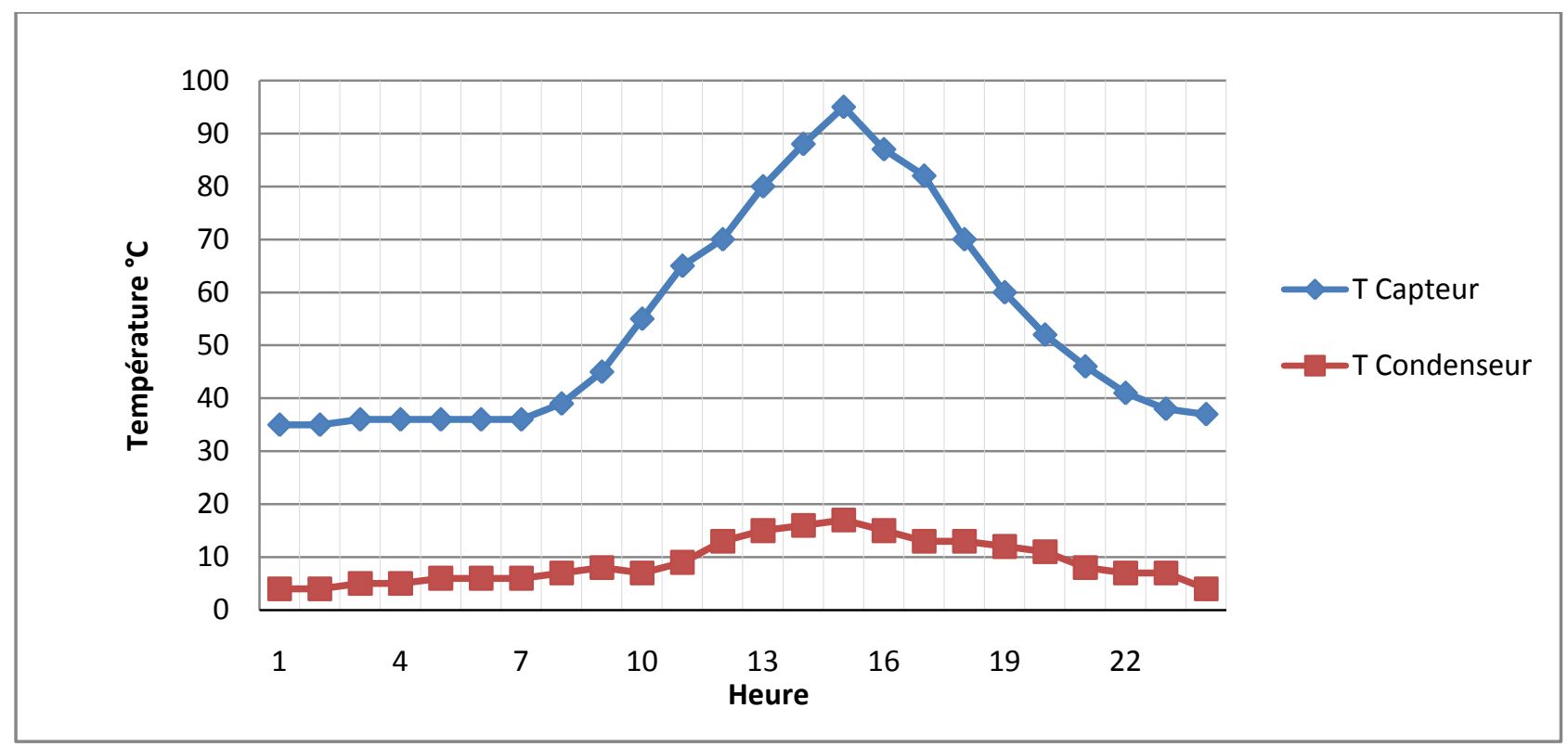

Fig. IV.10 : Températures enregistrées journée du 29 mars 2015
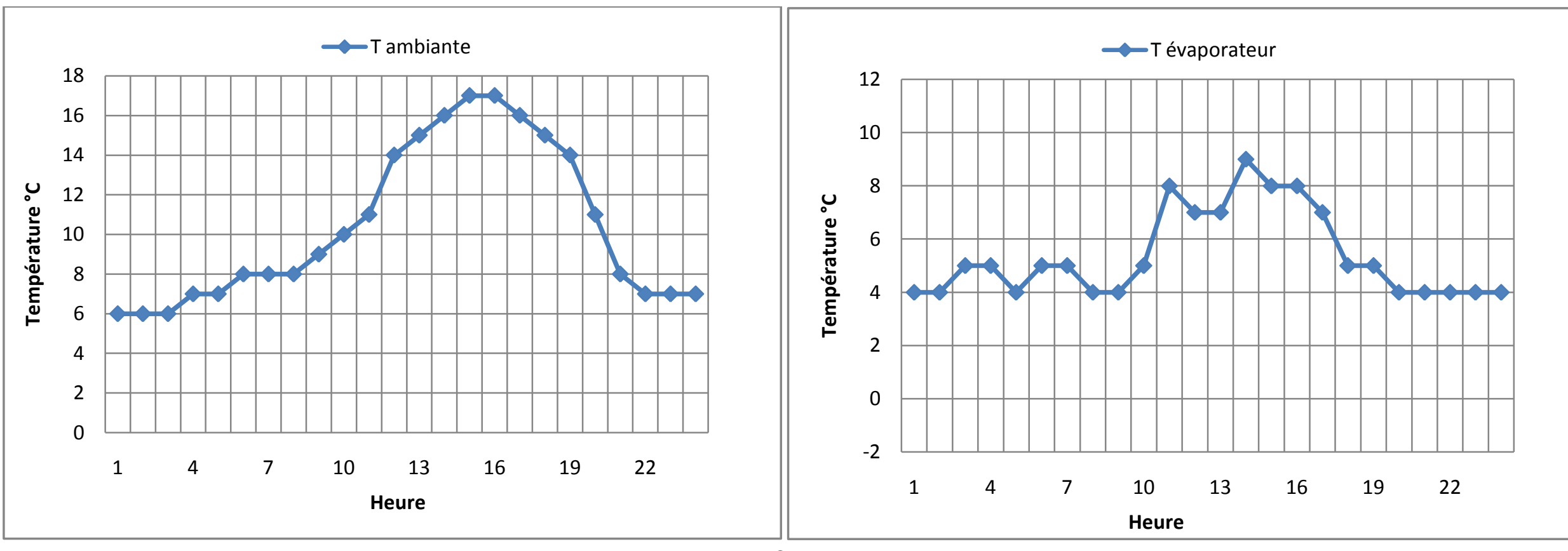

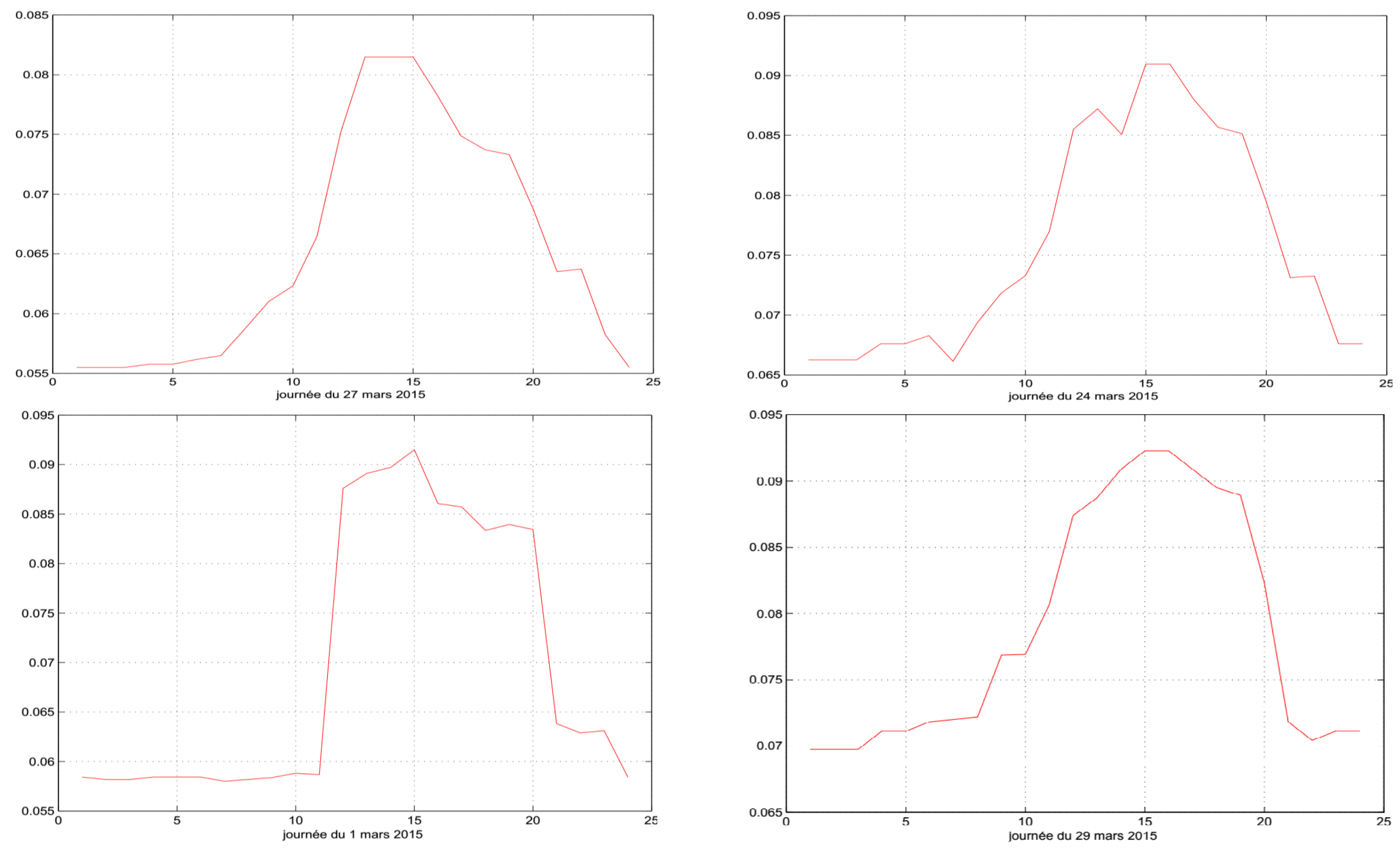

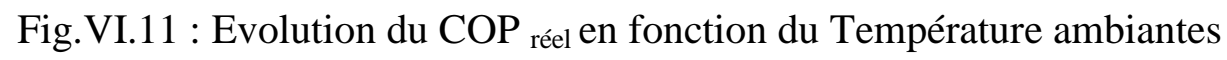




\section{Conclusion générale}

\section{Conclusion générale et perspective}

La machine frigorifique solaire à adsorption présentée dans ce travail est intéressante à plus d'un titre. Elle a fait l'objet de plusieurs études préliminaires récentes afin de la rendre réalisable. Outre l'utilisation de l'énergie solaire, largement disponible et encore mal exploitée dans notre pays, elle peut réaliser des températures de congélation $\left(<-20^{\circ} \mathrm{C}\right)$ avec des sources chaudes à température modérée $\left(70^{\circ} \mathrm{C}\right)$. Elle utilise comme couple le gel de silice et l'eau, un couple 'gratuit' d'une part (eau) et avec un avec prix très raisonnable (gel de silice), de l'autre part.

Parmi les problèmes technologiques rencontrés dans cette machine, le capteur ; il doit réaliser à la fois la captation d'énergie pendant le jour, et surtout assurer le

refroidissement pendant la nuit. $\mathrm{Vu}$ que le refroidissement par rayonnement s'avère insuffisant, la solution retenue réside dans l'adjonction d'ailettes sur la paroi inférieure du capteur, les études de simulation ont montré que la fixation des ailettes triangulaires est la meilleure solution, malgré leur difficulté technologique par rapport aux ailettes rectangulaires simple. 
L'objectif principal de cette étude est de réaliser et expérimenter une machine avec un cout faible, en utilisant de la matière première disponible dans le marché national.

Le but ce n'est pas de développer un prototype innovant mais, d'exploité ce gisement solaire important de notre payé dans la fabrication des congélateurs solaire pour l'intérêt des zone non recouvertes du réseau électrique (conservation des vaccins, médicaments...).

Les résultats expérimentaux trouvés demeure encourageante, ce qui rendre cette étude très utile dans l'amélioration de l'installation expérimentale dans le futur dans les laboratoires de la faculté science et technologie de l'université de Biskra. 


\section{Références bibliographie}

\section{Bibliographie}

[1]. MOUHOUS-CHAOUCHI, Samira. Etude statistique du rayonnement solaire sur un plan incliné. 2012. Thèse de doctorat. univ-bouira. dz; Université Bouira; univ bouira.

[2]. BIALOBRZESKI, Tcheslas. La constitution interne et le rayonnement des étoiles. J. Phys. Radium, 1928, vol. 9, no 8, p. 237-248.

[3]. YAICHE, R. Logiciel de calcul des paramètres astronomiques à l'usage des installations solaires. Revue des Energies Renouvelables ICRESD-07 Tlemcen, 2007, p. 343-348.

[4]. YETTOU, F., MALEK, A., HADDADI, M., et al. Etude comparative de deux modèles de calcul du rayonnement solaire par ciel clair en Algérie. Revue des Energies Renouvelables, 2009, vol. 12, no 2, p. 331-346.

[5]. JOUKOFF, Alexandre. Climatologie du rayonnement solaire. Ciel et terre, 1991, vol. 107, p. 59. 
[6]. PAULIN, Gaston. Simulation de l'énergie solaire au sol. Atmosphere-Ocean, 1980, vol. 18 , no 4, p. 286-303.

[7]. FABRY, Ch et BUISSON, Henri. L'absorption de l'ultra-violet par l'ozone et la limite du spectre solaire. J. Phys. Theor. Appl., 1913, vol. 3, no 1, p. 196-206.

[8]. YAÏCHE, M. R. et BEKKOUCHE, S. M. A. Estimation du rayonnement solaire global en Algérie pour différents types de ciel. Revue des Energies Renouvelables, 2010, vol. 13, no 4, p. 683-695.

[9]. ARORA, Akhilesh et KAUSHIK, S. C. Theoretical analysis of LiBr/H2O absorption refrigeration systems. International Journal of Energy Research, 2009, vol. 33, no 15 , p. 1321-1340.

[10]. KANG, Y. T., KUNUGI, Y., et KASHIWAGI, T. Review of advanced absorption cycles: performance improvement and temperature lift enhancement. International journal of refrigeration, 2000, vol. 23, no 5, p. 388-401.

[11]. HU, Yuanyang, WANG, Liwei, XU, Lu, et al. A two-stage deep freezing chemisorption cycle driven by low-temperature heat source. Frontiers in Energy, 2011, vol. 5, no 3, p. 263-269.

[12]. ILOEJE, O. C. Closed Cycle Solar Refrigeration with the Calcium Chloride system. In : Proceeding of the Workshop on the Physics and Technology of Solar Energy Conversion, Ibandan. 1985.

[13]. FLÉCHON, J., MACHIZAUD, F., BENHAMRNOU, K., et al. Réfrigération solaire par photothermie. Premiers résultats d'un appareil réel. Revue de physique appliquée, 1984, vol. 19, no 8, p. 601-608.

[14]. LE PIERRÈS, Nolwenn. Procédé solaire de production de froid basse température (-28 C) par sorption solide-gaz. 2005. Thèse de doctorat. Université de Perpignan.

[15]. Anyanwu E E., Review of solid adsorption solar refrigerator I," An overview of the refrigeration cycle", Energy Conversion and Management, 44, 301-312, 2003.

[16]. CRITOPH, R. E. Performance limitations of adsorption cycles for solar cooling.Solar energy, 1988, vol. 41, no 1, p. 21-31. 
[17]. DIENG, A. O. et WANG, R. Z. Literature review on solar adsorption technologies for ice-making and air-conditioning purposes and recent developments in solar technology. Renewable and Sustainable Energy Reviews, 2001, vol. 5, no 4, p. 313 342.

[18]. Enibe S.O. et Iloeje O.C., "Transient analysis and performance prediction of a solid absorption solar refrigerator", Solar Energy, 61(1), 42-59, 1997.

[19]. Bansal N K., Blumenburg J., Kavasch H j., Roettinger T., Performance testing and evaluation of solid absorption solar cooling unit, Solar Energy, 61(2), 127-140,1997.

[20]. Li M., Wang R.Z., "A study of the effects of collector and environment parameters on the performance of a solar powered solid adsorption refrigerator", Renewable Energy, 27, 369- 382, 2002.

[21]. Li M., Wang R.Z., Xu Y.X., Wu J.Y., Dieng A.O., "Experimental study on dynamic performance analysis of a flat-plate solar solid -adsorption refrigeration for ice maker", Renewable Energy, 27, 211-221, 2002a.

[22]. Li M., Wang R.Z., luo H.L., Wang L.L., Huang H.B., "Experiments of a solar flat plate hybrid system. With heating and cooling", Applied Thermal Engineering, 22, 1445-1454, 2002b.

[23]. Anyanwu E E., Ezekewe C.I., "Design, construction and test run of a solid adsorption solar refrigerator using activated carbon/methanol as adsorbent/adsorbate pair", Energy Conversion and Management, 44, 2879-2882, 2003.

[24]. Buchter F., Dind P., Pons M., "An experimental solar powered adsorptive refrigerator tested in Burkina-Faso", International Journal of Refrigeration, 26, 79-86, 2003.

[25]. Hilbrand C., Dind P., Pons M., Buchter F., "A new solar powered adsorption refrigerator with high performance", Solar Energy, 77, 311-318, 2004.

[26]. TAMAINOT-TELTO, Z. et CRITOPH, R. E. Adsorption refrigerator using monolithic carbon-ammonia pair. International Journal of Refrigeration, 1997, vol. 20, no 2 , p. 146-155. 
[27]. Erhard A., Hahne E., "test and simulation of a solar-powered absorption cooling machine", solar Energy, 59(4-6), 155-162, 1997.

[28]. Erhard A., Spindler K Hahne E., "test and simulation of a solar-powered absorption cooling machine, International Journal of Refrigeration", 21(2), 133141.1998.

[29]. Berlitz T., Plank H., Ziegler F., Kahn R., "An ammonia-water absorption refrigerator with a large temperature lift for combined heating and cooling", International Journal of Refrigeration, 21(3), 219-229.1998.

[30]. Best R., Pilatowsky I., "Solar assisted cooling with sorption systems: status of the research in Mexica and Latin America", International Journal of Refrigeration, 21(2), 100-115.1998.

[31]. Wang, R. Z., \& Oliveira, R. G. (2006). Adsorption refrigeration-an efficient way to make good use of waste heat and solar energy. Progress in Energy and Combustion Science, 32(4), 424-458.

[32]. BOUGARD, J. Thermodynamical and technical problems in solar adsorption refrigeration. In : Institut international du froid, commissions B1, B2, E1 \& E2. Réunion. 1986. p. 25-33.

[33]. SING, Kenneth SW et GREGG, S. J. Adsorption, surface area and porosity.Academic Press, London, 1982, p. 1-5.

[34]. WATANABE, F., KOZUKA, J., KUMITA, M., et al. Adsorption equilibrium of silica gel, active carbon/water vapor, ethanol and applicability of combination of those adsorbent/adsorbate to adsorption heat pump. Kagaku Kogaku Ronbunshu, 1993, vol. 19, p. 1165-1165.

[35]. SECULA, Marius Sebastian, CAGNON, Benoît, FAUDUET, Henri, et al.Modification des proprietes poreuses des charbons actifs et de gels de silice par impregnation au chlorure de calcium. In : Le 6ème Colloque Franco-Roumain de Chimie Appliquée (COFrRoCA). 2010. 
[36]. WONGSUWAN, W., KUMAR, S., NEVEU, P., et al. A review of chemical heat pump technology and applications. Applied Thermal Engineering, 2001, vol. 21, no 15 , p. 1489-1519.

[37]. BOUGARD, J. et VERONIKIS, G. Adsorbeur modulaire pour machine frigorifique solaire charbon actif-ammoniac. In : Symposium "Le froid a sorption solide", paris. 1992. p. 282-287.

[38]. MERMOND, Y., FEIDT, M., et MARVILLET, C. Propriétés thermodynamiques et physiques des mélanges de fluides frigorigènes et d'huiles. International journal of refrigeration, 1999, vol. 22, no 7, p. 569-579

[39]. S. T. GREGG and K. W. SING, adsorption surface area and porosity, 2nd ED. Academic press, London, 1977.

[40]. Chekirou wassila, Etude et analyse d'une machine frigorifique solaire à adsorption, Thèse de doctorat option photo-thermique, U Mentouri Constantine $03 / 06 / 2008$

[41]. BOUGARD, J. Thermodynamical and technical problems in solar adsorption refrigeration. In : Institut international du froid, commissions B1, B2, E1 \& E2. Réunion. 1986. p. 25-33.

[42]. Chems. Eddine Chitour, physico-chimie de surface, Vol 2, les interfaces gazsolide et liquide-solide. U Mentouri Constantine 2001.

[43]. ELM'CHAOURL, A. isothermes et isobares d'adsorption-desorption. 1990. Thèse de doctorat. These \$ ci.. Univ. de Bourgogne. Diion, France 16 p.

[44]. R.T.Yang, Gas separation by Adsorption processes. Butterworths, london, 1987.

[45]. GRILLET, Yves, ROUQUEROL, Françoise, et ROUQUEROL, Jean. Etude de l'adsorption physique des gaz par une procédure continue. I. Application à la détermination des surfaces spécifiques d'adsorbants non poreux et mésoporeux. Journal of Chemical Physic, 1977, vol. 74, p. 179-182.

[46]. L. M. SUN, F. Meunier, Adsorption. Aspects théoriques, J 2730 Techniques de l'ingénieur, 2003. 
[47]. S, Brunauer, P. H. Emmett, E. Teller, Adsorption of gases in multimolecular layers, J. Am. Chem. Soc, volume 60, George Washingtounn unniversiti. 1938.

[48]. M. M. Dubinin, Microporous structures and adsorption properties of carbonaceous adsorbents, Carbon, volume 21, 1983.

[49]. J. H. De BOER, dynamical character of adsorption, oxford at the Clarendon press, 1968.

[50]. G. EMSHWILLER, Equilibres en solution-Phénomènes de surface, tome 11, chimie physique, presses universitaires de France, 1951.

[51]. M. M. Dubinin, the potential theory of adsorption of gases and vapors for adsorbents with energetically nonuiform surfaces, Chem. Rev, volume 60, 1960.

[52]. N. Hebbir et N. Labed, 'Optimisation des Echanges Thermiques par Convection Naturelle d'un Capteur Régénérateur d'une Machine Frigorifique Solaire', Séminaire International sur la Physique Energétique, SIPE'8, Béchar, Algérie, Novembre 2006.

[53]. HEBBIR, N. et LABED, N. Simulation et dopage des échanges par convection naturelle d'un capteur régénérateur d'une machine frigorifiques solaire.

[54]. DE LIETO VOLLARO, Andrea, GRIGNAFFINI, Stefano, et GUGLIERMETTI, Franco. Optimum design of vertical rectangular fin arrays. International journal of thermal sciences, 1999, vol. 38, no 6, p. 525-529.

[55]. LÉVÊQUE, André. Les Lois de la transmission de chaleur par convection, par André Lévêque,.. Dunod, 1928.

[56]. Laboratoire d'Etudes Thermiques, "Dopage des échanges par convection naturelle en cycle nocturne", Rapport de projet PRI 6.1 Froid Solaire «, Poitiers 2004.

[57]. DELGADO, R., CHOISIER, A., GRENIER, Ph, et al. Etude du cycle intermittent charbon actif-méthanol en vue de la réalisation d'une machine à fabriquer de la glace fonctionnant à l'énergie solaire. In : L'Énergie solaire pour le froid et le conditionnement d'air. Institut international du froid. Commissions E1-E2. Réunions. 1982. p. 185-191. 
[58]. PASSOS, Evandro Ferreira. Etude des couples charbon actif/méthanol et de leur application à la réfrigération solaire. 1986.

[59]. MHIRI, Foued et EL GOLLI, Salem. Etude d'un refrigerateur solaire a adsorption solide avec le couple charbon actif-methanol. Revue générale de thermique, 1996, vol. 35, no 412, p. 269-277.

[60]. LEMMINI, F., BURET-BAHRAOUI, J., PONS, M., et al. Simulation des performances d'un réfrigérateur solaire à adsorption: 1. comparaison des performances pour deux types de charbon actif. International journal of refrigeration, 1992, vol. 15, no 3 , p. 159-167.

[61]. BOUBAKRI, Abdesselem. Détermination des caractéristiques thermodynamiques du couple charbon actif $\mathrm{AC}$ 35-méthanol et étude de son application à la réfrigération solaire. 1985. Thèse de doctorat.

[62]. ERROUGANI, Abdelmoussehel. Fabrication et expérimentation d'un réfrigérateur solaire a adsorption utilisant le couple charbon actif-méthanol dans le site de Rabat. 2007.

[63]. GUILLEMINOT, J. J. et MEUNIER, F. Etude expérimentale d'une glacière solaire utilisant le cycle zéolithe 13X-eau. Rev. Gen. Therm. Fr, 1981, vol. 239, p. 825-834.

[64]. DUPONT, M., GUILLEMINOT, J. J., MEUNIER, F., et al. Etude de glacières solaires utilisant le cycle intermittent jour-nuit zeolithe 13x-eau en climat tempere et en climat tropical. In : L'Énergie solaire pour le froid et le conditionnement d'air. Institut international du froid. Commissions E1-E2. Réunions. 1982. p. 193-200.

[65]. MARMOTTANT, B., MHIMID, A., EL GOLLI, S., et al. Installation de réfrigération solaire à adsorption: expérimentation et modélisation. Revue générale de thermique, 1992, vol. 31, no 362, p. 97-105.

[66]. TCHERNEV, T. I. Solar air conditioning and refrigeration systems utilizing zeolites. In : L'Énergie solaire pour le froid et le conditionnement d'air. Institut international du froid. Commissions E1-E2. Réunions. 1982. p. 209-215. 
[67]. MHIMID, Abdallah, JEMNI, Abdelmajid, et NASRALLAH, Sassi Ben. Etude théorique des transferts couples de chaleur et de masse lors de la désorption du couple Zéolithe 13X-eau. Revue générale de thermique, 1997, vol. 36, no 9, p. 697-706.

[68]. GRENIER, Ph, GUILLEMINOT, J. J., MESTER, M., et al. Entrepôt frigorifique solaire à cycle zéolithe-eau. J. Int. Transf. Hélioth.(Monastir), 1983.

[69]. A. Sakoda, and M. Suzuki., Fundamental study on solar powered engineering Japan, Vol 17, pp 52-57, 1984.

[70]. DIND Philippe, HILDBRAND Catherine, CHERBUIN Olivier, MAYOR Julien. 2005. La réfrigération solaire à adsorption. HES-SO : Projet Gerbert Rüf Stiftung. Yverdon-les-Bains, Suisse. 
$\underline{\text { Annexe \& publications }}$ 


\title{
SIMULATION ET DOPAGE DES ECHANGES PAR CONVECTION NATURELLE D'UN CAPTEUR RÉGÉNÉRATEUR D'UNE MACHINE FRIGORIFIQUE SOLAIRE À ADSORPTIONÀ AILETTE DE TYPE RECTANGULAIRE ET TRIANGULAIRE
}

\author{
HEBBIR NACER, CHEBBAH M-SAID, GHODBAN HATEM \\ Université de Biskra, BP 145 Biskra, 07000, Algérie \\ n.hebbir@univ-biskra.dz
}

\begin{abstract}
RESUME
Le besoin en froid dans le secteur de l'habitat individuel ou collectif est en forte augmentation et l'une des applications qui répond aux exigences environnementales est l'utilisation des machines frigorifiques à adsorption solaire. Celles-ci sont équipées par des capteurs régénérateurs. Ils ont comme rôle; de capter l'énergie solaire le jour (phase diurne), et évacuer la chaleur du réacteur en phase nocturne. Les premières évaluations du projet montrent que le problème se trouve au niveau de la phase de production de froid (nuit). La solution proposée est l'adjonction des ailettes rectangulaires ou triangulaire sur la paroi du capteur. Notre objectif est de faire une étude comparative entre ces deux types d'ailette, afin de trouver les dimensions géométriques optimales des ailettes à implanter sur le capteur.
\end{abstract}

\begin{abstract}
The need of cooling in the individual or collective housing sector is rising sharply, one of these applications which gives the answer to environmental requirements is the use of solar adsorption refrigeration machinery. They are equipped with regenerators' sensors. Their role is to capture solar energy during the day (diurnal phase), and remove heat from the reactor in the night period. The first evaluations of the project show that the problem is connected to the production phase of cold (night).The proposed solution is the use of rectangular or triangular fins on the side of the sensor. Our goal here is to study this type of fins, in order to find the optimal geometricdimension of the fins implanted on the sensor.
\end{abstract}

MOTS CLES: Machine frigorifique solaire - Convection naturelle - Capteur solaire - Ailettes rectangulaires.

\section{INTRODUCTION}

Avec l'accroissement de la population et ledéveloppement industriel, surtout dans les régionspétrolières, les besoins de l'Algérie en froid sont de plus enplus importants. Le recours aux énergies renouvelables,surtout solaire, devient plus que nécessaire pour répondre àces besoins.

L'un des procédés de production de froid par énergiesolaire est le procédé thermochimique à adsorption.Plusieurs laboratoires français sont impliqués pour laconception d'une nouvelle machine frigorifique solaire àadsorption. Elle devra réaliser une température de $\left(-30^{\circ} \mathrm{C}\right)$ etutilisera une source solaire basse température de $70^{\circ} \mathrm{Ccontrairement}$ au machines existantes actuelles.

Les premières évaluations du projet ont montré que lescontraintes de dimensionnements les plus fortes se situaientnon pas au niveau de captation de l'énergie solaire, mais aucontraire, au niveau du refroidissement du réacteur dans laphase nocturne de production.

\section{PROBLÉMATIQUE}

L'objectif de ce travail, est de concevoir un capteurperformant le jour, et possédant une fonction de radiateur,durant la phase nocturne de régénération, le flux à évacuerest de $250 \mathrm{~W} / \mathrm{m} 2$ à travers une surface de $10 \mathrm{~m} 2$, soit uncoefficient d'échange apparent de l'ordre de happ = Wm-2k-1.Une étude préliminaire d'optimisation à déjà permis de fixerles dimensions des ailettes à implanter sur la partie basse ducapteur [3].

L'objectif principal est de simuler le dopage des échanges thermiques par convection naturelleau niveau des ailettes. Il faut notamment vérifier que lecapteur choisi est tout à fait capable d'évacuer la quantitéde chaleur donnée pendant le temps fixé. Nous avons utilisé le logiciel FLUENT qui est largement utilisé dans le domaine dela mécanique des fluides et de la thermique. 


\section{DOMAINE D'ÉTUDE}

Le capteur aileté par sa face inférieure présente unepériodicité régulière. On dispose donc de deux plans desymétrie. Le domaine d'étude sera réduit à un motifélémentaire englobant une demi-ailette et un demiécartement. Pour notre étude on propose d'étudier deux types d'ailette soit rectangulaire et triangulaire.
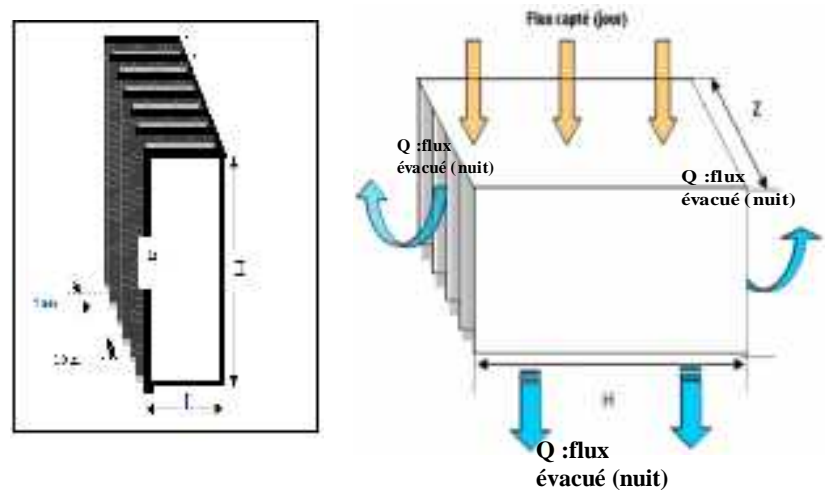

Figure 1a, 1b : Vue en perspective du capteur muni d'ailettes rectangulaire

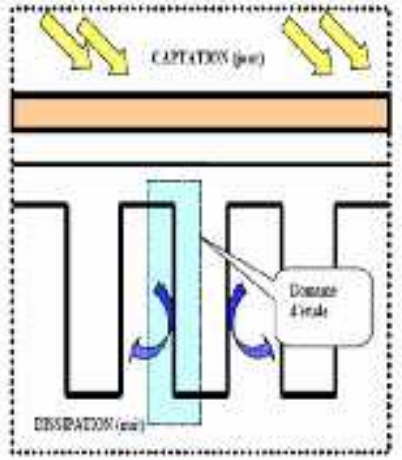

ler Cas : ailette rectangulaire

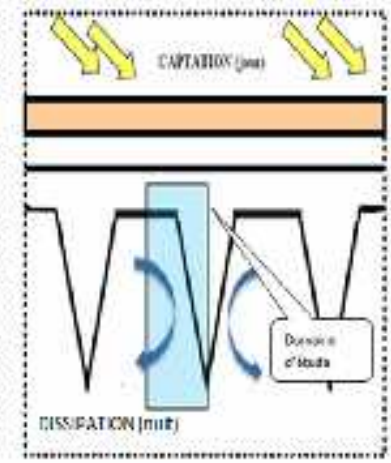

2eme Cas : ailette triangulaire

Figure 2 : Vue de dessus du domaine d'étude

\section{CONDITIONS AUX LIMITES}

Avec le lexique du code, nous avons :

- Les faces avant et arrière sont caractérisées par unecondition de symétrie (symmetry).

- La paroi du capteur est associée à une condition detempérature imposée $\mathrm{Tp}=70^{\circ} \mathrm{C}$.

- La conduction dans l'ailette est prise en compte,autrement dit celle-ci est considéré comme élémentsolide "solid", de conductivité thermique $\lambda=204.4 \mathrm{Wm}-1 \mathrm{~K}-1$, ce qui correspond à laconductivité thermique de l'aluminium.

- Les frontières ouvertes du domainecaractérisées par les conditions aux limites :
1. Velocity inlet : Cette condition permet d'imposer surune surface une vitesse et une température du fluideentrant, la vitesse est estimée à $0.03 \mathrm{~m} / \mathrm{s}$.

2. Outflow : Cette condition est traduite le fait qu'onne connaît pas les paramètres (vitesse et pression)du fluide sortant.

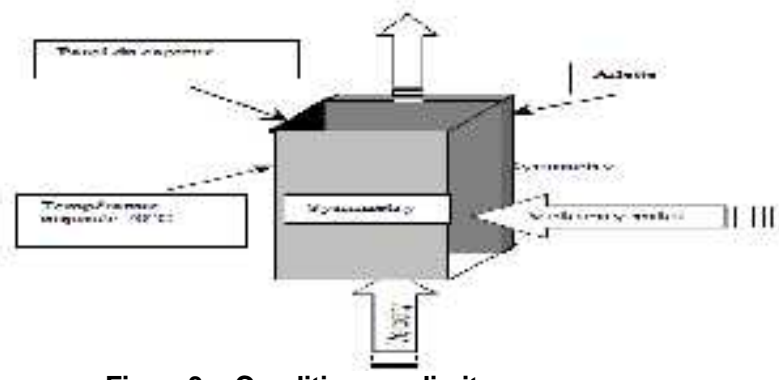

Figure3 : Condition aux limites

\section{MAILLAGE}

L'écoulement de l'air autours des ailettes en convectionnaturelle est supposé laminaire en régime stationnaire selonun modèle isolé en formulation implicite. Le fluide estsupposé incompressible sous l'hypothèse de Boussinesq.

Etant donné que le domaine d'étude ne présente pas de singularité particulière, ce maillage est généré grâce aulogiciel 'GAMBIT', Les pas d'espace suivant ; $x, y$, zsont respectivement égale à $4 \mathrm{~mm}, 50 \mathrm{~mm}, 1 \mathrm{~mm}$.

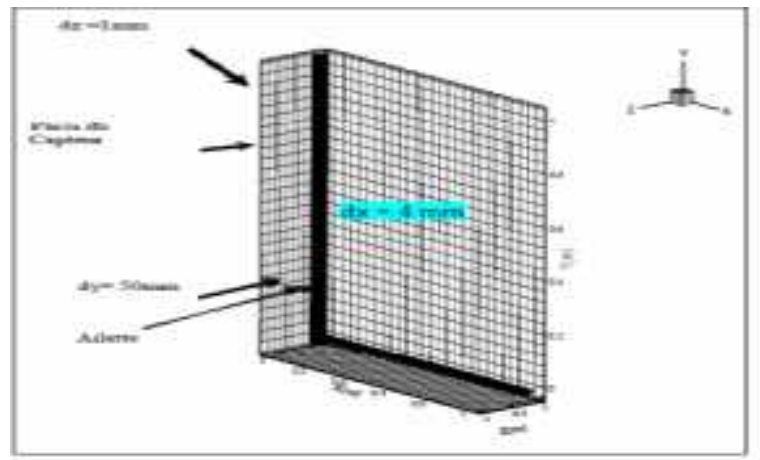

Figure 4 : maillage du domine

\section{RÉSULTAT}

Rappelons que la problématique consiste à biendimensionner les ailettes du capteur pour dégager la quantitéde chaleur fixée pendant un temps limité. Pour la présentation des résultats obtenus, nous avons choisi dereprésenter les isothermes et les profils de vitesses.

Les profils de vitesses sur la (fig. 5) sont calculés pourune longueur de l'ailette égale à $8 \mathrm{~cm}$ s'échauffe etune force ascensionnelle est créée permettant à l'aird'accélérer son 
mouvement vers le haut. Le Coefficient d'échange est d'autant plus fort que la vitesse de l'air estplus importante.

On a considéré que la vitesse de l'air à l'entrée (coté baset avant) est nulle. A la sortie du domaine, la vitesse atteintson maximum avec une valeur importante (environ $0.91 \mathrm{~m} / \mathrm{s}$ pour les ailettes triangulaire et $0.52 \mathrm{~m} / \mathrm{s}$ pour les ailettes rectangulaire). Ce qui traduit un échauffement important et donc unebonne convection.

Sur les figures 6, on propose les profils de températures sur les plans de symétrie avant perpendiculaire par rapport au capteur, On voit clairement le développement de la couche limite de convection naturelle, ce qui traduit bien l'effet de refroidissement du capteur. On remarque aussi que la majorité des échanges sont assuré par la moitié de l'ailette.

A la lecture des résultats obtenus, on peut dire qu'ilest tout à fait possible de réaliser, avec des ailettes triangulaire ou rectangulaire, l'échange thermique souhaité parconvection naturelle uniquement pendant le temps fixé. Ces résultats montrent que les ailettes triangulaires ont des performances meilleures que celles des ailettes rectangulaires.

Ceci rejoint les conclusions de l'étude préliminaireeffectuée au LET [1] même si, pour notre calcul, on aconsidéré les conditions climatiques de la ville de biskra.
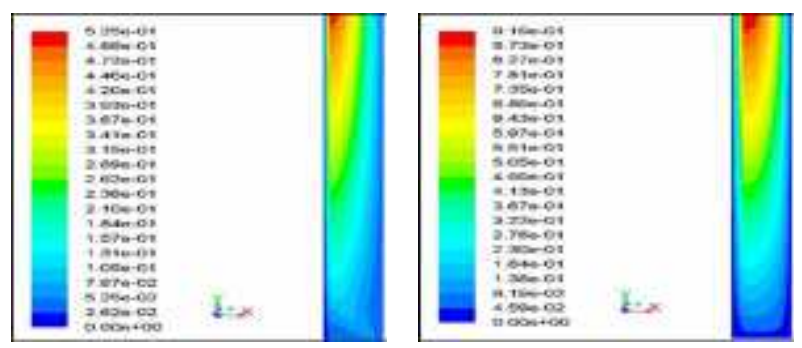

1er Cas : ailette rectangulaire 2emeCas : ailette triangulaire Figure 5 : profil de vitesse de l'air (plan de symétrie) en $\mathrm{m} / \mathrm{s}$

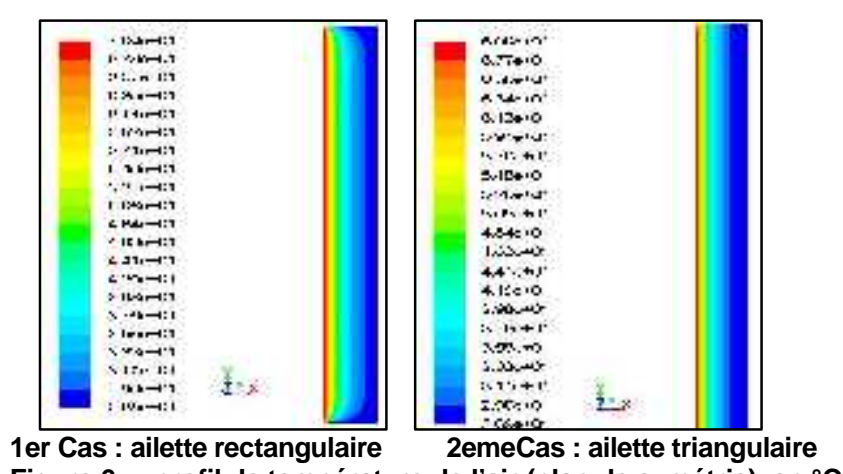

Figure 6 : profil de température de l'air (plan de symétrie) en ${ }^{\circ} \mathrm{C}$

\section{SENSIBILITÉ AU MAILLAGE}

Afin de confirmer les résultats trouvés, nous avonseffectué un changement de paramètres de maillage. L'étude de sensibilité au maillage a été fait par le jeudes paramètres suivants $: \mathrm{i}=16 \mathrm{~cm}, \mathrm{e}=4 \mathrm{~mm}$ et $\mathrm{La}=8 \mathrm{~cm}$.

La figure 9 représente l'influence de l'affinage dumaillage sur le profil de température et la composanteverticale de la vitesse dans le plan de symétrie avant à mi-hauteur du capteur.

La courbe noire est celle du maillage de base alors quecelle de couleur violet est celle obtenue avec le maillage fin(la moitié du pas) La jaune est celle du maillage large (ledouble du pas de base).

On voit clairement que les résultats trouvés neprésentent qu'une légère sensibilité au maillage, on peutdonc conclure que les résultats de la simulation sontacceptables.

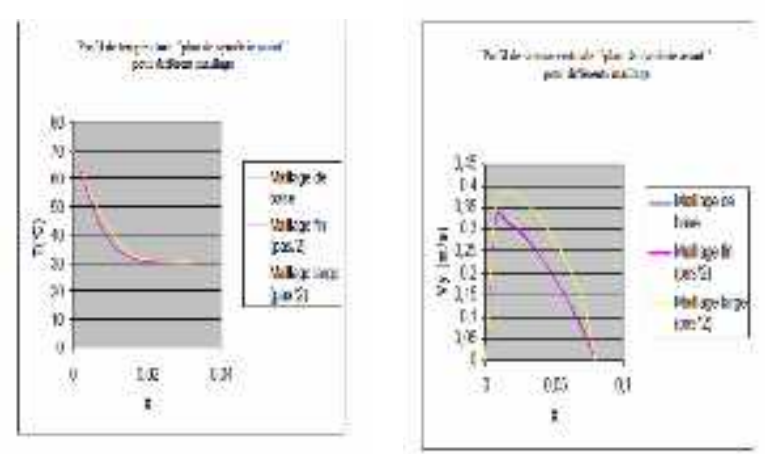

Figure 9 : sensibilité aux maillages

\section{CONCLUSION}

La comparaison des résultats des deux types d'ailette, montre que les choix d'implanter des ailettes triangulaires sur le capteur donnentdes résultats meilleurs, mais malheureusement la construction de ce type d'ailette reste difficile, donc on a opté pour le choix des ailettes rectangulaire .

En conclusion à cette étude, nous pouvons dire que lechoix d'ailette rectangulaire simple est tout à faitsatisfaisant. Le flux à évacuerpour permettre larégénération du fluide frigorigène est suffisamment dissipépar la partie basse du capteur-régénérateur. En plus, nosrésultats sont comparables avec ceux effectué au LET [1].

\section{REFERENCES}

[1] LET, rapport de projet PRI 6.1 Froid solaire, Poitiers (2004).

[2] Andréa sz lieto vollaro, Stefano Grignaffini, franco gugliermitti"optimum design of vertical rectangular fin arrays" Int. J. Sc. 38. Pp525 -529, 1999.

[3] N.HEBBIR, N. LABED « optimisation des échanges thermique parconvection naturelle d'un capteur régénérateur d'une machinefrigorifique solaire ». 
SIPE8 Nov.2006, Béchar. Algérie.

[4] Nolwenn Le Pierres, STITOU D., MAZET N. « Conception d'unprocédé thermochimique de congélation solaire à partir de chaleurbasse température » IMP (Institut de Science et Génie des Matériauxet Procédés), UPR CNRS 8521 Rambla de la Thermodynamique,Tecnosud, 66100 Perpignan (France).
[5] Nolwenn Le Pierres, «Procédé solaire de production de froid basetempérature $\left(-28^{\circ} \mathrm{C}\right.$ par sorption solidegaz ", thèse soutenue en septembre 2005, Université de Perpignan, thèse soutenue en Sep2005.

[6] André B. De Vrient, «La transmission de la chaleur », Edition, Gaëtan Morin, 1984.

[7] CNRS, rapport final d'activité 2002/2004, GAT 6 : Habitat, « Congélation solaire $-30^{\circ} \mathrm{Cà} \mathrm{par} \mathrm{procédé}$ thermochimique à partird'une source thermique basse température $\left(70^{\circ} \mathrm{C}\right)$. 


\begin{tabular}{|c|c|}
\hline & SEMINAIRE NATIONAL SUR LES SYSTEMES ENERGETIQUES HYBRIDES \\
$10-11$ Novembre 2015 \\
Université de Ghardaïa \\
Faculté des Sciences et de la Technologie
\end{tabular}

\title{
Etude des échanges par convection naturelle d'un capteur -régénérateur d'une machine frigorifique solaire à adsorption
}

\author{
HEBBIR Nacer ${ }^{1}$, CHEBBAH M-Said, Hatem Ghodban \\ 'Laboratoire Génie énergétique et matériauX«LGEM» université de Biskra
}

habnacer@gmail.dz

\begin{abstract}
Résumé- Le besoin en froid dans le secteur de l'habitat individuel ou collectif est en forte augmentation, l'une des applications (qui répond aux exigences environnementales) est l'utilisation des machines frigorifiques à adsorption solaire. Celles-ci sont équipées par des capteurs régénérateurs. Ils ont comme rôle; de capter l'énergie solaire le jour (phase diurne), et évacuer la chaleur du réacteur en phase nocturne. Les premières évaluations du projet montrent que le problème se trouve au niveau de la phase de production de froid (nuit). La solution proposée est l'adjonction des ailettes rectangulaires sur la paroi du capteur. Notre objectif est de faire une étude de ce type d'ailette, afin de trouver les dimensions géométriques optimales des ailettes à implanter sur le capteur.
\end{abstract}

Mots clés: Machine frigorifique solaire - Convection naturelle Capteur solaire - Ailettes rectangulaires.

\section{1- INTRODUCTION}

Le recours aux énergies renouvelables comme solution de substitution aux énergies fossiles devient de plus en plus indispensable. Le réchauffement climatique terrestre et le tarissement progressif des ressources sont des facteurs déterminants.

L'utilisation de l'énergie solaire reste limitée, surtout dans les pays du sud. Des problèmes liés à la maîtrise technologique de captation et à la transformation de cette énergie constituent un obstacle considérable à sa généralisation malgré sa disponibilité. Les avantages de ce type d'énergie ne sont plus à démontrer. Hormis, sa disponibilité et sa « gratuité », elle reste utilisable même dans les régions rurales ou sahariennes les plus isolées, et donc non raccordées au réseau électrique conventionnel.
Parmi les procédés de transformation thermique de l'énergie solaire, la réfrigération solaire. Le froid produit servira à la climatisation des locaux et au stockage des denrées alimentaires et des produits pharmaceutiques.

L'Algérie est un pays où le potentiel solaire est très important particulièrement dans les régions du sud. Avec l'accroissement de la population et le développement des infrastructures industrielles, surtout pétrolières, les besoins en froid (positif et négatif) sont en forte augmentation.

Plusieurs procédés de production de froid par énergie solaire sont disponibles. Les procédés thermochimiques, à absorption ou adsorption sont les plus utilisé. Leur principe est simple : Il repose sur la solubilité d'un gaz, ou d'un solide, dans un liquide qui est fonction de la pression et de la température. C'est un domaine de recherche très vaste et qui nécessite d'importants moyens expérimentaux et de calcul. Il est à signaler aussi, que le marché du solaire est très promoteur pour les années à venir. Les grandes entreprises internationales sont en concurrence pour le développement de machines avec un prix raisonnable du $\mathrm{kW}$.

Plusieurs travaux dans le domaine de réfrigération par adsorption ont été réalisés. pour concevoir, étudier la faisabilité et expérimenter un procédé de sorption solide/gaz permettant une production de froid inférieure à $22^{\circ} \mathrm{C}$ en utilisant une source thermique à bas niveau de température (de l'ordre de 60 à $70^{\circ} \mathrm{C}$ ). L'application principale est la congélation de denrées alimentaires dans les zones dépourvues de réseau électrique et dans l'habitat (chambre froide intégrée). L'originalité de ce projet est que la source chaude utilisée sera uniquement solaire avec une 


\begin{tabular}{|c|c|}
\hline & SEMINAIRE NATIONAL SUR LES SYSTEMES ENERGETIQUES HYBRIDES \\
$10-11$ Novembre 2015 \\
Université de Ghardaïa \\
Faculté des Sciences et de la Technologie
\end{tabular}

température relativement faible $\left(70^{\circ} \mathrm{C}\right)$. Il n'existe actuellement pas de procédés solaires de congélation requérant ce faible niveau de température de source chaude.

Les objectifs scientifiques de ce projet portent sur les interactions entre une source intermittente (solaire), un procédé à fonctionnement cyclique et une enceinte de congélation à $-22^{\circ} \mathrm{C}$ requérant une faible fluctuation de son niveau de température (critère de qualité de conservation de denrées). L'objectif n'est seulement pas de développer un procédé innovant de congélation solaire, mais aussi de proposer un système qui répond aux exigences socioéconomique; un coût global faible, une simplicité technologique (systèmes sans vanne, auto adaptif aux conditions extérieures)

On se limite dans cette étude au dimensionnement et la modélisation d'un nouveau capteur solaire à fonctionnement cyclique altérant une phase de captation d'énergie et une phase de dégagement de chaleur. Les études préliminaires ont montrées que la partie «captation » ne présente pas de difficulté particulière. Le problème se situe essentiellement donc, dans la partie « radiateur » du capteur. Il s'agit donc de répondre à un « cahier de charges » bien défini pour évacuer une quantité fixée de chaleur pendant un temps déterminé. La modélisation des échanges thermiques, essentiellement par convection naturelle, pour différentes formes du capteur constitue l'essentiel de cette étude.

Dans la réfrigération solaire entre $0-10^{\circ} \mathrm{C}$ quelques systèmes seulement sont capables de produire du froid négatif, ils utilisent l'ammoniac, qui est totalement neutre pour l'environnement, comme fluide de travail, ce sont des systèmes à sorption liquide/gaz : NH3/H2O, ou solide/gaz : $\mathrm{SrCl} 2 / \mathrm{NH} 3, \mathrm{CaCl} 2 \mathrm{NH} 3$, Ces systèmes permettent une production de froid à $-10^{\circ} \mathrm{C}$ (glace solaire), leur inconvénient majeur réside dans la nécessite d'une température de source chaude minimale de $120^{\circ} \mathrm{C}$, or ce niveau de température demande une technologie très avancées ce qui rend le coût total de l'installation trop élevé.

Dans le domaine de la congélation solaire $\left(\mathrm{t}^{\circ}-20^{\circ} \mathrm{C}\right)$, à notre connaissance, aucun système mis à part les systèmes à compression, n'a été testé, du fait des températures très élevées requises par la source chaude.

L'intérêt d'utiliser des capteurs solaires basses températures est d'obtenir des rendements de captation élevés de l'ordre de $50 \%$ à $70 \%$, ainsi le coefficient de performance solaire (SCOP) de l'installation de congélation solaire, rapport de l'énergie frigorifique produite à l'énergie solaire captée pourrait atteindre une valeur de10 à 20\%, supérieure à celle des systèmes actuellement développés oscillants entre 8 et $20 \%$.

Plusieurs laboratoires internationaux sont impliqués pour la conception d'une nouvelle machine frigorifique solaire à adsorption. Elle devra réaliser une température de $\left(-30^{\circ}\right)$ et utilisera une source solaire basse température de $70^{\circ} \mathrm{C}$ contrairement aux machines actuelles, les premières évaluations du projet ont montré que les contraintes de dimensionnement les plus fortes se situaient non pas au niveau de la captation de l'énergie solaire, mais au contraire, au niveau du refroidissement du réacteur dans la phase nocturne de production de froid. [1] [2].

\section{2- PROBLEMATIQUE}

La faisabilité du projet de froid solaire est essentiellement basé sur la capacité des capteurs, d'une part, à emmagasiner suffisamment d'énergie durant la journée ceci afin de desorber le gaz et, d'autre part, à évacuer suffisamment la nuit d'adsorber le gaz et en conséquence produire le froid.

L'objectif à remplir durant le jour peut facilement être atteint avec des capteurs conventionnels. Cependant assurer l'évacuation de la chaleur durant la nuit est beaucoup plus délicat. L'objectif de cette étude, est de concevoir un capteur performant le jour, et possédant une fonction de radiateur la nuit. [3]

En cycle nocturne l'énergie à évacuer est de $12 \mathrm{Kw}$ en 5 heures [4], ce qui correspond à une puissance de $2.5 \mathrm{kw}$. Choisissant une surface raisonnable du capteur de $10 \mathrm{~m}^{2}$, la densité de flux à évacuer est de $250 \mathrm{~W} / \mathrm{m}^{2}$. Soit un coefficient d'échange apparent de l'ordre de $25 \mathrm{Wm}^{-2} \mathrm{~K}^{-1}$.

On choisira, dans un premier temps, une plaque plane verticale sur laquelle va se développer l'écoulement de convection naturelle avec une hauteur de $1 \mathrm{~m}$. Elle sera inclinée d'un angle $\alpha$ par rapport à la verticale pour améliorer le rendement de captation en cycle diurne.

A partir des lois de convection naturelle sur une plaque plane verticale en régime laminaire [4], il est possible d'évaluer le coefficient d'échange résultant, le nombre de 


\begin{tabular}{|c|c|}
\hline & SEMINAIRE NATIONAL SUR LES SYSTEMES ENERGETIQUES HYBRIDES \\
$10-11$ Novembre 2015 \\
Université de Ghardaïa \\
Faculté des Sciences et de la Technologie
\end{tabular}

Nusselt bâti sur la hauteur $\mathrm{H}$ de la plaque s'exprime par la relation :

$$
\mathrm{Nu}_{\mathrm{H}}=\mathrm{h}_{0} \mathrm{H} / \lambda=0.59 \mathrm{Ra}_{\mathrm{H}}{ }^{2} / 4
$$

$\boldsymbol{R} \boldsymbol{a}_{\boldsymbol{H}}$ est le nombre de Rayleigh bâti sur la hauteur H :

$$
\mathrm{Ra}_{\mathrm{H}}=\frac{\mathrm{g} \beta \cos \alpha \Delta \mathrm{TH}^{3}}{(\alpha \mathrm{u})}
$$

Les propriétés thermo physiques de l'air sont prises à la température moyenne entre celle de la plaque et celle de l'air à l'infini. Ceci équivaut à avoir un nombre de Rayleigh pour une plaque plane verticale de $1.1410^{9}$.

L'écoulement est donc laminaire à l'intérieur de la couche limite développée.

Le coefficient d'échange $\mathrm{h}_{0}$ résultant prend alors dans ces conditions la valeur :

$$
\mathrm{h}_{0}=2.7 \mathrm{~W} \mathrm{~m}^{-2} \mathrm{~K}^{-1}
$$

La valeur du coefficient d'échange calculée $\mathrm{h}_{0}$ est largement inférieure à la valeur $\mathrm{h}_{\text {app }}$ souhaitée $(1 / 10$ environ). Une autre solution autre que la plaque plane s'impose pour le dopage des échanges convectifs et qui prendra en considération les performances du capteur en période diurne.

La solution qui été retenue est celle d'un capteur muni d'ailettes rectangulaires sur sa partie basse, (fig. 1).L'échange se fera exclusivement par convection naturelle [5], Le problème consiste alors à bien dimensionner ces ailettes avec une forme, un écartement et une épaisseur optimale afin de dissiper la quantité voulue de chaleur.

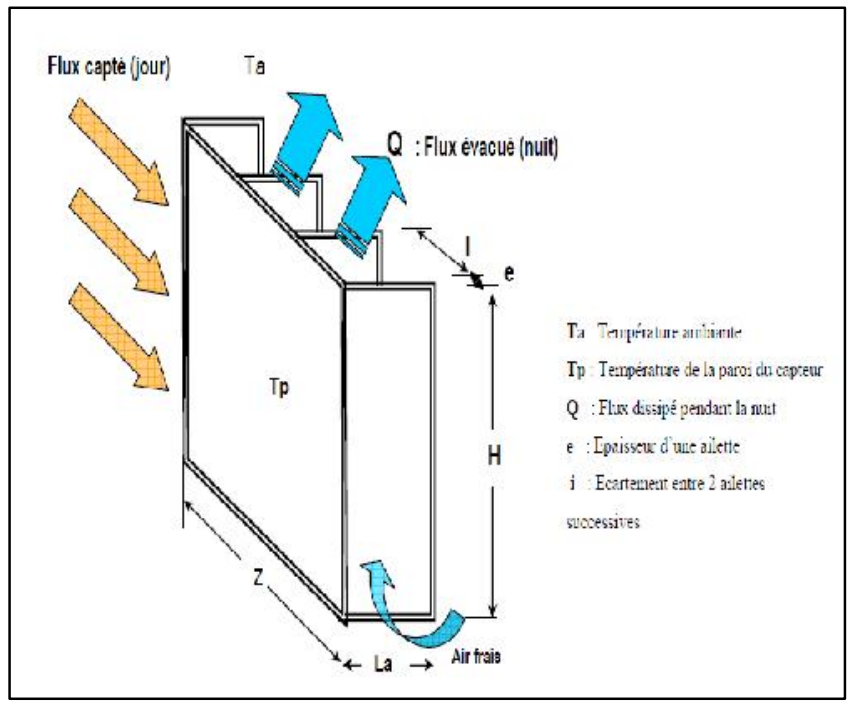

Figure 1 : Schéma du capteur régénérateur muni d'ailettes

\section{ETUDE THEORIQUE}

On suppose que la température est uniforme le long de la direction verticale de l'ailette. Le nombre total d'ailettes à implanter est $\mathrm{N}$ avec :

$$
\mathrm{N}-\frac{\mathrm{Z}}{\mathrm{e}+\mathrm{i}}
$$

Le coefficient d'échange apparent est :

$$
\mathrm{h}_{\mathrm{app}}=\frac{\mathrm{Q}}{\left[\mathrm{T}_{\mathrm{p}}-\mathrm{T}_{\mathrm{a}}\right] \mathrm{ZH}} \ldots \ldots \ldots \ldots \ldots
$$

Q étant le flux total dissipé :

Le coefficient d'échange apparent s'exprimera, d'après l'étude théorique [5], alors par

$$
h_{\text {app }}=\frac{z \cdot i \cdot H}{e+i} \frac{h}{z \cdot H}+\frac{2 N L a H h \eta}{z \cdot H} .
$$

Le coefficient réel d'échange h est calculé à partir de la corrélation : 


\begin{tabular}{|c|c|}
\hline & SEMINAIRE NATIONAL SUR LES SYSTEMES ENERGETIQUES HYBRIDES \\
$10-11$ Novembre 2015 \\
Université de Ghardaïa \\
Faculté des Sciences et de la Technologie
\end{tabular}

$$
N u_{i}=\frac{h . i}{\lambda}=\frac{1}{\sqrt{\frac{576}{\left(R a^{*}\right)^{2}}+\frac{2.873}{\left(R a^{*}\right)^{1 / 2}}}}
$$

Où $\mathrm{Ra}^{*}$ est le nombre de Rayleigh corrigé :

$$
R a^{*}=R a_{i} \frac{i}{H}
$$

De plus et pour faciliter les calculs, on définit les longueurs adimensionnelles de l'ailette :

Ecartement adimensionnel $x=\frac{i}{H} \sqrt[4]{R a_{H}}$

Epaisseur adimensionnelle $\quad e^{*}=\frac{\theta}{H} \sqrt[4]{R a_{H}}$

Longueur adimensionnelle $L a^{*}=\frac{L a}{H} \sqrt[4]{R a_{H}}$

L'équation (4) devient alors :

$$
\frac{h H}{\lambda_{a}}=\frac{x \sqrt[4]{R a_{H}}}{\sqrt{\left(\frac{576}{x^{4}}+2.873 x^{2}\right)}}
$$

Donc le coefficient d'échange apparent de la plaque sera :

$$
\frac{H h_{a p p}}{\lambda_{a}}=\frac{2 L a^{*} \sqrt[4]{R a_{H}}}{\left(1+\frac{e^{*}}{x}\right) \sqrt{\left(\frac{576}{x^{4}}+2.873 x^{2}\right)}}\left(\eta+\frac{x}{2 L a^{*}}\right)
$$

L'équation de la chaleur au sein de l'ailette s'écrit de la manière suivante [6] :

$$
\begin{aligned}
& \frac{d^{2} \theta}{d x^{2}}-m^{2} \theta=0 \ldots \ldots \ldots \ldots(10) \\
& \theta=T-T_{a} \quad T_{a} \text { étant la température de l'air. }
\end{aligned}
$$

Cette équation admet pour solution :

$$
\theta=\theta_{0} \frac{\cosh (m(L a-x))}{\cosh (m L a)}
$$

$$
\theta_{0}=T_{p}-T_{a}
$$

L'efficacité de l'ailette $\eta$, est définie par [5] :

$$
\eta=\frac{1}{L a} \frac{\int_{0}^{L a}\left(T-T_{a}\right) d L}{\left(T_{p}-T_{a}\right)}
$$

Soit

$$
\eta=\frac{-\left.\lambda H e \frac{d \theta}{d x}\right|_{x=0}}{2 \operatorname{LaHh}\left(T_{p}-T_{a}\right)}=\frac{\tanh (m L A)}{\mathrm{mLa}} .
$$

Avec : $m \cong \sqrt{\frac{2 h}{\lambda_{s}}}$ et $\lambda$ est conductivité thermique de l'ailette.

\section{4- RESULTATS}

Le coefficient d'échange est donné par l'équation (8).

Le gain correspond à l'implantation des ailettes est donc donné par :

$$
\frac{H h_{a p p}}{h_{0}}=\frac{2 L a^{4} \sqrt{2.873}}{\left(1+\frac{e^{*}}{x}\right) \sqrt{\left(\frac{576}{x^{4}}+2.873 x^{2}\right)}}\left(\eta+\frac{x}{2 L a^{*}}\right)
$$

A partir de cette équation, nous allons évaluer le gain apporté par les ailettes en fonction des caractéristiques géométriques de celles-ci.

\section{- Influence de l'épaisseur de l'ailette}

On remarque que l'augmentation de l'épaisseur de l'ailette engendre une légère diminution de la valeur du gain. Cependant cette influence est relativement faible. On choisi donc la valeur moyenne. On prendra comme valeur optimale $\mathrm{e}^{*}=0.50$ ce qui correspond à une épaisseur effective de l'ailette de $3 \mathrm{~mm}$. 


\begin{tabular}{|c|c|}
\hline & SEMINAIRE NATIONAL SUR LES SYSTEMES ENERGETIQUES HYBRIDES \\
$10-11$ Novembre 2015 \\
Université de Ghardaïa \\
Faculté des Sciences et de la Technologie
\end{tabular}

\section{- Influence de l'écartement}

Le gain augmente avec l'écartement et ceci jusqu'à une valeur optimale de $\mathrm{x}$ à déterminer puis décroît pour tendre vers 1 .

- Influence de la longueur de l'ailette

Pour déterminer la valeur optimale de l'écartement, on a représenté le gain en fonction de $\mathrm{La}^{*}$ pour différentes valeurs de x. On voit clairement que la valeur optimale est de 3 , ce qui correspond une distance entre deux ailettes successives de $1.6 \mathrm{~cm}$.

On remarquera en plus, que l'évolution du gain est presque linéaire, ce qui signifie que l'ailette transmet de la chaleur par convection sur la totalité sur cette longueur.

Cela correspond à une valeur réelle de la longueur de l'ailette : La<22

Enfin on constate que pour $\mathrm{x}=3$, le gain atteint une valeur de 10 , ceci pour une valeur de $\mathrm{La}^{*}=20$, soit une valeur réelle de l'ailette de $10 \mathrm{~cm}$.

\section{4- CONCLUSION ET DISCUSSIONS}

Le but de cette étude était de rechercher les dimensions géométriques optimales des ailettes à implanter dans le capteur régénérateur pour évacuer la quantité de chaleur voulue pendant la phase nocturne.

En conclusion à cette étude, on peut dire que la meilleure configuration géométrique pour l'obtention d'un coefficient d'échange apparent de l'ordre de $25 \mathrm{Wm}^{-2} \mathrm{k}^{-1}$ est réalisée avec les données suivantes : Epaisseur de l'ailette e $=4 \mathrm{~mm}$, écartement $\mathrm{i}=1.6 \mathrm{~cm}$ et une longueur de l'ailette $\mathrm{de} \mathrm{La}=10 \mathrm{~cm}$. Ces paramètres apparaissent encouragent du point de vu réalisation.

\section{5- REFERENCES BIBLIOGRAPHIQUES}

[1] Hilbrand C., Dind P., Pons M., Buchter F., "A new solar powered adsorption refrigerator with high performance", Solar Energy, 77, 311-318, 2004.

[2]- Nolwenn Le Pierres, «Procédé solaire de production de froid base température $\left(-28^{\circ} \mathrm{C}\right.$ par sorption solide-gaz », thèse soutenue en septembre 2005, Université de Perpignan France

[3] N Hebbir "dopage des échanges par convection naturelle en cycle nocturne" mémoire de magister setenue en juin 2006. Université Larbi ben M'hidi Oum el bouagui. Algérie.

[4] CNRS, rapport final d'activité, GAT 6 : Habitat, « Congélation solaire $\left(-30^{\circ} \mathrm{C}\right)$ par procédé thermochimique à partir d'une source thermique basse température $\left(70^{\circ} \mathrm{C}\right) »$, (2004).

[5] Andréa sz lieto vollaro, stefano grignaffini, franco gugliermitti "Optimum design of vertical rectangular fin arrays" Int. Jour. Sc. 38. pp 525 -529 (1999).

[6] André B. De Vrient, « La transmission de la chaleur », Ed. Gaëtan Morin, 1984. 


\begin{tabular}{|c|c|} 
SEMINAIRE NATIONAL SUR LES SYSTEMES ENERGETIQUES HYBRIDES \\
$10-11$ Novembre 2015 \\
Université de Ghardaïa \\
Faculté des Sciences et de la Technologie
\end{tabular}
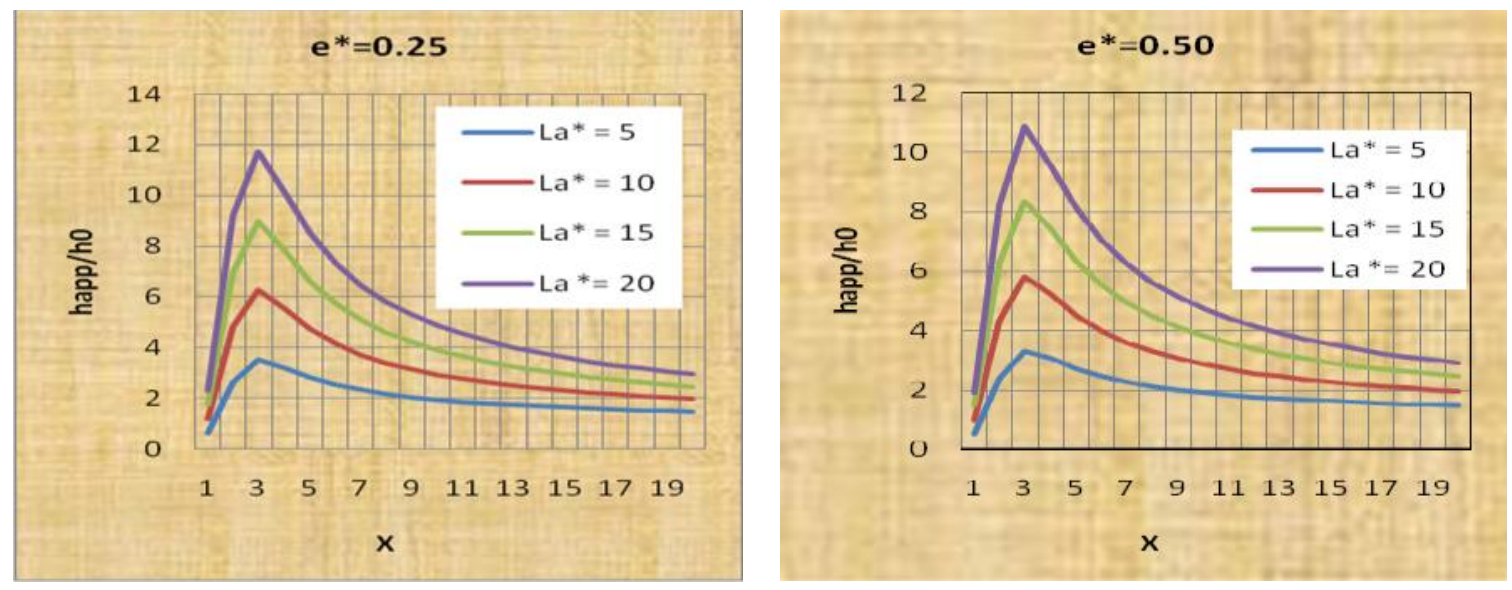

Figure 2 : Influence de $\mathrm{e}^{*}$ sur le gain en fonction de l'espacement $\mathrm{x}$
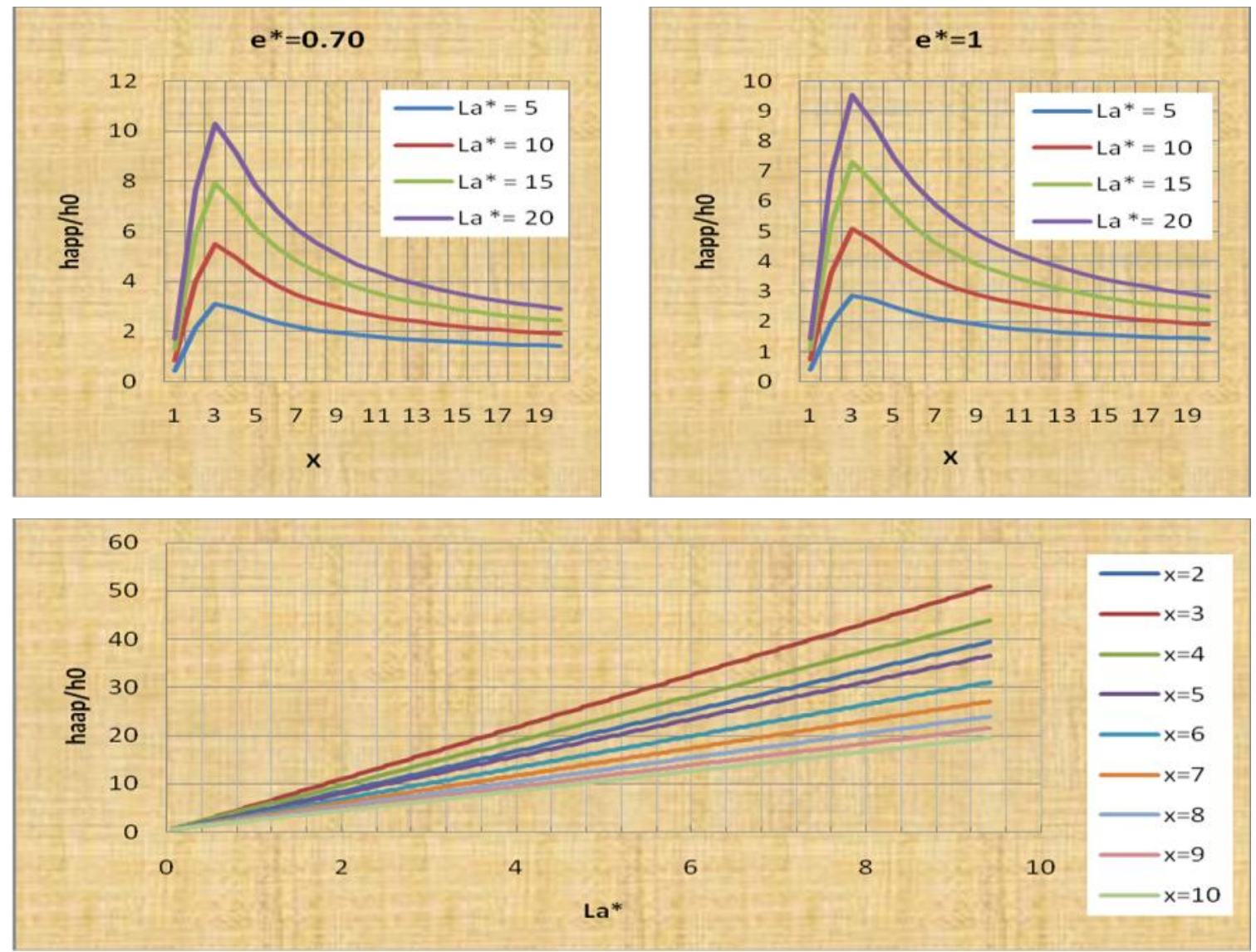

Figure 3 : Calcul du gain en fonction de La* pour différentes valeurs de $\mathrm{x}$. Cas de $\mathrm{e}^{*}=0.7$ 


\title{
Simulation et dopage des échanges par convection naturelle d'un capteur régénérateur d'une machine frigorifiques solaire. Cas d'ailette rectangulaire
}

\author{
N. Hebbir ${ }^{*}$ et N. Labed \\ Département de Mécanique, Centre Universitaire Larbi Ben M’Hidi, Oum El Bouaghi
}

\begin{abstract}
Résumé - Le besoin en froid dans le secteur de l'habitat individuel ou collectif est en forte augmentation, l'une des applications (qui répond aux exigences environnementales) est l'utilisation des machines frigorifiques à adsorption solaire. Celles-ci sont équipées par des capteurs régénérateurs. Ils ont comme rôle; de capter l'énergie solaire le jour (phase diurne), et évacuer la chaleur du réacteur en phase nocturne. Les premières évaluations du projet montrent que le problème se trouve au niveau de la phase de production de froid (nuit). La solution proposée est l'adjonction des ailettes rectangulaires sur la paroi du capteur. Notre objectif ici est de faire une étude par simulation de ce type d'ailette, afin de doper les échanges par convection naturelle en cycle nocturne utilisant le code de simulation Fluent.
\end{abstract}

Abstract - The need of cooling in the individual or collective housing sector is rising sharply, one of the applications (which gives the answer to environmental requirements) is the use of solar adsorption refrigeration machinery. They are equipped with regenerators sensors. Their role, is to capture solar energy during the day (diurnal phase), and remove heat from the reactor in the night period. The first evaluations of the project show that the problem lies in the production phase of cold (night).The proposed solution is the addition of rectangular fins on the side of the sensor. Our goal here is to make a simulation study of this type of wing, to boost exchange by natural convection at night cycle using the simulation code Fluent.

Mots clés: Machine frigorifique solaire - Convection naturelle - Capteur solaire - Ailettes rectangulaires - Simulation - Fluent.

\section{INTRODUCTION}

Avec l'accroissement de la population et le développement industriel, surtout dans les régions pétrolières, les besoins de l'Algérie en froid sont de plus en plus importants. Le recours aux énergies renouvelables, surtout solaire, devient plus que nécessaire pour répondre à ces besoins.

L'un des procédés de production de froid par énergie solaire est le procédé thermochimique à adsorption. Plusieurs laboratoires français sont impliqués pour la conception d'une nouvelle machine frigorifique solaire à adsorption. Elle devra réaliser une température de $\left(-30^{\circ} \mathrm{C}\right)$ et utilisera une source solaire basse température de $70^{\circ} \mathrm{C}$ contrairement au machines existantes actuelles.

Les premières évaluations $\mathrm{du}$ projet ont montré que les contraintes de dimensionnements les plus fortes se situaient non pas au niveau de captation de l'énergie solaire, mais au contraire, au niveau du refroidissement du réacteur dans la phase nocturne de production.

*nacer_habb@yahoo.fr_nabil_lab@yahoo.fr 


\section{PROBLEMATIQUE}

L'objectif de cette étude, est de concevoir un capteur performant le jour, et possédant une fonction de radiateur, durant la phase nocturne de régénération, le flux à évacuer est de $250 \mathrm{~W} / \mathrm{m}^{2}$ à travers une surface de $10 \mathrm{~m}^{2}$, soit un coefficient d'échange apparent de l'ordre de $\mathrm{h}_{\text {app }}=1 \mathrm{Wm}^{-2} \mathrm{~K}^{-1}$. Une étude préliminaire d'optimisation a déjà permis de fixer les dimensions des ailettes à implanter sur la partie basse du capteur [3].

L'objectif de ce travail est donc, de simuler pour essayer de doper les échanges thermiques par convection naturelle au niveau des ailettes. Il faut notamment vérifier que le capteur choisi est tout à fait capable d'évacuer la quantité de chaleur donnée pendant le temps fixé. Nous avons opté pour le code 'Fluent' largement utilisé dans le domaine de la mécanique des fluides et de la thermique.

\section{DOMAINE D'ETUDE}

Le capteur aileté par sa face inférieure présente une périodicité régulière. On dispose donc de deux plans de symétrie. Le domaine d'étude sera réduit à un motif élémentaire englobant une demi ailette et un demi écartement. Seule, la dernière ailette aura un traitement particulier. Les conditions aux limites sont à déterminer.

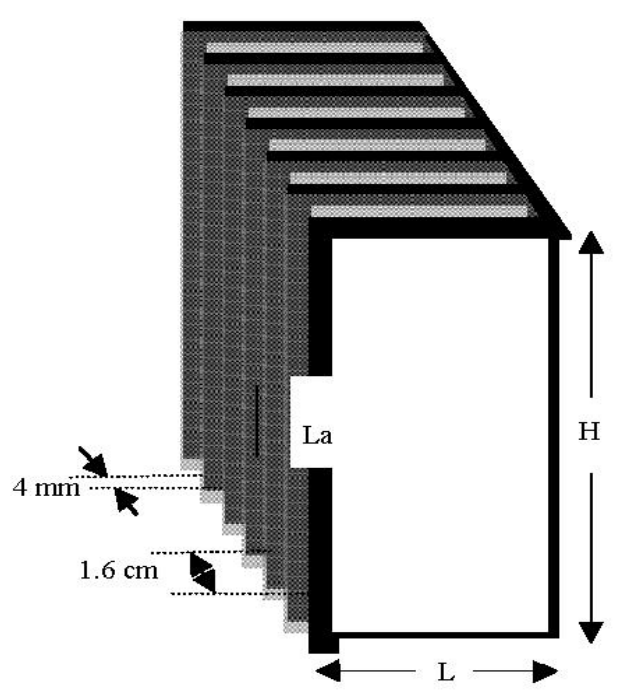

Fig. 1: Vue en perspective du capteur muni d'ailettes

\subsection{Conditions aux limites}

Avec le lexique du code, nous avons:

3.1.1 Les faces avant et arrière sont caractérisées par une condition de symétrie 'symmetry' (symétrie).

3.1.2 La paroi du capteur est associée à une condition de température imposée $\mathrm{T}_{\mathrm{p}}=10^{\circ} \mathrm{C}$. 
3.1.3 La conduction dans l'ailette est prise en compte, autrement dit celle-ci est considéré comme élément solide "solid", de conductivité thermique $\lambda=204.4 \mathrm{Wm}^{-1} \mathrm{~K}^{-1}$, ce qui correspond à la conductivité thermique de l'aluminium.

Les frontières ouvertes du domaine sont caractérisées par les conditions aux limites:

\subsubsection{Vitesse d'entrée}

Cette condition permet d'imposer sur une surface une vitesse et une température du fluide entrant. La vitesse est estimée à $0.03 \mathrm{~m} / \mathrm{s}$.

\subsubsection{Débit fluide sortant}

Cette condition est traduite le fait qu'on ne connaît pas les paramètres (vitesse et pression) du fluide sortant.

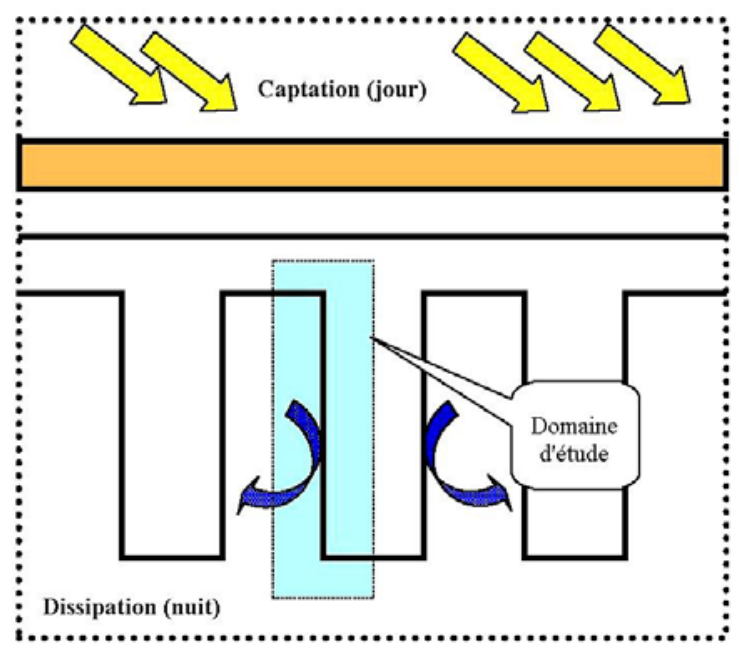

Fig. 2: Vue de dessus du domaine d'étude

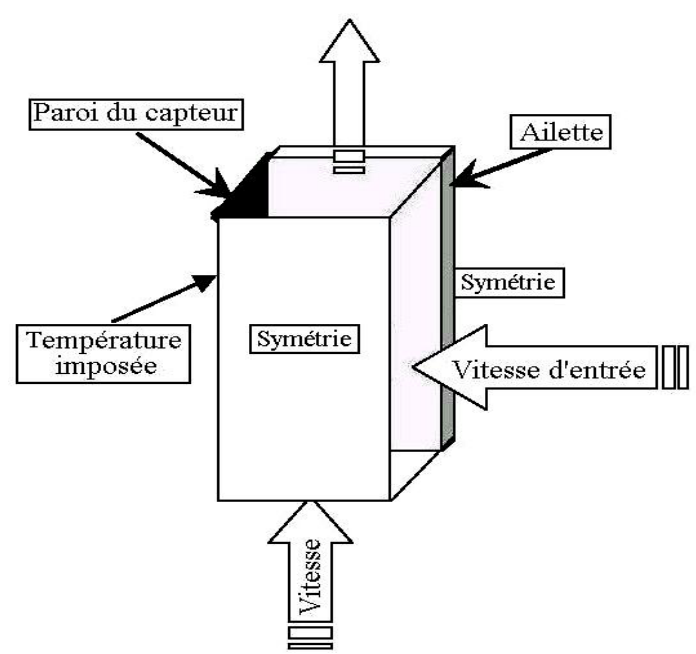

Fig. 3: Condition aux limites 


\section{MAILLAGE}

L'écoulement de l'air autour des ailettes en convection naturelle est supposé laminaire en régime stationnaire, selon un modèle isolé en formulation implicite. Le fluide est supposé incompressible sous l'hypothèse de Boussinesq.

Etant donné que le domaine d'étude ne présente pas de singularité particulière, ce maillage est généré grâce au logiciel 'GAMBIT'. Les pas d'espace suivant, $\mathrm{x}, \mathrm{y}, \mathrm{z}$ sont respectivement égaux à $4 \mathrm{~mm}, 50 \mathrm{~mm}, 1 \mathrm{~mm}$.

Rappelons que la problématique consiste à bien dimensionner les ailettes du capteur pour dégager la quantité de chaleur fixée pendant un temps limité. Pour la présentation des résultats obtenus, nous avons choisi de représenter les isothermes et les profils de vitesses.

On s'est intéressé aux ailettes rectangulaires afin de nous permettre de comparer nos résultats avec ceux du LET [1]. Ces ailettes, de conception simple, peuvent être tout a fait acceptables au niveau des échange thermique.

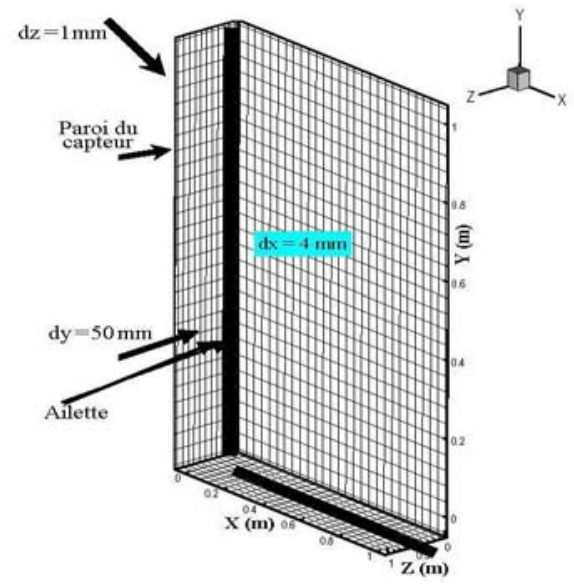

Fig. 4: Maillage du domaine

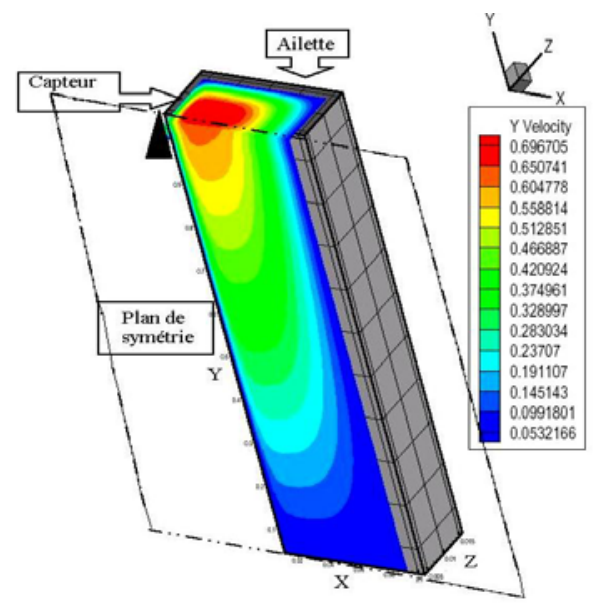

Fig. 5: Profils 3D de vitesses de l'air

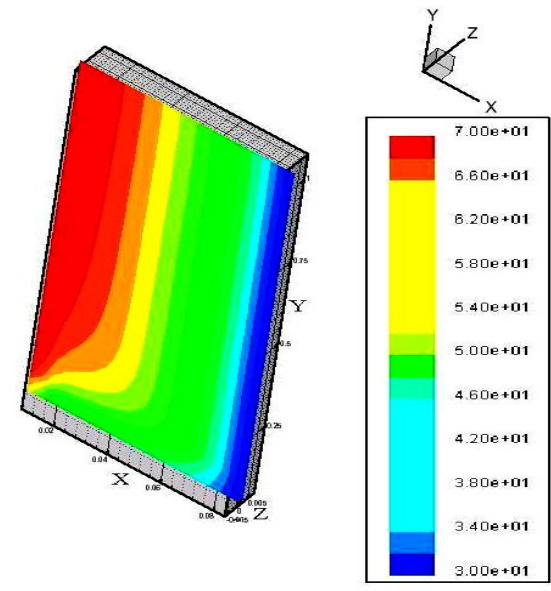

Fig. 6: Profils de températures sur le plan de symétrie 
Les profils de vitesses sur la figure 5 sont calculés pour une longueur de l'ailette égale à $8 \mathrm{~cm}$ pour une épaisseur de $4 \mathrm{~mm}$. On peut observer l'évolution du profil de vitesse de l'air le long de l'ailette. A la base du capteur, la vitesse de l'air est nulle. Mais au contact de l'ailette, l'air s'échauffe et une force ascensionnelle est créée permettant à l'air d'accélérer son mouvement vers le haut. Le coefficient d'échange est d'autant plus fort que la vitesse de l'air est plus importante.

On a considéré que la vitesse de l'air à l'entrée (coté bas et avant) est nulle. A la sortie du domaine, la vitesse atteint son maximum avec une valeur importante (environ $0.70 \mathrm{~m} / \mathrm{s}$ ). Ce qui traduit un échauffement important et donc une bonne convection.

Sur les mêmes figures, sont représentés les profils de vitesse entre deux ailettes successives. On voit que la majorité des échanges est assurée par la moitié des ailettes, l'interaction entre les ailettes y est claire.

Sur les figures 6, 7 et 8 , on propose les profils de températures sur les plans de symétries avant et le plan perpendiculaire par rapport à l'ailette. On voit clairement le développement de la couche limite de convection naturelle le long de la paroi du capteur, ce qui traduit bien l'effet de refroidissement du capteur. On remarque aussi que la majorité des échanges est assuré par la moitié de l'ailette.

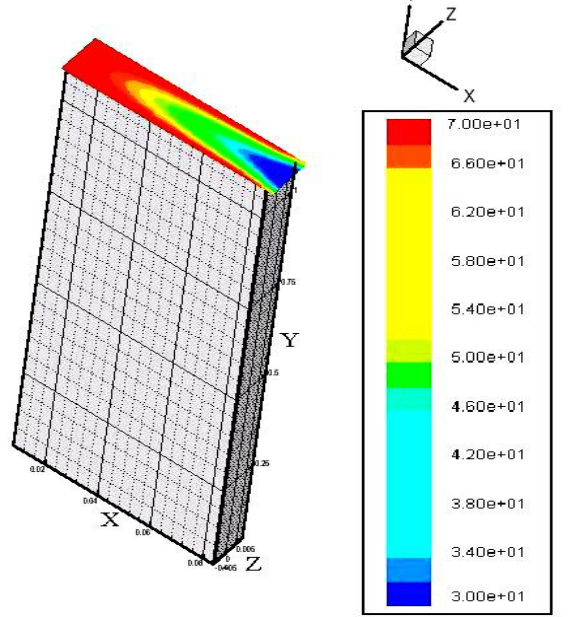

Fig. 7: Profils de températures entre 2 ailettes successives plan à $1 \mathrm{~m}$ de hauteur

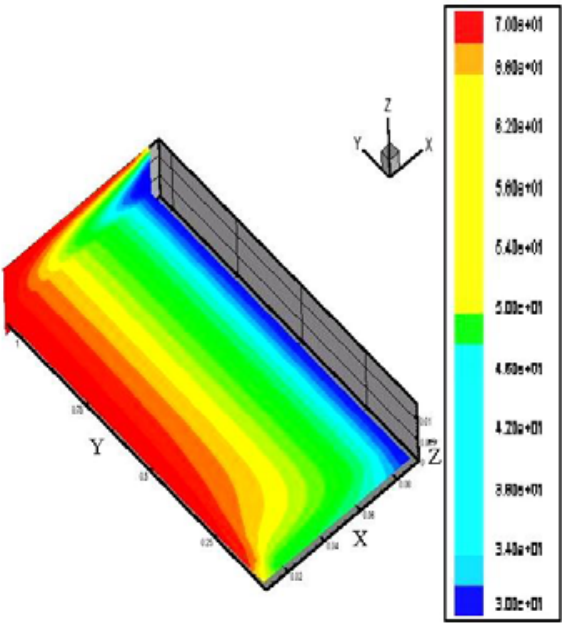

Fig. 8: Profils de températures entre 2 ailettes successives en $3 \mathrm{D}$

A la lecture des résultats obtenus, on peut dire qu'il est tout à fait possible de réaliser, avec des ailettes rectangulaires, l'échange thermique souhaité par convection naturelle uniquement pendant le temps fixé. Ceci rejoint les conclusions de l'étude préliminaire effectuée au LET [1] même si, pour notre calcul, on a considéré les conditions climatiques algériennes.

\section{SENSIBILITE AU MAILLAGE}

Afin de confirmer les résultats trouvés, nous avons effectué un changement de paramètres de maillage.

L'étude de sensibilité au maillage a été fait par le jeu des paramètres suivants: $\mathrm{i}=16 \mathrm{~cm}, \mathrm{e}=4 \mathrm{~mm}$ et $\mathrm{L}_{\mathrm{a}}=8 \mathrm{~cm}$. 
La figure 9 représente l'influence de l'affinage du maillage sur le profil de température et la composante verticale de la vitesse dans le plan de symétrie avant à mihauteur du capteur.

La courbe noire est celle du maillage de base alors que celle de couleur violet est celle obtenue avec le maillage fin (la moitié du pas) La jaune est celle du maillage large (le double du pas de base).

On voit clairement que les résultats trouvés ne présentent qu'une légère sensibilité au maillage, on peut donc conclure que les résultats de la simulation sont acceptables.

\section{CONCLUSION}

En conclusion à cette étude, nous pouvons dire que le choix d'ailette rectangulaire simple est tout à fait satisfaisant. Le flux à évacuer pour permettre la régénération du fluide frigorigène est suffisamment dissipé par la partie basse du capteur-régénérateur.

En plus, nos résultats sont comparables avec ceux effectué au LET [1], malgré que nous avons entièrement refait l'étude. Une étude comparative avec d'autres types d'ailettes permettrait certainement de rendre notre étude plus complète.

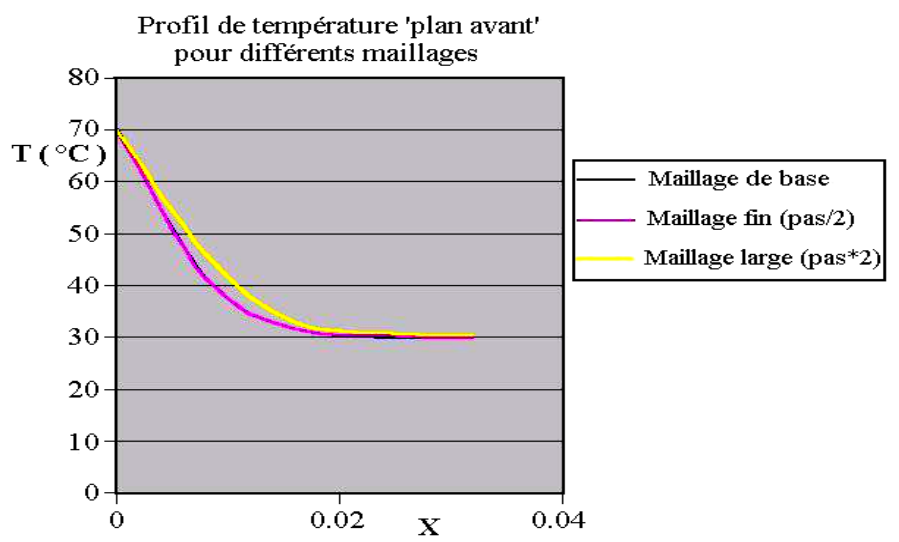

Fig. 9: Sensibilité au maillage pour le calcul de la température

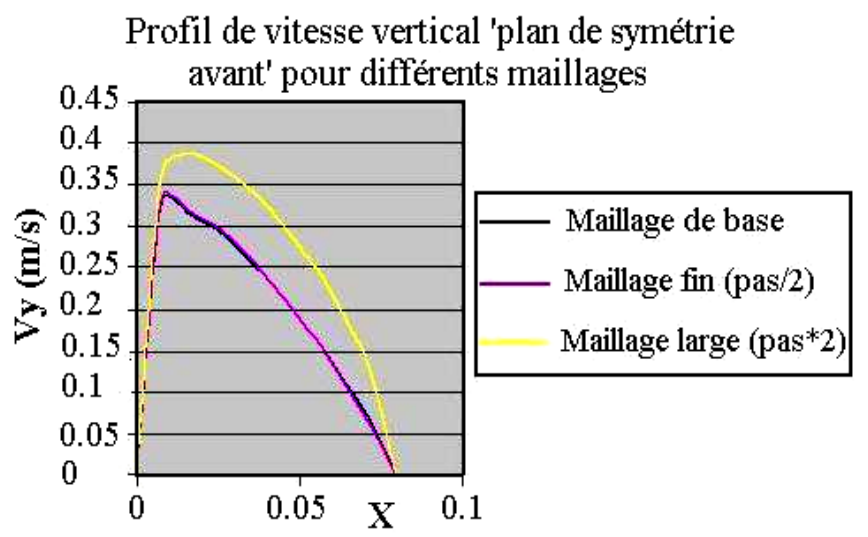

Fig. 10: Sensibilité au maillage pour le calcul des vitesses 


\section{REFERENCES}

[1] LET. Rapport de Projet PRI 6.1, 'Froid Solaire - Dopage des Echanges par Convection Naturelle', Poitiers, 2004.

[2] A. sz lieto Vollaro, S. Grignaffini, F. Gugliermitti, 'Optimum Design of Vertical Rectangular Fin Arrays', Int. J. Sc., Vol. 38, pp. 525 - 529, 1999.

[3] N. Hebbir et N. Labed, 'Optimisation des Echanges Thermiques par Convection Naturelle d'un Capteur Régénérateur d'une Machine Frigorifique Solaire', Séminaire International sur la Physique Energétique, SIPE'8, Béchar, Algérie, Novembre 2006.

[4] N. Le Pierres, D. Stitou et N. Mazet, 'Conception d'un Procédé Thermochimique de Congélation Solaire à Partir de la Chaleur Basse Température', Institut des Sciences et Génie des Matériaux et Procédés, 'IMP', UPR CNRS 8521, Rambla de la Thermodynamique, Tecnosud, Perpignan, France.

[5] N. Le Pierres, 'Procédé Solaire de Production de Froid Basse Température $-28{ }^{\circ} \mathrm{C}$ par Sorption Solide - Gaz', Thèse de Doctorat, Université de Perpignan, septembre 2005.

[6] A.B. De Vrient, 'La Transmission de la Chaleur', Edition Gaëtan Morin, 1984.

[7] CNRS, Rapport Final d'Activité 2002/2004, GAT 6: Habitat, 'Congélation Solaire $-30{ }^{\circ} \mathrm{C}$ par un Procédé Thermochimique à partir d'une Source Thermique Basse Température $\left(70^{\circ} \mathrm{C}\right)$ ', 2002-2004. 


\section{Résumé :}

L'utilisation des énergies renouvelables comme source de chaleur montre un double avantage : limiter la pollution et réduire le coût du combustible.

Dans les dernières décennies, la demande du froid est en forte augmentation. Surtout dans les régions dépourvues du réseau électrique.

L'une des applications est l'utilisation des machines frigorifiques à adsorption solaire. Elles utilisent le couple gel de silice-eau, c'est dans cette perspective que s'inscrit ce travail, dans l'objectif est de réaliser et expérimenter un prototype à fonctionnement cyclique.

Les résultats expérimentaux trouvés sont satisfaisante et peuvent construire une plateforme de recherche dans notre payé, dont le but est d'améliorer le rendement de l'installation expérimentale d'une part, et de maitriser ce procédé de production de froid, mal exploité en Algérie, d'autre part.

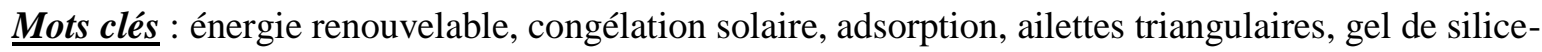
eau.

\section{$\underline{\text { Abstract : }}$}

The use of renewable energy as a heat source shows a double advantage: reduce pollution and reduce the cost of fuel.

In recent decades, the application of cold is rising sharply. Especially in regions without electricity supply.

One application is the use of solar adsorption chillers. The use of silica gel water is the task which fits the objective to realize and experience a cyclically operating prototype.

The experimental results are found satisfactory and can build a research platform in our country, which aims to improve the performance of the test facility on one hand, and to master the cold sore production process operated in Algeria.

Keywords: renewable energy, solar freezing, adsorption, triangular fins, water-silica gel

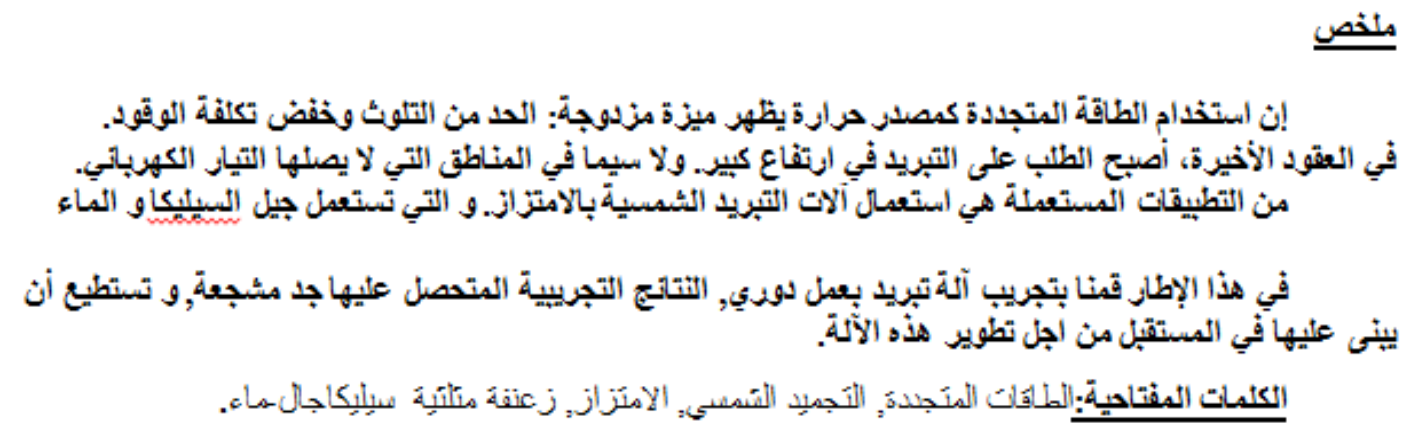

